\title{
Turbulence, Magnetic Reconnection in Turbulent Fluids and Energetic Particle Acceleration
}

\author{
A. Lazarian · L. Vlahos · G. Kowal · H. Yan · A. Beresnyak · E. M. de Gouveia Dal \\ Pino
}

Received: date / Accepted: date

\begin{abstract}
Turbulence is ubiquitous in astrophysics. It radically changes many astrophysical phenomena, in particular, the propagation and acceleration of cosmic rays. We present the modern understanding of compressible magnetohydrodynamic (MHD) turbulence, in particular its decomposition into Alfvén, slow and fast modes, discuss the density structure of turbulent subsonic and supersonic media, as well as other relevant regimes of astrophysical turbulence. All this information is essential for understanding the energetic par-
\end{abstract}

A. Lazarian

Department of Astronomy, University of Wisconsin-Madison, $475 \mathrm{~N}$. Charter St., Madison, WI, 53706, USA

Tel.: +1-608-262-1715

Fax: +1-608-263-6386

E-mail: lazarian@astro.wisc.edu

L. Vlahos

Department of Physics, University of Thessaloniki, 54124 Thessaloniki, Greece

E-mail: vlahos@astro.auth.gr

G. Kowal

Instituto de Astronomia, Geofísica e Ciências Atmosféricas, Universidade de São Paulo, Rua do Matão, 1226 - Cidade Universitária, CEP 05508-090, São Paulo/SP, Brazil

E-mail: kowal@astro.iag.usp.br

H. Yan

Kavli Institute, Peking University, 5 Yi He Yaun Rd, Bejing, 100871

China

E-mail: hryan@pku.edu.cn

A. Beresnyak

Los Alamos Laboratory, Los Alamos, NM, 87545, USA

and

Ruhr-Universitat University, Bochum, 44789, Bochum, Germany

E-mail: beresnyak@wisc.edu

E. M. de Gouveia Dal Pino

Instituto de Astronomia, Geofísica e Ciências Atmosféricas, Universidade de São Paulo, Rua do Matão, 1226 - Cidade Universitária, CEP 05508-090, São Paulo/SP, Brazil

E-mail: dalpino@astro.iag.usp.br ticle acceleration that we discuss further in the review. For instance, we show how fast and slow modes accelerate energetic particles through the second order Fermi acceleration, while density fluctuations generate magnetic fields in pre-shock regions enabling the first order Fermi acceleration of high energy cosmic rays. Very importantly, however, the first order Fermi cosmic ray acceleration is also possible in sites of magnetic reconnection. In the presence of turbulence this reconnection gets fast and we present numerical evidence supporting the predictions of the Lazarian \& Vishniac (1999) model of fast reconnection. The efficiency of this process suggests that magnetic reconnection can release substantial amounts of energy in short periods of time. As the particle tracing numerical simulations show that the particles can be efficiently accelerated during the reconnection, we argue that the process of magnetic reconnection may be much more important for particle acceleration than it is currently accepted. In particular, we discuss the acceleration arising from reconnection as a possible origin of the anomalous cosmic rays measured by Voyagers as well as the origin cosmic ray excess in the direction of Heliotail.

Keywords Turbulence $\cdot$ Magnetic reconnection - Acceleration $\cdot$ Cosmic rays

\section{Introduction}

It is well known that astrophysical fluids are magnetized and turbulent (see Armstrong and Woo 1981; Armstrong et al. 1995; Verdini and Velli 2007; Lazarian 2009, and references therein). For interstellar medium Fig. 11illustrates the turbulent power density plotted against the inverse of the scale length, with data at large scales, i.e. at small wave numbers $q$ expanded using the Wisconsin $\mathrm{H}_{\alpha}$ Mapper (WHAM) data 


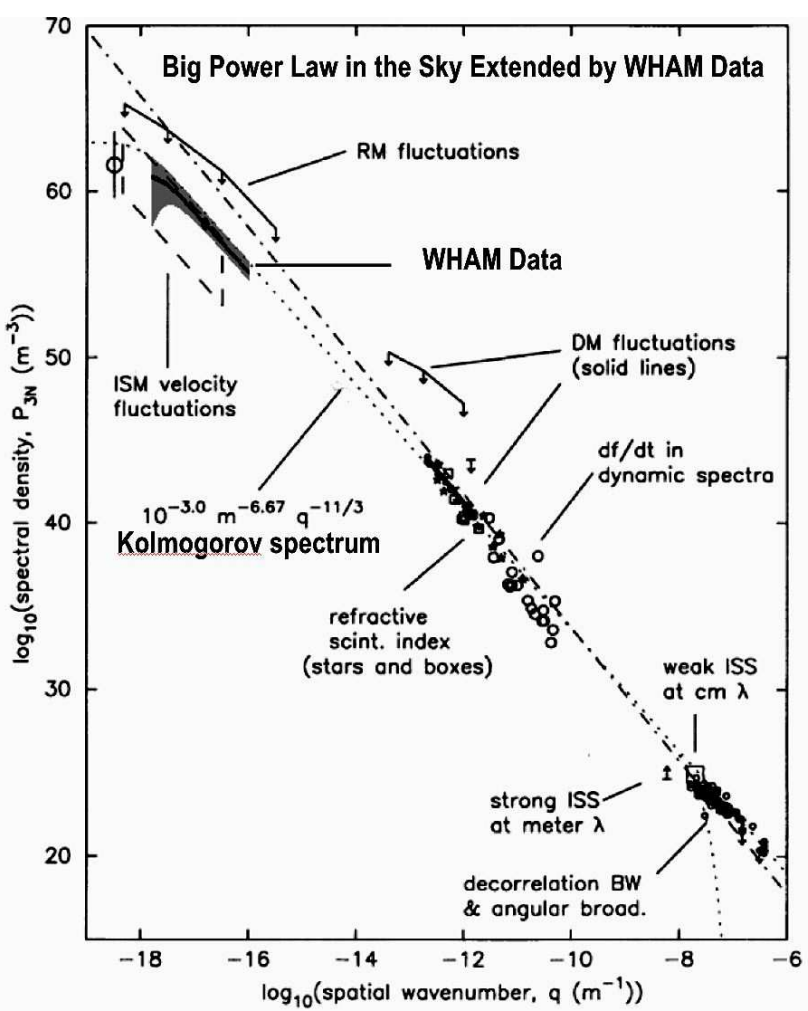

Fig. 1 Turbulence in the interstellar gas as revealed by electron density fluctuations. "Big Power Law in the Sky" in Armstrong et al. (1995) extended using WHAM data. The slope corresponds to that of Kolmogorov turbulence. Modified from Chepurnov and Lazarian (2010).

on electron density fluctuations 1 (Chepurnov and Lazarian 2010). A similar Kolmogorov-type power law is measured with in situ measurements in Solar wind Coleman (1968); Matthaeus et al. (1982); Matthaeus and Goldstein (1982); Leam (1998) On much larger scales, clusters of galaxies also reveal turbulent spectrum (Enßlin and Vogt 2006).

The fact that turbulence is ubiquitous in astrophysical environments is the most natural and expected. Magnetized astrophysical plasmas generally have very large Reynolds numbers due to the large length scales involved, as well as the fact that the motions of charged particles in the direction perpendicular to magnetic fields are constrained. Laminar plasma flows at these high Reynolds numbers $R=V L_{f} / v$, where $V$ and $L_{f}$ are the velocity and the scale of the flow, $v$ is fluid viscosity, are prey to numerous linear and finite-

1 A more direct evidence comes from the observations of spectral lines. Apart from showing non-thermal Doppler broadening (see Larson 1981), they also reveal spectra of supersonic turbulent velocity fluctuations when analyzed with techniques like Velocity Channel Analysis (VCA) of Velocity Coordinate Spectrum (VCS) developed (see Lazarian and Pogosyan 2000, 2004, 2006, 2008) and applied to the observational data (see Padoan et al. 2004, 2009; Chepurnov et al. 2010).

2 More discussion of the solar wind turbulence can be found in Bruno and Carbone (2005); Petrosyan et al. (2010) amplitude instabilities, from which turbulent motions readily develop.

For interstellar medium the drivers of turbulence include supernovae explosions that shape the interstellar medium (McKee and Ostriker 1977; Nakamura et al.2006), accretion flows (Klessen and Hennebelle 2010), magneto-rotational instability in the galactic disk (Sellwood and Balbus 1999), thermal instability (see Kritsuk and Norman 2002; Kovama and i. Inutsuka 2002), collimated outflows (see Nakamura and Li 2007), etc.. Cluster mergers, jets of radio galaxies and turbulent galaxy wakes are among the drivers of turbulence in clusters of galaxies (Brunetti et al.2009). Similarly, the fast plasma flow and plasma instabilities provide the natural environment for turbulence to develop in the solar wind. In more special circumstances, instabilities in the fluid of cosmic rays as well as energy injection via magnetic reconnection can serve as the source of turbulence. The latter provide special cases that we also consider in the paper, but the generic set up is that both the evolution of cosmic rays and reconnection is considered in the presence of pre-existing turbulence which is created by external sources 3 .

The model that has been shown to successfully reproduce the properties of incompressible MHD turbulence was proposed by Goldreich and Sridhar (1995, henceforth GS95). Numerical studies of MHD turbulence in Cho and Lazarian (2002, 2003) allowed to extend the latter model to compressible fluids. This allowed to change our understanding of the effects of turbulence on the scattering and acceleration of cosmic rays (Yan and Lazarian 2002, 2004).

Turbulence is known to affect most of the properties of fluids, e.g. thermal conductivity, propagation of waves and energelic particles, magnetic field generation etc. (see reV1ews and books by Moffatt 1978; Dmitruk et al. 2001; Schlickeiser 2003; Vishniac et al. 2003; Cranmer and van Ballegooijen 2005; Longair 2010, and references therein). For instance, the cosmic ray streaming instability that is the basic textbook process is being suppressed in the presence of turbulence in the interstellar medium (Yan and Lazarian 2002; Farmer and Goldreich 2004; Beresnyak and Lazarian 2008). Similarly, Lazarian and Vishniac (1999, henceforth LV99) shows that the constrains on the classical Sweet-Parker reconnection are being lifted in the presence of turbulence and the reconnection rate becomes fast, i.e. independent on resistivity.

Fast magnetic reconnection predicted in the presence of turbulence in Lazarian and Vishniac (1999) entails many astrophysical consequences (see e.g. Lazarian 2011, for the consequences for star formation). It entails, for instance, the efficient acceleration of cosmic rays in reconnection regions as discussed in de Gouveia Dal Pino and Lazarian (2005) (see also Lazarian 2005).

\footnotetext{
3 The relevant analogy: turbulence that is experienced by the passengers of an airplane does not arise from the airplane motion, but originates due to complex processes taking place in the atmosphere.
} 
In what follows, we discuss the nature of MHD turbulence in $\S 2$, its consequences for magnetic reconnection in $\S 3$ and for cosmic ray acceleration in $\S 4$. We describe the first order Fermi acceleration that takes place in the reconnection sites in $\S 5$. The discussion and summary are presented in $\S 6$ and $\S 7$, respectively.

\section{MHD turbulence in astrophysical plasmas}

MHD turbulence is widely spread process that is essential both for astrophysical reconnection and cosmic ray physics. The balanced MHD turbulence is relatively well explored subject, but other regimes of MHD turbulence, e.g. imbalanced MHD turbulence, viscosity dominated MHD turbulence are still insufficiently studied and the consequences of these regimes are poorly explored.

\subsection{Applicability of MHD approximation}

The paper is focused on the properties of MHD turbulence as we claim that the small scale plasma effects are of secondary importance for both the dynamics of turbulence at sufficiently large scales as well as for reconnection and acceleration of cosmic rays in the presence of turbulence.

The applicability of MHD description to plasmas has been revisited recently in Eyink et al. (2011, henceforth ELV11). There three characteristic length-scales were considered: the ion gyroradius $\rho_{i}$, the ion mean-free-path length $\ell_{m f p, i}$, and the scale $L$ of large-scale variation of magnetic and velocity fields. Astrophysical plasmas are in many cases "strongly collisional" in the sense that $\ell_{m f p, i} \ll \rho_{i}$, which is the case for the interiors of stars and accretion disks. In such cases, a fluid description of the plasma is valid. In the "weakly collisional" $\ell_{m f p, i} \gg \rho_{i}$, The ratio

$\frac{\ell_{m f p, i}}{\rho_{i}} \propto \frac{\Lambda}{\ln \Lambda} \frac{v_{A}}{c}$,

follows from the standard formula for the Coulomb collision frequency (see Fitzpatrick 2008, Eq. 1.25). Here $\Lambda=4 \pi n \lambda_{D}^{3}$ is the plasma parameter, or the number of particles within the Debye screening sphere. When astrophysical plasmas are very weakly coupled (hot and rarefied), then $\Lambda$ is large, e.g. of the order of $10^{9}$ or more for the warm component of the interstellar medium or solar wind (see Table 1). For such ratio the expansion over small ion Larmor radius $\rho_{i}$ provides "kinetic MHD equations" which differ from the standard MHD by having anisotropic pressure tensor.

Plasmas that are not strongly collisional further subdivided into two classes: "collisionless" plasmas for which $\ell_{m f p, i} \gg L$, the largest scales of interest, and "weakly collisional" plasmas for which $L \gg \ell_{m f p, i}$. In the latter case the "kinetic MHD" description can be further reduced in complexity at scales greater than $\ell_{m f p, i}$ (see ELV11). This reproduces a fully hydrodynamic MHD description at those scales, with anisotropic transport behavior associated to the well-magnetized limit. Among our examples in Table 1 above, the warm ionized ISM is "weakly collisional", while postCME current sheets and the solar wind impinging on the magnetosphere are close to being "collisionless."

Additional important simplifications occur if the following assumptions are satisfied: turbulent fluctuations are small compared to the mean magnetic field, have length-scales parallel to the mean field much larger than perpendicular length-scales, and have frequencies low compared to the ion cyclotron frequency. These are standard assumptions of the Goldreich and Sridhar (1995) theory of MHD turbulence. They are the basis of the "gyrokinetic approximation" (Schekochihin et al. 2007, 2009). At length-scales larger than the Larmor radius $\rho_{i}$, another reduction takes place. The incompressible shear-Alfvén wave modes exhibit dynamics independent of compressive motions and can be described by the "Reduced MHD” (RMHD) equations (see Strauss 1976; Zank and Matthaeus 1992a; Goldreich and Sridhar 1995; Cho and Lazarian 2003).

This fact is essential for the LV99 justifying the use of the treatment based on an incompressible MHD model.

\subsection{MHD turbulence: cascades of Alfénic, slow and fast} modes

Magnetized turbulence is a tough and complex problem with many excellent monographs and reviews devoted to different aspect of it (see Biskamp 2003, and references therein). A broad outlook on the astrophysical implications of the turbulence can be found in a review by Elmegreen and Scalo (2004), while the effects of turbulence on molecular clouds and star formation are well reviewed in McKee and Ostriker (2007). However, the issues of turbulence spectrum and its anisotropies, we feel, are frequently given less attention than they deserve.

We deal with magnetohydrodynamic (MHD) turbulence which provides a correct fluid-type description of plasma turbulence at large scales. Astrophysical turbulence is a direct consequence of large scale fluid motions experiencing low friction. The Reynolds numbers are typically very large in astrophysical flows as the scales are large. As magnetic fields decrease the viscosity for the plasma motion perpendicular to their direction, Re numbers get really astronomically large. For instance, $R e$ numbers of $10^{10}$ and larger are very common for astrophysical flows. For so large $R e$ the inner degrees of fluid motion get excited and a complex pattern of motion develops.

While turbulence is an extremely complex chaotic nonlinear phenomenon, it allows for a remarkably simple statistical description (see Biskamp 2003). If the injections and 
Table 1 Parameters for Some Astrophysical Plasmas (adopted from Eyink et al. 2011).

\begin{tabular}{llll}
\hline \hline Parameter & $\begin{array}{l}\text { warm ionized } \\
\text { ISM }^{a}\end{array}$ & $\begin{array}{l}\text { post-CME } \\
\text { current sheets }\end{array}$ & $\begin{array}{l}\text { solar wind at } \\
\text { magnetosphere }^{c}\end{array}$ \\
\hline density $n, \mathrm{~cm}^{-3}$ & .5 & $7 \times 10^{7}$ & 10 \\
temperature $T, e V$ & .7 & $10^{3}$ & 10 \\
plasma parameter $\Lambda$ & $4 \times 10^{9}$ & $2 \times 10^{10}$ & $5 \times 10^{10}$ \\
ion thermal velocity $v_{t h, i}, \mathrm{~cm} / \mathrm{s}$ & $10^{6}$ & $3 \times 10^{7}$ & $5 \times 10^{6}$ \\
ion mean-free-path $\ell_{m f p, i}, \mathrm{~cm}$ & $6 \times 10^{11}$ & $10^{10}$ & $7 \times 10^{12}$ \\
magnetic diffusivity $\lambda, \mathrm{cm}^{2} / \mathrm{s}$ & $10^{7}$ & $8 \times 10^{2}$ & $6 \times 10^{5}$ \\
\hline magnetic field $B, G$ & $10^{-6}$ & 1 & $10^{-4}$ \\
plasma beta $\beta$ & 14 & 3 & 1 \\
Alfvén speed $v_{A}, \mathrm{~cm} / \mathrm{s}$ & $3 \times 10^{5}$ & $3 \times 10^{7}$ & $7 \times 10^{6}$ \\
ion gyroradius $\rho_{i}, \mathrm{~cm}$ & $10^{8}$ & $3 \times 10^{3}$ & $6 \times 10^{6}$ \\
\hline large-scale velocity $U, \mathrm{~cm} / \mathrm{s}$ & $10^{6}$ & $4 \times 10^{6}$ & $5 \times 10^{6}$ \\
large length scale $L, \mathrm{~cm}$ & $10^{20}$ & $5 \times 10^{10}$ & $10^{8}$ \\
Lundquist number $S_{L}=\frac{v_{A} L}{\lambda}$ & $3 \times 10^{18}$ & $2 \times 10^{15}$ & $10^{9}$ \\
resistive length $\ell_{\eta}^{\perp}, \mathrm{cm}$ & $5 \times 10^{5}$ & 1 & 20 \\
\hline
\end{tabular}

(Norman and Ferrara (1996); Ferrière (2001) $\quad$ Bemporad (2008) $\quad$ Zimbardo et al. (2010)

*This nominal resistive scale is calculated from $\ell_{\eta}^{\perp} \simeq L\left(v_{A} / U\right) S_{L}^{-3 / 4}$, assuming GS95 turbulence holds down to that scale, and should not be taken literally when $\ell_{\eta}^{\perp}<\rho_{i}$.

sinks of the energy are correctly identified, we can describe turbulence for arbitrary $R e$ and $R m$. The simplest description of the complex spatial variations of any physical variable, $X(\mathbf{r})$, is related to the amount of change of $X$ between points separated by a chosen displacement $\mathbf{l}$, averaged over the entire volume of interest. Usually the result is given in terms of the Fourier transform of this average, with the displacement $\mathbf{l}$ being replaced by the wavenumber $\mathbf{k}$ parallel to $\mathbf{l}$ and $|\mathbf{k}|=1 /|\mathbf{I}|$. For example, for isotropic turbulence the kinetic energy spectrum, $E(k) d k$, characterizes how much energy resides at the interval $k, k+d k$. At some large scale $L$ (i.e., small $k$ ), one expects to observe features reflecting energy injection. At small scales, energy dissipation should be seen. Between these two scales we expect to see a selfsimilar power-law scaling reflecting the process of non-linear energy transfer.

Thus, in spite of its complexity, the turbulent cascade is self-similar over its inertial range. The physical variables are proportional to simple powers of the eddy sizes over a large range of sizes, leading to scaling laws expressing the dependence of certain non-dimensional combinations of physical variables on the eddy size. Robust scaling relations can predict turbulent properties on the whole range of scales, including those that no large-scale numerical simulation can hope to resolve. These scaling relations are extremely important for obtaining an insight of the processes at the small scales.

The presence of a magnetic field makes MHD turbulence anisotropic (Montgomery and Turner 1981; Matthaeus et al. 1983; Shebalin et al. 1983; Higdon 1984; Goldreich and Sridhar 1995, see Oughton et al., 2003 for a review). The relative importance of hydrodynamic and magnetic forces changes with scale, so the anisotropy of MHD turbulence does too.
Many astrophysical results, e.g. the dynamics of dust, scattering and acceleration of energetic particles, thermal conduction, can be obtained if the turbulence spectrum and its anisotropy are known. As we discuss below, additional important insight can be obtained if we know turbulence intermittency.

Estimates of turbulence anisotropy obtained in relation to the observations of magnetic fluctuation of the outer heliosphere and solar wind (see Zank and Matthaeus 1992b, and references therein) provided, for an extended period of time, the only guidance for theoretical advances. This resulted in a picture of MHD turbulence consisting of 2D "reduced MHD" perturbations carrying approximately $80 \%$ of energy and "slab" Alfvénic waves carrying the remaining $20 \%$ of energy (see Matthaeus et al. 2002, and references therein). In other words, in the suggested picture the MHD turbulence was presented by two anisotropic components, one having wave vectors mostly perpendicular to magnetic field (the $2 \mathrm{D}$ one), the other having them mostly parallel to magnetic field (the slab one). This model became a default one for many calculations of the propagation of cosmic rays (see Bieber et al. 1988, 1994). On the contrary, guided mostly by compressible MHD numerical simulations, the interstellar community adopted a model of the MHD turbulence where the basic MHD modes, i.e. slow, fast and Alfvénic are well coupled together and efficiently dissipate energy in shocks (Stone et al. 1998; Mac Low 1999). Little cross-talk between the two communities did not stimulate the interdisciplinary debates on the nature of MHD turbulence, which was regretful, as the heliospheric community has the advantage of the in-situ spacecraft measurements.

In spite of the intrinsic limitations of the "brute force" approach, we feel that reliable results can be obtained nu- 
merically if the studies are focused on a particular property of turbulence in order to get a clear picture of the underlying physics occurring on small scales ("microphysics") that cannot be resolved in "global" interstellar simulations 4 .

For instance, numerical studies in Cho and Lazarian 2002, 2003) showed that the Alfvénic turbulence develops an independent cascade which is marginally affected by the fluid compressibility. This observation corresponds to theoretical expectations of the GS95 theory that we briefly describe below (see also Lithwick and Goldreich 2001). In this respect we note that the MHD approximation is widely used to describe the actual magnetized plasma turbulence over scales that are much larger than both the mean free path of the particles and their Larmor radius (see Kulsrud 1983, 2005, and references therein). More generally, the most important incompressible Alfvénic part of the plasma motions can described by MHD even below the mean free path but on the scales larger than the Larmor radius.

We claim that, while having a long history of ideas, the theory of MHD turbulence has become testable recently due to the advent of numerical simulations (see Biskamp 2003) which confirmed (see Cho and Lazarian 2005, and references therein) the prediction of magnetized Alfvénic eddies being elongated in the direction of magnetic field (see Shebalin et al. 1983; Higdon 1984) and provided results consistent with the quantitative relations for the degree of eddy elongation obtained in GS95. Indeed, GS95 made predictions regarding relative motions parallel and perpendicular to $\mathbf{B}$ for Alfvénic turbulence. The model did not predict the generation of any "slab" modes and, instead of pure 2D Alfvénic modes, predicted that most of the Alfvénic energy is concentrated in the modes with a so-called "critical balance" between the parallel and perpendicular motions. The latter can be understood within intuitive picture where eddies mixing magnetic field perpendicular to its loca 5 direction induce Alfvénic waves with the period equal to the period of the eddy rotation. This results in the scale-dependent anisotropy of velocity and magnetic perturbations, with the anisotropy being larger for smaller eddies.

The hydrodynamic counterpart of the MHD turbulence theory is the famous Kolmogorov (1941) theory of turbulence. In the latter theory energy is injected at large scales, creating large eddies which correspond to large Re numbers and therefore do not dissipate energy through viscos-

\footnotetext{
4 By contrast, numerical simulations that deal with many physical conditions simultaneously cannot distinguish between the effects of different processes. Moreover, they inevitably have a more restricted interval of scales on which energy is injected by numerics, initial conditions, or boundary conditions. Their results are, therefore, difficult to interpret in physical terms.

5 The notion of the direction being local is critical. Small eddies are affected by magnetic field in their vicinity, rather than a global field. No universal scalings are possible to obtain in the frame of the mean magnetic field.
}

ity 6 but transfer energy to smaller eddies. The process continues until the cascade reaches the scales which are small enough that the energy is dissipated during one turnover time of the eddies at the corresponding scales. In the absence of compressibility the hydrodynamic cascade of energy is $\sim v_{l}^{2} / \tau_{\text {casc }, l}=$ const, where $v_{l}$ is the velocity at the scale $l$ and the cascading time for the eddies of size $l$ is $\tau_{\text {cask }, l} \approx l / v_{l}$. From this the well known relation $v_{l} \sim l^{1 / 3}$ follows.

A frequent mental picture that astrophysicists have of the Alfvénic turbulence is based of Alfvén waves with wave vectors along the magnetic field. This is not true for the strong Alfvénic turbulence which, similar to its hydrodynamic counterpart, can be described in terms of eddies?. However, contrary to Kolmogorov turbulence, in the presence of dynamically important magnetic field eddies become anisotropic. At the same time, one can imagine eddies mixing magnetic field lines perpendicular to the direction of magnetic field.

The nature of Alfvénic cascade is expressed through the critical balance condition in GS95 model of strong turbulence, namely,

$l_{\|}^{-1} V_{A} \sim l_{\perp}^{-1} v_{l}$,

where $v_{l}$ is the eddy velocity, while the $l_{\|}$and $l_{\perp}$ are, respectively, eddy scales parallel and perpendicular to the local direction of magnetic field. The critical balance condition states that the parallel size of an eddy is determined by the distance Alfvénic perturbation can propagate during the eddy turnover. The notion of local is importan 8 , as no universal relations exist if eddies are treated with respect to the global mean magnetic field (LV99; Cho and Vishniac 2000; Maron and Goldreich 2001; Lithwick and Goldreich 2001; Cho and Lazarian 2002).

The critical balance is the feature of the strong turbulence, which is the case when the turbulent energy is injected at $V_{A}$. If the energy is injected at velocities lower than $V_{A}$ the cascade is weak with $l_{\perp}$ of the eddies increasing while $l_{\|}$staying the same ( $\mathrm{Ng}$ and Bhattacharjee 1996; Lazarian and Vishniac 1999; Galtier et al. 2002). In other words, as a result of the weak cascade the eddies get thinner, but

\footnotetext{
6 Reynolds number $\operatorname{Re} \equiv L_{f} V / v=\left(V / L_{f}\right) /\left(v / L_{f}^{2}\right)$ which is the ratio of an eddy turnover rate $\tau_{e d d y}^{-1}=V / L_{f}$ and the viscous dissipation rate $\tau_{d i s}^{-1}=\eta / L_{f}^{2}$. Therefore large $R e$ correspond to negligible viscous dissipation of large eddies over the cascading time $\tau_{\text {casc }}$ which is equal to $\tau_{e d d y}$ in Kolmogorov turbulence.

7 The description in terms of interacting wave packets or modes is also possible with the corresponding wave vectors tending to get more and more perpendicular to the magnetic field as the cascade develops.

8 To stress the difference between local and global systems here we do not use the language of $k$-vectors. Wave vectors parallel and perpendicular to magnetic fields can be used, if only the wave vectors are understood in terms of a wavelet transform defined with the local reference system rather than ordinary Fourier transform defined with the mean field system.
} 
Table 2 Regimes and ranges of MHD turbulence.

\begin{tabular}{lllll}
\hline \hline $\begin{array}{l}\text { Type } \\
\text { of MHD turbulence }\end{array}$ & $\begin{array}{l}\text { Injection } \\
\text { velocity }\end{array}$ & $\begin{array}{l}\text { Range } \\
\text { of scales }\end{array}$ & $\begin{array}{l}\text { Motion } \\
\text { type }\end{array}$ & $\begin{array}{l}\text { Ways } \\
\text { of study }\end{array}$ \\
\hline Weak & $V_{L}<V_{A}$ & {$\left[L, l_{\text {trans }}\right]$} & wave-like & analytical \\
\hline $\begin{array}{l}\text { Strong } \\
\text { subAlfvénic }\end{array}$ & $V_{L}<V_{A}$ & {$\left[l_{\text {trans }}, l_{\text {min }}\right]$} & eddy-like & numerical \\
\hline $\begin{array}{l}\text { Strong } \\
\text { superAlfvénic }\end{array}$ & $V_{L}>V_{A}$ & {$\left[l_{A}, l_{\text {min }}\right]$} & eddy-like & numerical \\
\hline
\end{tabular}

$L$ and $l_{\min }$ are injection and dissipation scales

$l_{\text {trans }}$ and $l_{A}$ are given by Eq. 5] and Eq. (6), respectively.

preserve the same length along the local magnetic field. This decreases $l_{\perp}$ and eventually makes Eq. (2) satisfied. If the injection velocity is $V_{L}$ and turbulent injection scale is $L$, the transition to the strong MHD turbulence happens at the scale $l\left(v_{l} / V_{A}\right)^{2}$ and the velocity at this scale is $V_{\text {strong }}=$ $V_{A}\left(v_{l} / V_{A}\right)^{2}$ (LV99; Lazarian 2006). Thus the weak turbulence has a limited, i.e. $\left[l, l\left(v_{l} / V_{A}\right)^{2}\right]$ inertial interval and get strong at smaller scales.

While GS95 assumed that the turbulent energy is injected at $V_{A}$ at the injection scale $l$, LV99 provided general relations for the turbulent scaling at small scales for the case that the injection velocity $V_{L}$ is less than or equal to $V_{A}$, which can be written in terms of $l_{\|}$and $l_{\perp}$ :

$l_{\|} \approx l\left(\frac{l_{\perp}}{l}\right)^{2 / 3}\left(\frac{V_{A}}{V_{L}}\right)^{4 / 3}$

$v_{l} \approx V_{L}\left(\frac{l}{L}\right)^{1 / 3}\left(\frac{V_{L}}{V_{A}}\right)^{1 / 3}$

It is important to stress that the scales $l_{\perp}$ and $l_{\|}$are measured with respect to the system of reference related to the direction of the local magnetic field "seen" by the eddy. This notion was not present in the original formulation of the GS95 theory and was added to it in LV99. The local system of reference was later used in numerical studies in Cho and Vishniac (2000), Maron and Goldreich (2001), and Cho et al. (2002) testing GS95 theory. In terms of mixing motions, it is rather obvious that the free Kolmogorov-type mixing is possible only with respect to the local magnetic field of the eddy rather than the mean magnetic field of the flow.

The original GS95 picture deals with transAlfvénic turbulence, i.e. with $V_{A} \sim V_{L}$. For low Alfvénic Mach numbers, i.e. for $V_{A} \gg V_{L}$ at large scales $\sim L$ the turbulence is weak (see Ng and Bhattacharjee 1997; Lazarian and Vishniac 1999; Galtier et al.2000) and magnetic fields are slightly perturbed by propagating Alfvén waves. The wave packets in weak turbulence evolve changing their perpendicular scale $l_{\perp}$, while their scale $l_{\|}$along the magnetic field does not change. As scaling of weak turbulence predicts $V_{l} \sim V_{L}\left(l_{\perp} / L\right)^{1 / 2}$ (LV99), at the scale

$l_{\text {trans }} \sim L\left(V_{L} / V_{A}\right)^{2} \equiv L M_{A}^{2}$ the critical balance condition $l_{\|} / V_{A} \approx l_{\perp} / V_{l}$ is getting satisfied making turbulence strong. It is easy to see that the velocity corresponding to $l_{\text {trans }}$ is $V_{\text {trans }} \sim V_{L}\left(V_{L} / V_{A}\right)$.

For superAlfvénic turbulence the situation is somewhat different. Magnetic field gets dynamically important as soon as its energy density exceeds the energy of eddies at the Ohmic dissipation scale, which translates into Alfvénic velocity getting larger than the velocity of eddies at the Ohmic dissipation scale or the ion Larmor radius, whichever is larger. As the velocity in Kolmogorov turbulence scale as $v_{l} \sim l^{1 / 3}$, it is clear that even weak magnetic field can make a significant impact on the dynamics of the smallest eddies. In view of that it is advantageous to introduce a scale at which the magnetic field gets dynamically important and the nature of the turbulence changes from hydrodynamic to MHD (see Lazarian 2006), namely,

$l_{A}=L\left(V_{A} / V_{L}\right)^{3}=L M_{A}^{-3}$

The relations predicted in GS95 were confirmed numerically for incompressible (Cho and Vishniac 2000; Maron and Goldreich 2001; Cho et al. 2002; Beresnyak and Lazarian 2009, 2010; Beresnyak 2011) and compressible MHD turbulence 9 (Cho and Lazarian 2002, 2003; Kowal and Lazarian 2010, see also Cho et al. 2003 , for a review). They are in good agreement with observed and inferred astrophysical spectra. A remarkable fact revealed in Cho et al. (2002) is that fluid motions perpendicular to $\mathbf{B}$ are identical to hydrodynamic motions. This provides an essential physical insight and explains why in some respects MHD turbulence and hydrodynamic turbulence are similar, while in other respects they are different.

GS95 provided theoretical arguments in favor of weak coupling between fast and Alfvén modes, and low impact of slow modes to Alfvén modes (see also Lithwick and Goldreich 2001). This challenged the paradigm accepted by the interstellar community. While the decomposition of MHD perturbations into fundamental MHD waves was widely used in

9 Some studies of MHD compressible turbulence, e.g. Vestuto et al. (2003) did not perform a decomposition of MHD perturbations into Alfvén, slow and fast modes as it is done in Cho and Lazarian (2002, 2003). They did not use local system of reference for which the GS95 scaling is formulated. Therefore a direct comparison of their results with the GS95 predictions is difficult. 
the literature (see Dobrowolny et al. 1980) it was usually assumed that the Alfvénic waves exist and interact with other waves for many periods (see a discussion in Zweibel 2003). This is not the case of the GS95 model of turbulence, where the Alfvén modes non-linearly decay within one wave period. This reduces the time of interaction and therefore the coupling. Interestingly enough, in GS95 model, the Alfvénic modes can affect slow modes, but the opposite is not true. These results were successfully tested in Cho and Lazarian (2002, 2003) and Kowal and Lazarian (2010).

Some of the relevant results are illustrated in Figure 2. Contrary to the common expectation, the modes exhibited nice scaling laws that allow further analytical and numerical applications. For instance, numerical studies in Cho and Lazarian (2002, 2003) revealed that the GS95 scalings are valid for the Alfvénic part of the turbulence cascade even in the highly compressible regime (see also Beresnyak and Lazarian 2006).

The statistical decomposition of MHD turbulence into Alfvén, slow and fast modes suggested in Cho and Lazarian (2002) was successfully tested for slow modes of magnetically dominated plasmas in Cho and Lazarian (2003). The decomposition of MHD turbulent motions into the discussed three cascades of fundamental modes have been confirmed by Kowal and Lazarian (2010), where in order to improve the decomposition, the wavelet transformation were used. The large scale components (large with respect to the size of the local wavelet), which determine the vector base of mode projection in Fourier space, are obtained by averaging over the size of the wavelet. Therefore, the wavelet transformations allow to approach closer to the decomposition in the local system of reference than the earlier statistical approach based on Fourier analysis could do. In Figure 3 we show that the new wavelet decomposition results (upper row) are very similar to the ones obtained with the Fourier method (lower row). At the same time, the study of spectra and anisotropy of velocity, magnetic field reveals the advantages of the wavelets in comparison with the Fourier technique for studying turbulence with weak mean field. For turbulence with $B_{\text {mean }} \sim \delta B$ the obtained results are consistent with the Cho and Lazarian 2002, 2003) studies.

Nevertheless, it would be wrong to say that we have a complete understanding of the scaling of MHD modes and their interactions. First of all, one should distinguish weak and strong Alfvénic turbulence. The weak turbulence is essentially $2 \mathrm{D} 10$, with the turbulent cascade creating more structure perpendicular to magnetic field as the turbulence cascades (see Galtier et al. 2000). Such a cascade emerges when the driving of turbulence at the outer scale is weak, i.e. the injection velocity is much less than the Alfvén velocity. Although the weak turbulence picture corresponds to the early representation of MHD turbulence (see discussion in

\footnotetext{
10 Practical studies of non-linearity of turbulence in Solar wind are discussed in Sahraoui and Goldstein (2010); Ghosh et al. (2011).
}

$\S 2.1$, one should keep in mind that the strength of Alfvénic interactions increases with the decrease of the scale along the cascade. Therefore the Alfvénic turbulence gets eventually strong, while both the inertial range and the astrophysical utility of the weak Alfvénic cascade are limited. The interaction of weak Alfvénic turbulence with fast modes has dependences on the angle between $\mathbf{B}$ and the wave vector (Chandran 2005).

As the Mach number of turbulence increases, shocks should play more important role in the dynamics of compressible motions. The transition from the regime when compressible motions can be well described by the fast and slow mode cascades versus the situation when shocks carry an appreciable part of the energy deserves more studies. A study in Beresnyak et al. (2005, henceforth BLC05) shows that in the space between shocks the description of the perturbations with slow modes is valid for all the Mach numbers studied (up to 10).

The total velocity field contains two components: solenoidal, which is equivalent to the incompressible part, and potential, which contains the compressible part of the field and the remaining part which is curl and divergence free. In Table 3 , we show the percentage contribution of each component to the total velocity field. We see that the compressible part constitutes only a fraction of the total field. However, the magnitude of this fraction is different for sub and supersonic models. In the case of sub-Alfvénic turbulence, it is about $3 \%$ in subsonic models and about $7 \%$ in supersonic models, which confirms a higher efficiency of the compression in the presence of supersonic flows. Furthermore, the fraction also changes when we compare models with strong and weak magnetic fields. The velocity field, in the presence of a weak magnetic field, contains about $5 \%$ of the compressible part in the model with $\mathscr{M}_{s} \sim 0.7$ and even up to $16 \%$ in models with $\mathscr{M}_{s}>1$. The consequence of the presence of a strong magnetic field results in a reduction of the compressible part of the velocity field by a factor of 2 . This indicates a substantial role of the magnetic field in the damping of the generation of the compressible flows.

The wavelet decomposition, important for MHD turbulence, separates the velocity field into three different MHD waves: an incompressible Alfvén wave and slow and fast magneto acoustic waves, of both which are compressible. In Table 3, we included the percentage amount of these components in the total velocity field. As we see, most of the energy is contained in the Alfvén wave. It is almost $60 \%$ in the case of sub-Alfvén turbulence, and about $50 \%$ for superAlfvénic turbulence. The slow wave contains approximately $1 / 3$ of the total energy. However, for the super-Alfvénic case, this amount is slightly higher. Table 3 suggests that the slow wave is weaker when the turbulence becomes supersonic. We do not see a similar behavior for the Alfvén wave in the case of models with a strong magnetic field. This effect 


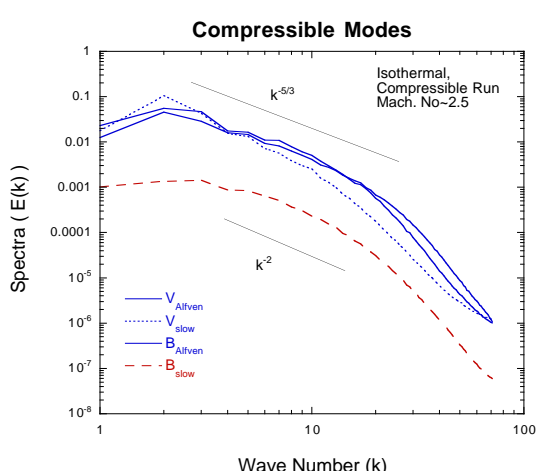

(a)

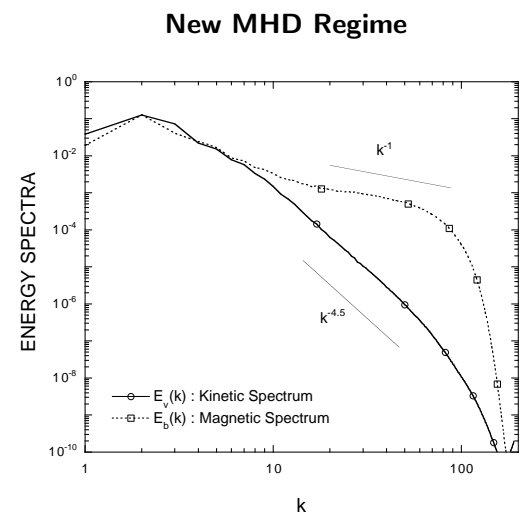

(d)

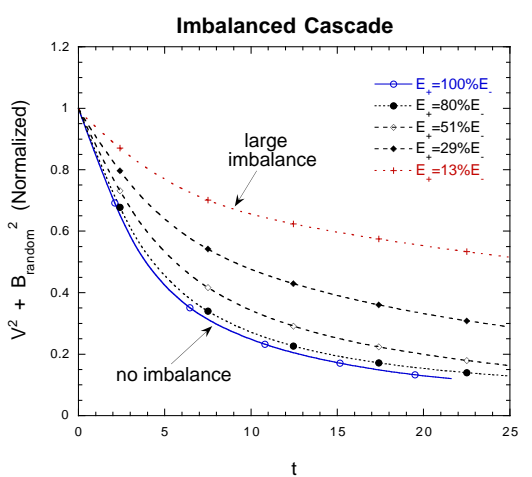

(b)

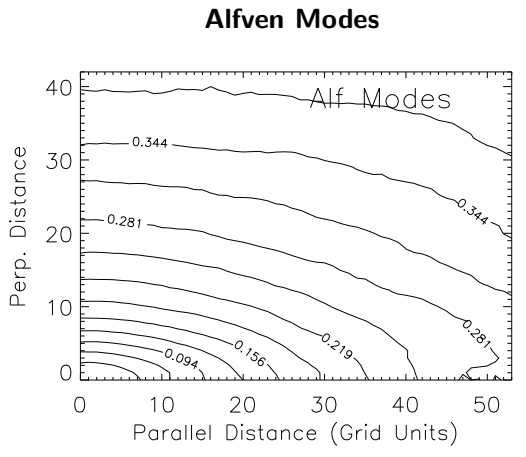

(e)

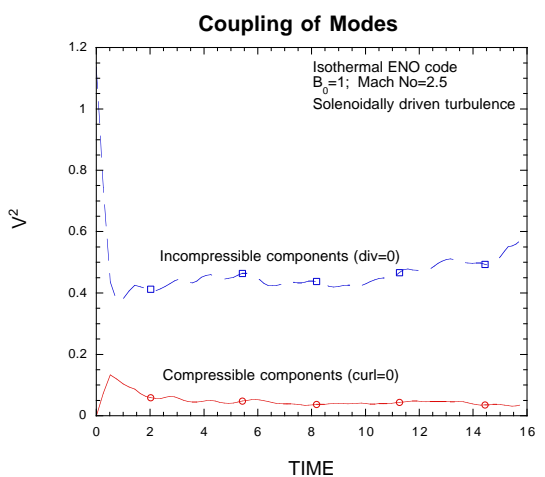

(c)

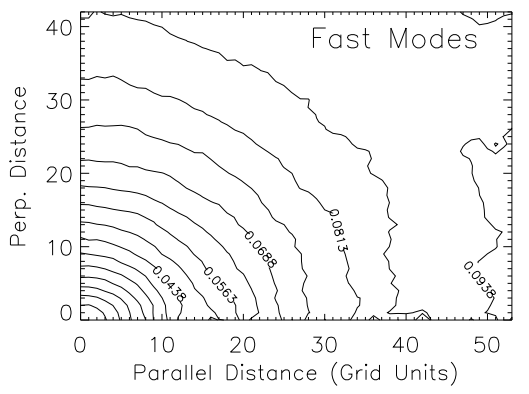

(f)

Fig. 2 Statistics of MHD Turbulence: (a). Scaling of compressible motions for plasma with magnetic pressure ten times the gas pressure. This regime is important for molecular clouds (from CL02). The velocity (solid line) and B (dashed line) spectra are plotted against $k$ (三1/(eddy size)). They show well defined statistical properties that allow further fruitful applications. (b). The simulations of decaying turbulence show that the rate of the decay of the total energy is a strong function of the imbalance of the energy contained in waves initially moving in opposite directions. In the lowest curve, the waves have the same amplitude. The energy decays more slowly with imbalance (Cho et al. 2002). (c). The evolution of kinetic energy in 3-D compressible turbulence, initially started with $\nabla \cdot v=0$ as if the gas were incompressible. The dashed curve shows the evolution of the $\nabla \cdot v=0$ motions. We see that Alfvénic turbulence creates only a marginal amount of compressible motions, suggesting that Alfvénic modes should evolve independently of the compressible cascade (CLO2). (d). Magnetic fluctuations persist beyond the turbulent damping scale at large $k$, while hydrodynamic fluctuations damp out in partially ionized gas (Lazarian et al. 2004). This viscosity-dominated regime of turbulence may dominate small scale structure of partially ionized gas. (e-f). Isocontours of equal correlation for Alfvén and fast modes (CL02). (e) The Alfvénic motions are much more correlated along $\mathbf{B}$ than perpendicular to it. (f) In contrast, fast magnetosonic fluctuations show essentially circular (isotropic) isocontours of correlation.

could also take place in the super-Alfvénic models, but it is weakened by relatively large errors. An interesting dependence is observed in the case of the fast wave. Although the fast wave is the weakest among all MHD waves, it strongly depends on the regime of turbulence. Similarly to the compressible part of the velocity field, it is stronger for models with a weak magnetic field. In addition, it is much stronger when turbulence is supersonic, but this strength seems to be weakly dependent on the sonic Mach number.

\subsection{Density structure of MHD turbulent flows}

Density structure of turbulence is usually discussed in relation to star formation studies. However, we shall show in 86 that fluctuations of density play important role for energetic particle acceleration. Thus it is important to survey the basic properties of the turbulent density field.

The power spectrum of density fluctuations is an important property of a compressible flow. In some cases, the spectrum of density can be derived analytically. For nearly incompressible turbulent motions in the presence of a strong magnetic field, the spectrum of density scales similarly to the pressure, i.e. $E_{\rho}(k) \sim k^{-7 / 3}$ if we consider the polytropic equation of state $p=a \rho^{\gamma}$ (Biskamp 2003). In weakly magnetized nearly incompressible MHD turbulence, however, velocities convect density fluctuations passively inducing the spectrum $E_{\rho}(k) \sim k^{-5 / 3}$ (Montgomery et al. 1987). In supersonic flows, these relations are not valid anymore because of shocks accumulating matter into the local and highly dense structures. Due to the high contrast of density, 

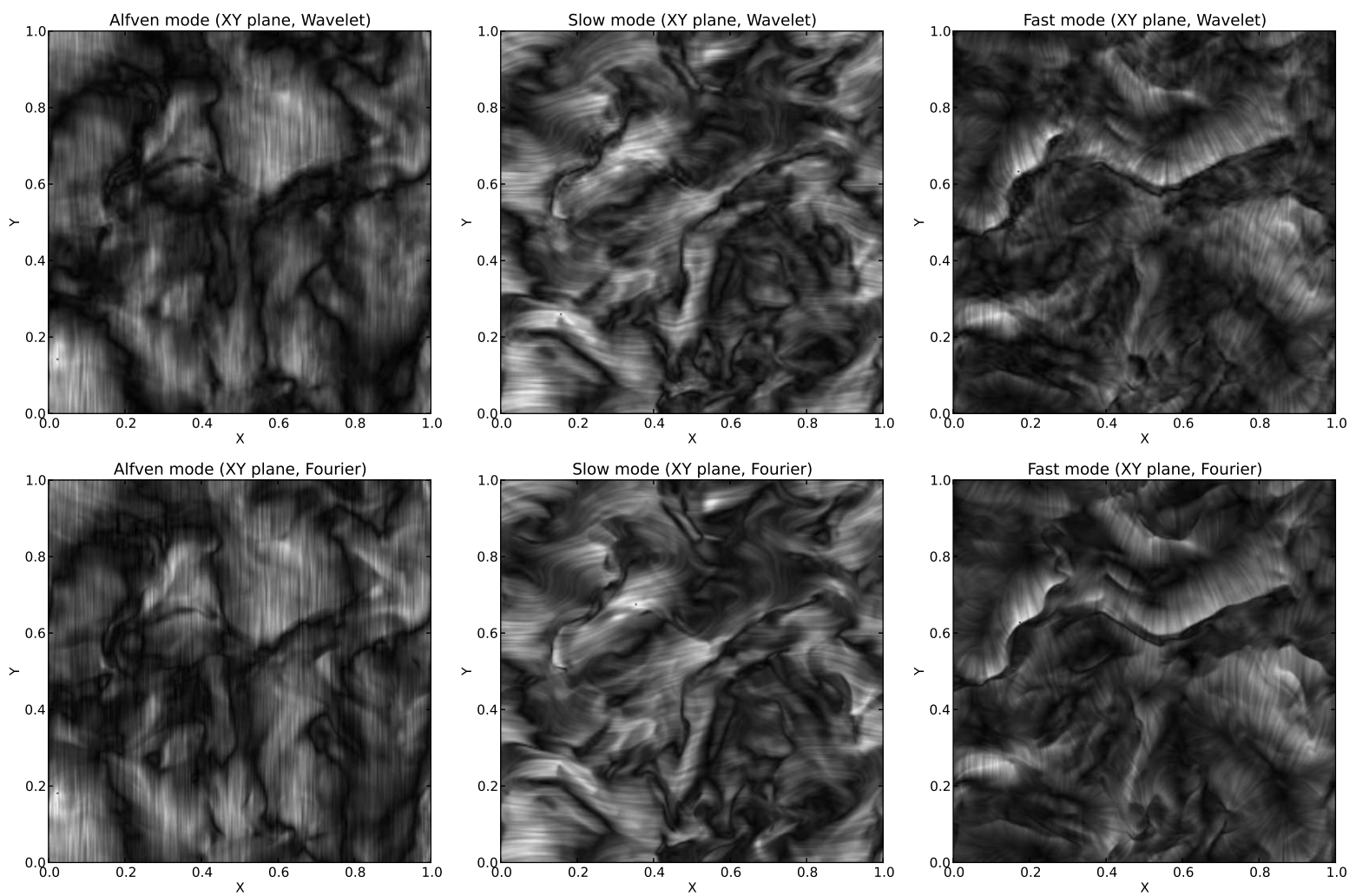

Fig. 3 The topology of the Alfvén, slow and fast mode (left, middle, and right column, respectively) in the plane parallel to the mean field. The upper and lower rows show the MHD modes separated using the wavelet (Kowal and Lazarian 2010) and Fourier (Cho and Lazarian 2003) methods, respectively. Texture shows the direction of velocity field multiplied by the local amplitude.

Table 3 Percentage Amount of the Kinetic Energy Contained Within Each Velocity Component in Kowal and Lazarian (2010). Errors correspond to a measure of the time variation.

\begin{tabular}{cc|cc|ccc}
$\mathscr{M}_{s}$ & $\mathscr{M}_{A}$ & $V_{\text {incomp. }}$ & $V_{\text {comp. }}$ & $V_{A}$ & $V_{s}$ & $V_{f}$ \\
\hline$\sim 0.8$ & $\sim 0.7$ & $96.5^{ \pm 0.8}$ & $3.3^{ \pm 0.8}$ & $58^{ \pm 4}$ & $37^{ \pm 3}$ & $4.8^{ \pm 0.7}$ \\
$\sim 2.5$ & $\sim 0.6$ & $93^{ \pm 2}$ & $7^{ \pm 2}$ & $58^{ \pm 5}$ & $33^{ \pm 4}$ & $9^{ \pm 2}$ \\
$\sim 7.5$ & $\sim 0.5$ & $92^{ \pm 2}$ & $7^{ \pm 2}$ & $56^{ \pm 4}$ & $36^{ \pm 4}$ & $8.0^{ \pm 0.7}$ \\
$\sim 0.7$ & $\sim 2.1$ & $95^{ \pm 2}$ & $5^{ \pm 2}$ & $52^{ \pm 4}$ & $42^{ \pm 4}$ & $6.2^{ \pm 0.8}$ \\
$\sim 2.5$ & $\sim 2.1$ & $86^{ \pm 1}$ & $14^{ \pm 2}$ & $47^{ \pm 3}$ & $37^{ \pm 4}$ & $16^{ \pm 2}$ \\
$\sim 7.5$ & $\sim 1.9$ & $84^{ \pm 2}$ & $16^{ \pm 2}$ & $47^{ \pm 4}$ & $33^{ \pm 4}$ & $20^{ \pm 2}$
\end{tabular}

the linear relation $\delta p=c_{s}^{2} \delta \rho$ is no longer valid, and the spectrum of density cannot be related to pressure straightforwardly.

In Figure 4 we present the power spectra of fluctuations of density for models with different $\mathscr{M}_{s}$. As expected, we note a strong growth of the amplitude of density fluctuations with the sonic Mach number at all scales. This behavior is observed both in sub-Alfvénic as well as in super-Alfvénic turbulence (see Fig. 4). In Table 4 the spectral index of density $\alpha_{\rho}$ and the $\log$ arithm of density $\alpha_{\log \rho}$ is presented. The width of the inertial range is shown by the range of solid lines with slopes $-5 / 3$ and -2 in all spectra plots. Table 4 also shows the errors of estimation which combine the error of the fitting of the spectral index at each time snapshot and the standard deviation of variance of $\alpha_{\rho, \log \rho}$ in time. The slopes of the density spectra do not change significantly with $\mathscr{M}_{s}$ for subsonic experiments and correspond to analytical estimations (about -2.2 , which is slightly less than $-7 / 3$, for turbulence with $\mathscr{M}_{A} \sim 0.7$ and about -1.7 , which is slightly more than $-5 / 3$, for weakly magnetized turbulence with $\mathscr{M}_{A} \sim 7.3$ ). Such an agreement confirms the validity of the theoretical approximations. Those, nevertheless, do not cover the entire parameter space. While the fluid motions become supersonic, they strongly influence the density structure, making the small-scale structures more pronounced, which implies flattening of the spectra of density fluctuations (see values for $\mathscr{M}_{s}>1.0$ in Table 4 , see also Beresnyak et al. 2005). 

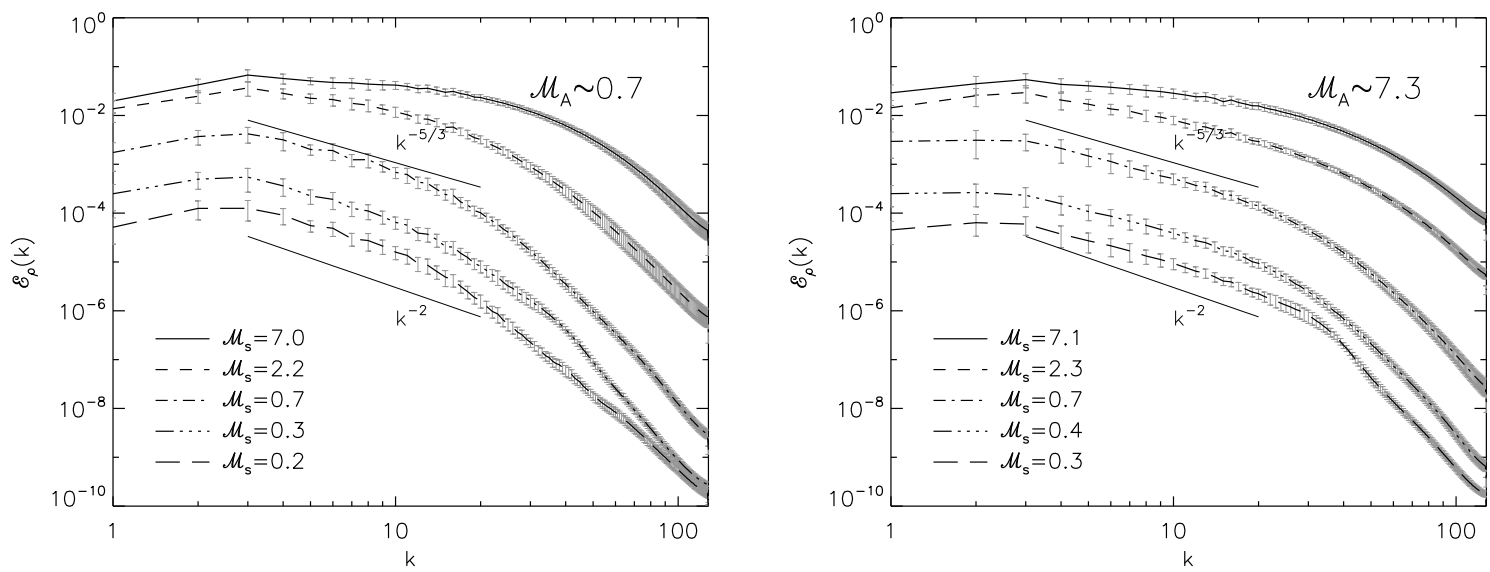

Fig. 4 Spectra of density for experiments with different values of $\mathscr{M}_{s}$ and with $\mathscr{M}_{A} \sim 0.7$ (left panel) and $\mathscr{M}_{A} \sim 7.3$ (right panel) for models with medium resolution $\left(256^{3}\right)$. Grey error bars signify the variance of spectra with time. The solid lines with slopes $-5 / 3$ and -2 cover the inertial range used to estimate the spectral indices of $\rho$ spectra. From Kowal et al. (2007).

Table 4 Slopes of the Power Spectrum of Density and the Logarithm of Density Fluctuations in Kowal et al. (2007).

\begin{tabular}{ccc|ccc}
\multicolumn{3}{c|}{$\mathscr{M}_{A} \sim 0.7$} & \multicolumn{3}{c}{$\mathscr{M}_{A} \sim 7$} \\
$\mathscr{M}_{s}$ & $\alpha_{\rho}$ & $\alpha_{\log \rho}$ & $\mathscr{M}_{s}$ & $\alpha_{\rho}$ & $\alpha_{\log \rho}$ \\
\hline $0.23^{ \pm 0.01}$ & $-2.3^{ \pm 0.3}$ & $-2.3^{ \pm 0.3}$ & $0.26^{ \pm 0.03}$ & $-1.7^{ \pm 0.3}$ & $-1.7^{ \pm 0.3}$ \\
$0.33^{ \pm 0.01}$ & $-2.2^{ \pm 0.3}$ & $-2.2^{ \pm 0.3}$ & $0.36^{ \pm 0.04}$ & $-1.7^{ \pm 0.3}$ & $-1.7^{ \pm 0.3}$ \\
$0.68^{ \pm 0.03}$ & $-2.0^{ \pm 0.3}$ & $-2.1^{ \pm 0.3}$ & $0.74^{ \pm 0.06}$ & $-1.6^{ \pm 0.2}$ & $-1.6^{ \pm 0.3}$ \\
$2.20^{ \pm 0.03}$ & $-1.3^{ \pm 0.2}$ & $-2.0^{ \pm 0.2}$ & $2.34^{ \pm 0.08}$ & $-1.2^{ \pm 0.2}$ & $-1.6^{ \pm 0.2}$ \\
$7.0^{ \pm 0.3}$ & $-0.5^{ \pm 0.1}$ & $-1.7^{ \pm 0.2}$ & $7.1^{ \pm 0.3}$ & $-0.6^{ \pm 0.2}$ & $-1.5^{ \pm 0.2}$
\end{tabular}

The values have been estimated within the inertial range for models with $\mathscr{M}_{A} \sim 0.7$ (left) and $\mathscr{M}_{A} \sim 7$ (right). Errors of spectral indices combine the errors of estimation at each time snapshot and the standard deviation of variance in time. Errors for sonic Mach numbers are the standard deviation of their variance in time calculated over the period starting from $t \geq 5$ to the last available snapshot.

Another question is the anisotropy of density and the logarithm of density structures. For subsonic turbulence it is natural to assume that the density anisotropies will mimic velocity anisotropies in the GS95 picture. This was confirmed in Cho et al. (2003), who observed that for supersonic turbulence the contours of density isocorrelation becomes round, corresponding to isotropy. Beresnyak et al. (2005 however, showed that anisotropies restore the GS95 form if instead of density one studies the logarithm of density. This is due to the suppression of the influence of the high density peaks, which arise from shocks. It is these peaks that mask the anisotropy of weaker, but more widely spread density fluctuations.

In Figure 5 we show lines that mark the corresponding separation lengths for the second-order structure functions parallel and perpendicular to the local mean magnetic field 11 In the case of subAlfvénic turbulence, the degree of

\footnotetext{
11 The local mean magnetic field was computed using the procedure of smoothing by a 3D Gaussian profile with the width equal to the separation length. Because the volume of smoothing grows with the separation length $l$, the direction of the local mean magnetic field might change with $l$ at an arbitrary point. This is an extension of the procedures employed in Cho et al. (2002).
}

anisotropy for density is very difficult to estimate due to the high dispersion of points. However, rough estimates suggest more isotropic density structures, because the points extend along the line $l_{\|} \sim l_{\perp}^{1}$. For models with $\mathscr{M}_{A} \sim 7.3$ the points in Figure 5 have lower dispersion, and the anisotropy is more like the type from GS95, i.e. $l_{\|} \sim l_{\perp}^{2 / 3}$. In both Alfvénic regimes, the anisotropy of density does not change significantly with $\mathscr{M}_{s}$. Plots for the logarithm of density show more smooth relations between parallel and perpendicular structure functions. The dispersion of points is very small. Moreover, we note the change of anisotropy with the scale. Lower values of structure functions correspond to lower values of the separation length (small-scale structures), so we might note that the logarithm of density structures are more isotropic than the GS95 model at small scales, but the anisotropy grows a bit larger than the GS95 prediction at larger scales. This difference is somewhat larger in the case of models with stronger external magnetic field (compare plots in the left and right columns of Figure 5, which may signify their dependence on the strength of $B_{\text {ext }}$. The anisotropy of $\log \rho$ structures is marginally dependent on the sonic Mach number, similar to the density structures. All these observations 

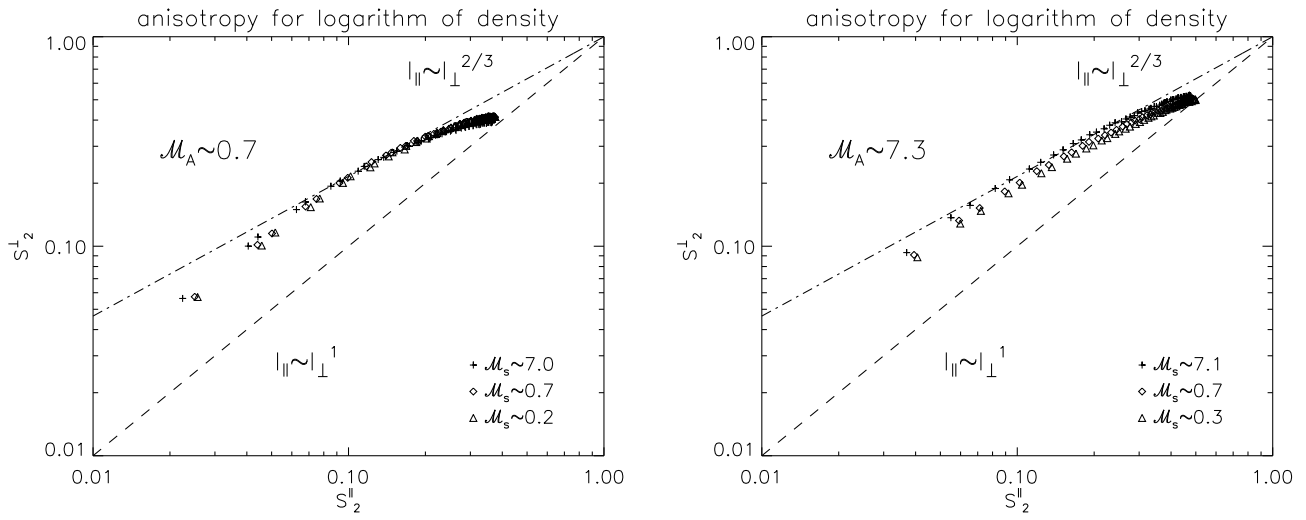

Fig. 5 Left: Anisotropy for the $2^{\text {nd }}$-order SF for the logarithm of density for $\mathscr{M}_{A} \sim 7.3$; Right: the same is for $\mathscr{M}_{A} \sim 7.3$. The logarithm of density traces the anisotropies of turbulent magnetic field. From Kowal et al. (2007).

allow us to confirm the previous studies (see Beresnyak et al. 2005) that suggest that the anisotropy depends not only on the scale but on $\mathscr{M}_{A}$.

Probability distribution functions (PDFs) of density and column density give us information about the fraction of the total volume occupied by a given value of a measured quantity. For the case of compressible turbulence in which evolution is described by the Navier-Stokes equation and the isothermal equation of state, the PDF of density obeys a lognormal form (Passot and Vázquez-Semadeni 1998). If we select a point in space and assume that the density at this point results from subsequent events perturbing the previous density, then the final density is a product $\rho_{0} \Pi_{i}\left(1+\delta_{i}\right)$, where $\rho_{0}$ is the initial density at the selected point and $\delta_{i}$ is a small compression/rarefaction factor. By the power of the Central Limit Theorem, the logarithm of the resulting density, $\log \left(\rho / \rho_{0}\right)=\sum_{i} \log \left(1+\delta_{i}\right)$, should obey a Gaussian distribution.

It was discussed in Vázquez-Semadeni and García (2001) that a parameter, which we for our convenience denote $\boldsymbol{\aleph}$, can determine the form of the column density PDF in molecular clouds. This parameter is the ratio of the cloud size to the decorrelation length of the density field. They defined the decorrelation length as the lag at which the density autocorrelation function (ACF) has decayed to its $10 \%$ level. If the density perturbation events are uncorrelated for $\boldsymbol{\aleph}>1$, large values of the ratio $\boldsymbol{\aleph}$ imply that the Central Limit Theorem can be applied to those events.

The decorrelation length estimated from the ACF of the density for our models ranges from about 20 cell sizes of the computational mesh for supersonic models to about 50 cell sizes for subsonic models, which corresponds to $5<\boldsymbol{\aleph}<13$ if we take the size of the computational box as the column length (in the case of medium resolution, it is equal to 256 cell sizes). Found values of $\boldsymbol{N}$ signify that in our models at least partial convergence to a Gaussian PDF should occur.
In the top row of Figure 6 we show PDFs of density normalized by its mean value for all models with medium resolution $\left(256^{3}\right)$. The plot on the left shows results obtained from sub-Alfvénic experiments $\left(\mathscr{M}_{A} \sim 0.7\right)$, and the plot on the right results from super-Alfvénic models $\left(\mathscr{M}_{A} \sim 7.3\right)$.

Our plots confirm the strong dependence of PDFs of density on the sonic Mach number, an already known and well-studied property of density fluctuations in compressible turbulence (see Vázquez-Semadeni and García 2001; Ostriker 2003, and references therein). For most of the models PDFs are lognormal functions. For super-Alfvénic turbulence PDFs are very symmetric about a vertical line crossing their maxima. However, for models with a strong external magnetic field (sub-Alfvénic turbulence, $\mathscr{M}_{A} \sim 0.7$ ) and very low pressure (supersonic turbulence, $\mathscr{M}_{s}>1.0$ ), the shape of the density PDFs is significantly deformed, and its lower value arm ends in higher densities than in the case with a weak magnetic field (compare models for $\mathscr{M}_{s} \sim 7$ drawn with solid lines in the left and right plots of Figure 6). This supports a hypothesis that the gauge symmetry for $\log \rho$ that exists in Navier-Stokes equations is broken in MHD equations because of the magnetic tension term (Beresnyak et al. 2005), which physically manifests itself by preventing the formation of highly underdense regions. In the higher density part of the distribution we do not see a similar effect, because the highly dense structures are created mainly due to shocks, and in this case, the magnetic tension is too weak to prevent the condensation.

In Figure 6 we included the degree of variation of PDFs in time as grey error bars. We note that the departure of PDFs from their mean profiles is very small in the middle part around the mean value. The strongest time changes are observed in the low- and high-density tails, but the PDFs for different models are still separable.

According to Cho and Lazarian (2003), the relations between the variance of density fluctuations and the sonic Mach number are $\delta \rho / \rho_{0} \sim \mathscr{M}_{s}$ when the magnetic pressure dom- 

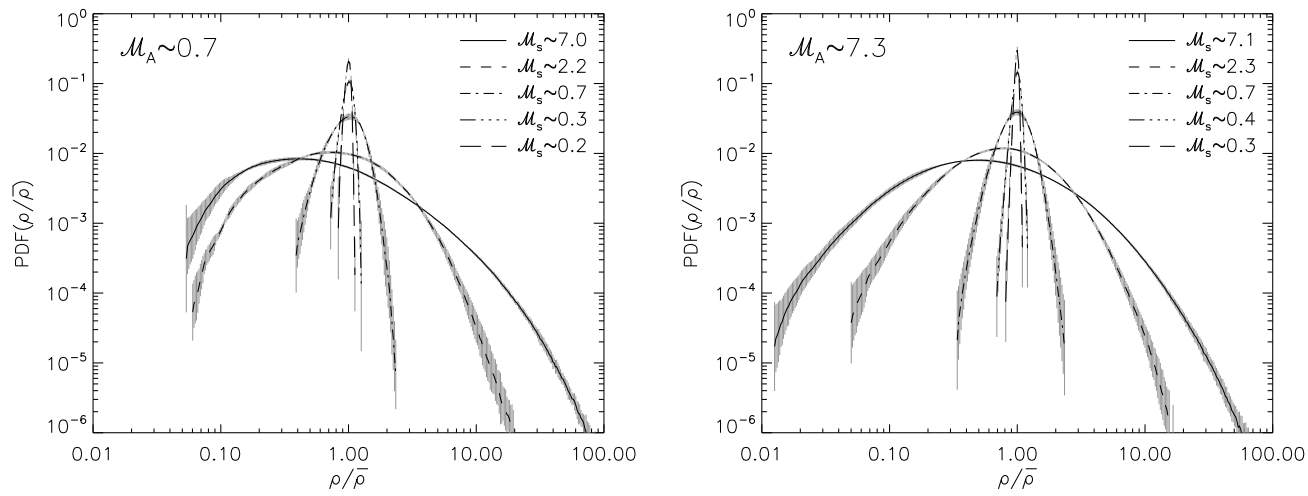

Fig. 6 Normalized probability distribution functions (PDFs) of density $\rho / \bar{\rho}$ (top row) for models with different values of $\mathscr{M}_{s}$ and for $\mathscr{M}_{A} \sim 0.7$ (left column) and $\mathscr{M}_{A} \sim 7.3$ (right column). Grey error bars signify the standard deviation of the PDFs, showing the strength of the departure at a single time snapshot from its mean profile averaged over time from $t \geq 5$ to the last available snapshot. From Kowal et al. (2007).

inates and $\delta \rho / \rho_{0} \sim \mathscr{M}_{s}^{2}$ when the gas pressure dominates. The former case is observed when $\mathscr{M}_{s} \gg \mathscr{M}_{A}$. Indeed, in Figure 7 we see that the mean standard deviation of the density fluctuations $\left\langle\delta \rho / \rho_{0}\right\rangle$ scales with $\mathscr{M}_{s}$ almost linearly when $\mathscr{M}_{s}>\mathscr{M}_{A}$. When $\mathscr{M}_{s}<\mathscr{M}_{A}$, the relation is much steeper. A similar behavior is observed in the case of superAlfvénic turbulence (Fig. 7 , right) although the gas pressure dominates in all models. For models with a very small value of the sonic Mach number, the relation is different. The value of $\left\langle\delta \rho / \rho_{0}\right\rangle$ depends much less on $\mathscr{M}_{s}$.

\section{Outstanding issues of MHD turbulence theory}

\subsection{The actual spectral slope of MHD turbulence}

Recently there has been a debate concerning an asymptotic scaling of MHD turbulence. While the GS95 theory predicts Kolmogorov's $-5 / 3$ scaling, the modification of theory in Boldvrev (2005, 2006) claim 12 , the power law index of $-3 / 2$. Beresnyak and Lazarian (2009, 2010) showed that MHD turbulence is less local then its hydrodynamic counterpart and therefore the shallow spectrum in simulations is expected as a result of an extended bottleneck intrinsic to MHD turbulence. Below we discuss more recent numerical tests related to this controversy.

One of the way to discriminate different models is by means of direct numerical simulations. A quantitative method called a resolution study is a traditional way to establish a correspondance between numerics and theory. This method has been used in the studies of hydrodynamic turbulence,

\footnotetext{
12 The model adds to GS95 the effect of the alignment of velocity and magnetic field, e.g. Ting et al. (1986) and which has been first numerically quantified in 3D numerical studies Beresnyak and Lazarian (2006). It requires that the dynamical alignment should increase with the decrease of the scale, which, however, contradicts to numerical calculations in Beresnyak (2012).
}

e.g. Yeung and Zhou (1997); Gotoh et al. (2002), etc. We perform several simulations with different Reynolds numbers. If we believe that turbulence is universal and the scale separation between forcing scale and dissipation scale is large enough, the properties of small scales should not depend on how turbulence was driven. This is because MHD equations and their numerical approximations contain no scale explicitly, save for the grid scale, so simulation with a smaller dissipation scale could be considered, as a simulation with the same dissipation scale, which is a multiple of grid scale, but larger driving scale. E.g., the small scale statistics in a $1024^{3}$ simulation should look the same as small-scale statistics in $512^{3}$, if the physical size of the elementary cell is the same and the dissipation scale is the same.

The above scaling argument requires that the geometry of the elementary cells is the same and the actual numerical scheme used to solve the equations is the same. The scaling argument does not require high precision on the dissipation scale or a particular form of dissipation, either explicit or numerical. This is because we only need the small-scale statistics to be similar in the particular series of simulations. This is achieved by applying the same numerical procedure. On the other hand, if turbulence is local, this ensures that small scales are only marginally affected by large scales. In practice, the scaling argument, which is also known as a resolution study, is done in the following way: the averaged spectra in two simulations are expressed in dimensionless units corresponding to the expected scaling, for example a $E(k) k^{5 / 3} \varepsilon^{-2 / 3}$ is used for hydrodynamics, and plotted versus dimensionless wavenumber $k \eta$, where dissipation scale $\eta$ correspond to the same model, e.g. $\eta=\left(v^{3} / \varepsilon\right)^{1 / 4}$ is used for scalar second order viscosity $v$ and Kolmogorov phenomenology. As long as the spectra are plotted this way and the scaling is correct, the curves obtained in simulations with different resolutions have to collapse into a single plot on small scales. Not only the spectrum, but any other statisti- 

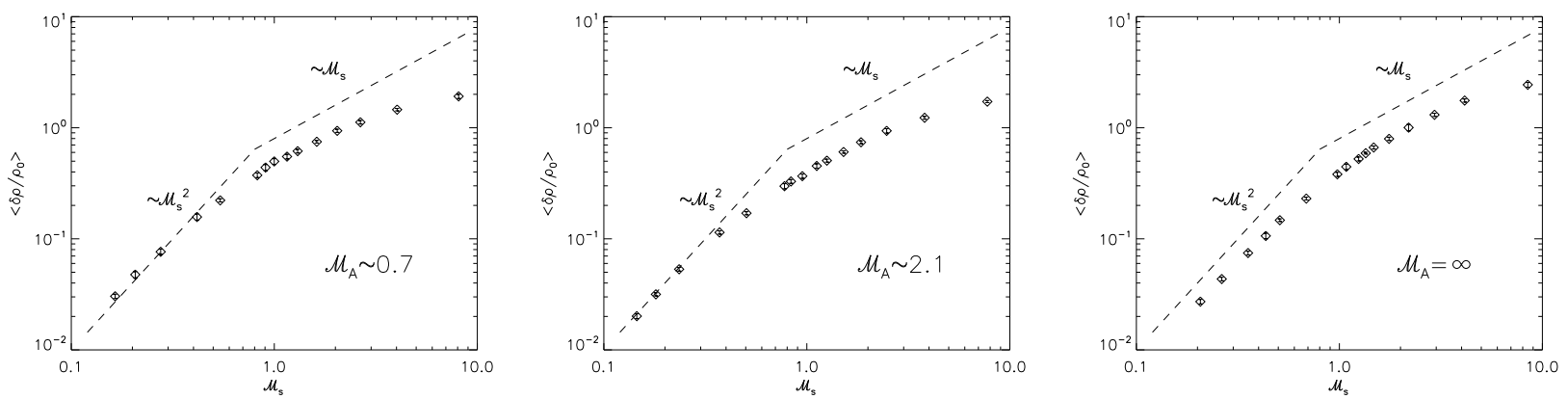

Fig. 7 Relation between the mean value of the standard deviation of density fluctuation $\left\langle\delta \rho / \rho_{0}\right\rangle$ and the sonic Mach number $\mathscr{M}_{s}$ for models with low resolution. Two lines show the analytical relations derived for magnetic-pressure-dominated turbulence $\left(\sim \mathscr{M}_{s}\right)$ and the gas-pressuredominated turbulence $\left(\sim \mathscr{M}_{s}^{2}\right)$.

cal property of turbulence can be treated this way. When the resolution is increasing, the convergence is supposed to become better. This is because of the increased separation of scale between driving and dissipation. The optimum strategy for our MHD study is to perform the largest resolution simulations possible and do the scaling study.

Figure 8 shows that the convergence study is suggestive of $-5 / 3$ spectral slope. The claims related to a more shallow slope are likely to be related to the lesser degree of locality of MHD turbulence, which results in the extended bottleneck region (Beresnyak and Lazarian 2010). The question definitely requires further studies, but we believe that at the moment there is not enough evidence to claim that GS95 model contradicts to simulations.

\subsection{Extended current sheets in viscosity dominated} turbulence

For high $P r_{m}$ fluid Cho et al. (2002) reported a new regime of MHD turbulence. Lazarian et al. (2004) showed that while the spectrum of volume-averaged magnetic fluctuations scales as $E_{b}(k) \sim k^{-1}$ (see Figure 9), the pressure within intermittent magnetic structures increases with the decrease of the scale $\propto k$ and the filling factor decreases $\propto k^{-1}$. The magnetic pressure compresses the gas as demonstrated in Figure 9 More importantly, extended current sheets that naturally emerge as magnetic field fluctuates in the plane perpendicular to the mean magnetic field (see Figure 9). It was speculated in Lazarian (2007) that these current sheets can account for the origin of the small ionized and neutral structures (SINS) on AU spatial scales Dieter et al. (1976); Heiles (1997); Stanimirović et al. (2004). These current sheets may present sites of cosmic ray acceleration.

Goldreich and Sridhar (2006) appealed not to high $\mathrm{Pr}_{m}$ MHD turbulence per se, but to the generation of the magnetic field in the turbulent plasma (see Schekochihin et al. 2004) to account for the high amplitude, but small scale fluctuations of plasma density observed in the direction of the Galactic center. We believe that the regime of dynamo in Schekochihin et al. (2004) and the turbulence in Lazarian et al. (2004) have similarities in terms of the density enhancement that are created. Although in the case of magnetic turbulence with sufficiently strong mean magnetic field, global reversals, that Goldreich and Sridhar (2006) appeal to in compressing plasma, do not happen, the reversals of the magnetic field direction occur in the direction perpendicular to the mean magnetic field. As the mean magnetic field goes to zero, the two regimes get indistinguishable. The regime of viscosity damped turbulence requires further systematic studies.

\subsection{Imbalanced MHD turbulence}

MHD turbulence in the presence of sources and sinks gets imbalanced, in the sense that the flow of energy in one direction is larger than the flow of energy in the opposite direction. Solar wind presents a vivid example of imbalanced turbulence with most waves near the Sun moving in the direction away from the Sun. While theories of balanced MHD turbulence enjoyed much attention, the theory of imbalanced turbulence 13 attracted less work, unfortunately. Important papers on imbalanced turbulence include (see Ting et al. 1986; Matthaeus et al. 1983; Biskamp 2003, and references therein). In terms of Solar wind observations one may mentioned studies by Roberts et al. (1987b 1987a), which showed that the imbalance of turbulence is not increasing contrary to the idealized theoretical expectations. The analytical results were obtained for weak imbalanced turbulence (Galtier et al. 2002; Lithwick and Goldreich 2003) and they are applicable in a rather narrow range of imbalance ratios. Some earlier simulations of strong imbalanced turbulence were limited to rather idealized set ups (Maron and Goldreich 2001; Cho et al. 2002), i.e. for the initial state the results of the simulations of strong balanced turbulence were used, but the

\footnotetext{
13 Another name for imbalanced turbulence is a turbulence with nonzero cross-helicity.
} 


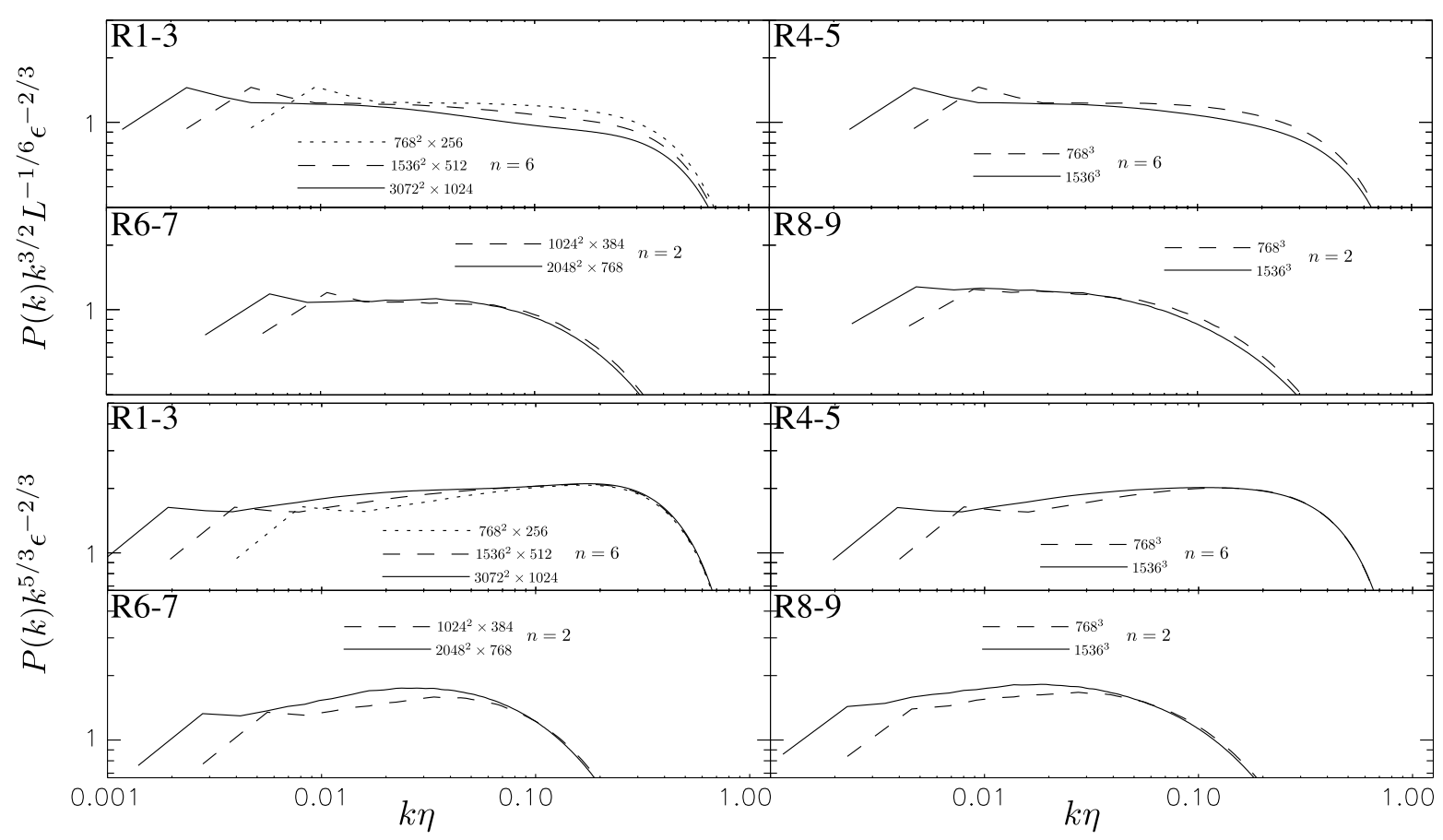

Fig. 8 Numerical convergence of spectra in 3D reduced MHD simulations. Two upper rows are used to study convergence assuming Boldyrev model and two bottom rows - assuming Goldreich-Sridhar model. $P(k)$ is so-called one-dimensional spectrum. Note that definition of dissipation scale $\eta$ depends on the model, and although this difference is tiny in hyperviscous simulations R1-5, but significant in viscous simulations R6-9. Numerical convergence require that spectra will be similar on small scales, including the dissipation scale, see, e.g. Gotoh et al. (2002). As we see from the plots, numerical convergence is absent for Boldyrev model. For Goldreich-Sridhar model the convergence is reached at the dissipation scale. Higher-resolution simulations are required to demonstrate convergence in the inertial range. Plotted with data in Beresnyak and Lazarian (2010).
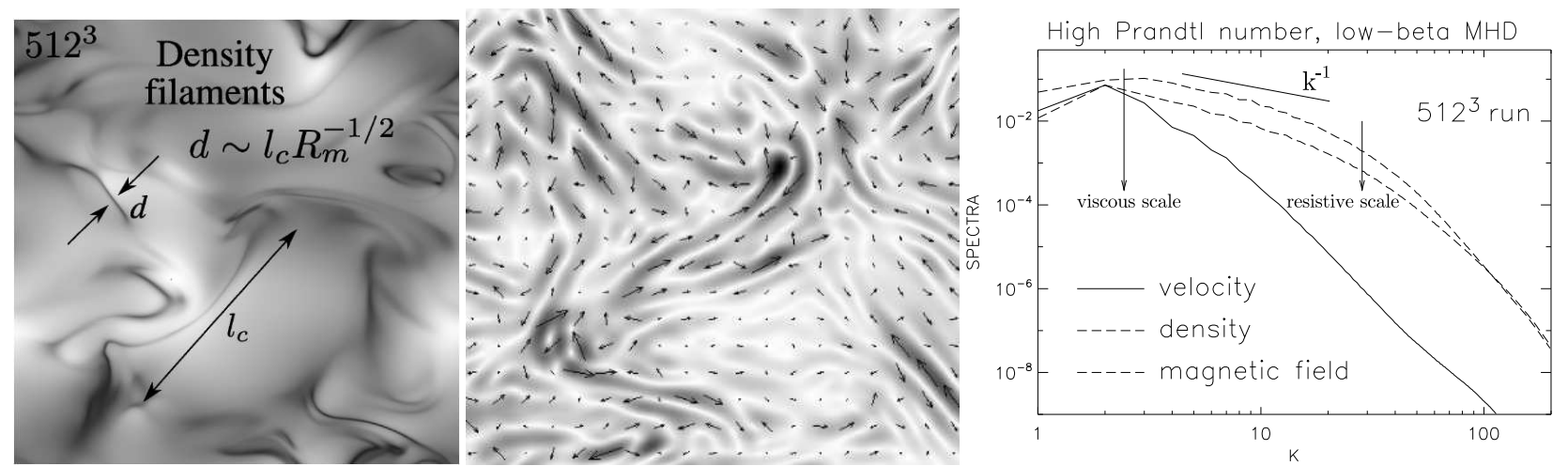

Fig. 9 Left: Filaments of density created by magnetic compression of the gas in the viscosity-damped regime of MHD turbulence. Darker regions correspond to higher density. The viscous damping scale $l_{c}$ is much larger than the current sheet thickness $d$. This creates large observed density contrasts. Center: Magnetic reversals (in the plane $\perp$ to mean $\langle\mathbf{B}\rangle$ ) that create compressions of density. Darker regions correspond to higher magnetic field. Right: Spectra of density and magnetic field are similar, while velocity is damped. The resistive scale in this regime is not $L / R m$ but $L R m^{-1 / 2}$. From Beresnyak \& Lazarian (preprint).

amplitudes of waves moving in one direction were reduced. This did not allow making definitive conclusions about properties of imbalanced turbulence. cal simulations performed so far (Beresnyak and Lazarian 2010). This model can be viewed as an extension of GS95 model into the imbalanced regime.

While the classic formulation of the GS95 critical bal-

Attempts to construct the model of stationary strong imbalanced turbulence were done in Lithwick et al. (2007); Beresnyarkenめdsezlanian ausality, is unable to describe consistently (2008); Chandran (2008); Perez and Boldyrev (2009). Below we discuss only the model in Beresnyak and Lazarian (2008) as this is the only model that agrees with numeri- the imbalanced case, Beresnyak and Lazarian (2008) proposed a new way to relate parallel and perpendicular scales of Alfvénic modes. The new condition was obtained appeal- 

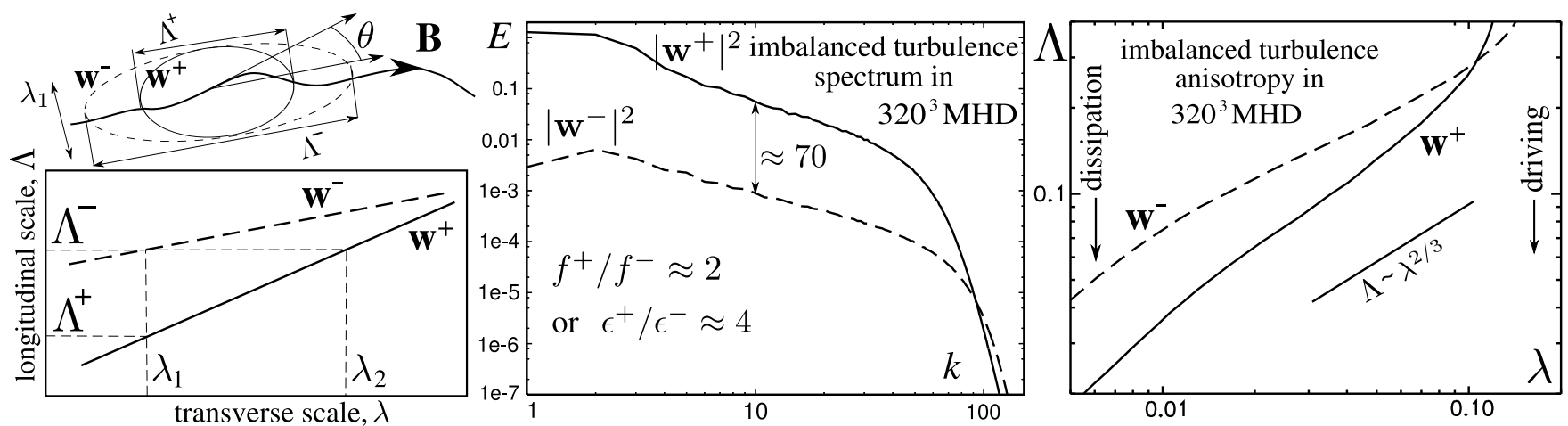

Fig. 10 Left upper: $\mathbf{w}^{+}$wavepacket, produced by cascading by $\mathbf{w}^{-}$wavepacket is aligned with respect to $\mathbf{w}^{-}$wavepacket, but disaligned with respect to the local mean field on scale $\lambda_{1}$, by the angle $\theta$. Left lower: the longitudinal scale $\Lambda$ of the wavepackets, as a function of their transverse scale, $\lambda$; Middle: the power spectrum of energies for both waves in an imbalanced forced incompressive $320^{3}$ numerical simulation. Right: the $\Lambda(\lambda)$ dependence in the same simulation, the length scales are in the units of the cube size. From Beresnyak and Lazarian (2008).

ing to the process termed "propagation balance". In the case of the balanced turbulence the "old" and "new" critical balance condition results in the same GS95 scaling. However, in the case of imbalanced turbulence the new formulation predicts smaller anisotropy for the stronger wave (which directly contradicts old causal critical balance) is consistent with simulations. The Beresnyak and Lazarian (2008) model predicts that the anisotropies of the two waves are different and this should be taken into account for calculating the scattering and acceleration arising from the particlewave interactions.

\section{Magnetic reconnection in astrophysical fluids}

\subsection{Problem of magnetic reconnection}

A magnetic field embedded in a perfectly conducting fluid is generally believed to preserves its topology for all time (Parker 1979). Although ionized astrophysical objects, like stars and galactic disks, are almost perfectly conducting, they show indications of changes in topology, "magnetic reconnection", on dynamical time scales (Parker 1970; Lovelace 1976; Priest and Forbes 2002). Reconnection can be observed directly in the solar corona (Innes et al. 1997; Yokoyama and Shib 1995; Masuda et al. 1994), but can also be inferred from the existence of large scale dynamo activity inside stellar interiors (Parker 1993; Ossendrijver 2003). Solar flares (Sturrock 1966) and $\gamma$-ray busts (Fox et al. 2005; Galama et al. 1998) are usually associated with magnetic reconnection. A lot of work in the field has concentrated on showing how reconnection can be rapid in plasmas with very small collisional
A picture of two flux tubes of different directions which get into contact in 3D space is a generic framework to describe magnetic reconnection. The upper panel of Figure 11 illustrates why reconnection is so slow in the textbook SweetParker model. Indeed, the model considers magnetic fields that are laminar and therefore the frozen-in condition for magnetic field is violated only over a thin layer dominated by plasma resistivity. The scales over which the resistive diffusion is important are microscopic and therefore the layer is very thin, i.e. $\Delta \ll L_{x}$, where $L_{x}$ is the scale at which magnetic flux tubes come into contact. The latter is of the order of the diameter of the flux tubes and typically very large for astrophysical conditions. During the process of magnetic reconnection all the plasma and the shared magnetic flux 14 arriving over an astrophysical scale $L_{x}$ should be ejected through a microscopic slot of thickness $\Delta$. As the ejection velocity of magnetized plasmas is limited by Alfvén velocity $V_{A}$, this automatically means that the velocity in the vertical direction, which is reconnection velocity, is much smaller than $V_{A}$.

We note that if magnetic reconnection is slow in some astrophysical environments, this automatically means that the results of present day numerical simulations in which the reconnection is inevitably fast due to numerical diffuhisidity do not correctly represent magnetic field dynamics in these environments. If, for instance, the reconnection were slow in collisional media this would entail the conclusion that the entire crop of interstellar, protostellar and stellar MHD calculations would be astrophysically irrelevant. To make the situation more complicated, solar flares demonstrate both periods of slow reconnection when the accumulation of magnetic flux takes place and periods of fast reconrates (Shay et al. 1998; Drake 2001; Drake et al. 2006; Daughtoneqction when the flare actually occurs. This bimodal charac2006), which substantially constrains astrophysical applications of the corresponding reconnection models. The disregard of pre-existing turbulence is another shortcoming of these models.
${ }^{14}$ Figure 11 presents a cross section of the 3D reconnection layer. A guide field is present in the generic 3D configurations of reconnecting magnetic flux tubes. 
ter is another property that a successful model of reconnection should address. and particle acceleration throughout the universe (de Gouveia Dal Pino and 2003, 2005; Lazarian 2005; Drake et al. 2006).

In this section we focus our attention on the reconnection that takes place in turbulent astrophysical fluids. The reconnection in such environments was described in Lazarian and Vishniad Rate of reconnection (1999). The latter model identified magnetic field wandering as the key process that induces fast, i.e. independent of fluid resistivity, magnetic reconnection. The predicted dependences of magnetic reconnection on the properties of turbulence, i.e. on the intensity and injection scale of turbulence, have been successfully tested in Kowal et al. (2009. 2012). The deep consequence of the model is the violation of the magnetic flux freezing in turbulent fluids (e.g. the explicit claim of this in Vishniac and Lazarian 1999). The connection of the LV99 model with more recent ideas on the properties of magnetic field in turbulent plasmas are analyzed in Evink et al. (2011).

While the discussion of possible ways how turbulence can enhance reconnection rates was not unprecedented, the LV99 model was radically different from what was proposed earlier. For instance, the closest to the spirit of LV99, are two papers by Matthaeus and Lamkin (1985, 1986), where the authors performed 2D numerical simulations of turbulence and provided arguments in favor of magnetic reconnection getting fast. However, the physics of the processes that they considered was very different from that in LV99. For instance, the key process of field wandering of the LV99 model has not been considered by Matthaeus and Lamkin (1985, 1986). On the contrary, the components of their approach, e.g. X-point and possible effects of heating and compressibility are not ingredients of the LV99 model. Other papers, e.g. Speisen (1970) and Jacobson and Moses (1984) are even more distant in terms of the effects that they explored, namely, they studied the changes of the microscopic properties of the plasma induced by turbulence and considered how these changes can accelerate magnetic reconnection. At the same time, LV99 shows that the microscopic plasma properties are irrelevant for their model of reconnection.

Below (\$5) we present numerical evidence, based on three dimensional simulations, that reconnection in a turbulent fluid occurs at a speed comparable to the rms velocity of the turbulence, regardless of either the value of the resistivity or degree of collisionality. In particular, this is true for turbulent pressures much weaker than the magnetic field pressure so that the magnetic field lines are only slightly bent by the turbulence. These results are consistent with the proposal by LV99 that reconnection is controlled by the stochastic diffusion of magnetic field lines, which produces a broad outflow of plasma from the reconnection zone. This implies that reconnection in a turbulent fluid typically takes place in approximately a single eddy turnover time, with broad implications for dynamo activity (Parker 1970, 1993; Stix 2000)
Astrophysical plasmas are often highly ionized and highly magnetized (Parker 1970). The evolution of the magnetic field in a highly conducting fluid can be described by a simple version of the induction equation

$\frac{\partial \mathbf{B}}{\partial t}=\nabla \times(\mathbf{v} \times \mathbf{B}-\eta \nabla \times \mathbf{B})$,

where $\mathbf{B}$ is the magnetic field, $\mathbf{v}$ is the velocity field, and $\eta$ is the resistivity coefficient. Under most circumstances this is adequate for discussing the evolution of magnetic field in an astrophysical plasma. When the dissipative term on the right hand side is small, as is implied by simple dimensional estimates, the magnetic flux through any fluid element is constant in time and the field topology is an invariant of motion. On the other hand, reconnection is observed in the solar corona and chromosphere (Innes et al. 1997; Yokoyama and Shibata 1995; Masuda et al. 1994; Ciaravella and Ray 2008), its presence is required to explain dynamo action in stars and galactic disks (Parker 1970, 1993), and the violent relaxation of magnetic fields following a change in topology is a prime candidate for the acceleration of high energy particles (de Gouveia Dal Pino and Lazarian 2003, 2005; Lazarian 2005; Drake et al. 2006; Lazarian 2009; Drake et al. 2010) in the universe. Quantitative general estimates for the speed of reconnection start with two adjacent volumes with different large scale magnetic field directions (Sweet 1958; Parker 1957).

The speed of reconnection, i.e. the speed at which inflowing magnetic field is annihilated by Ohmic dissipation, is roughly $\eta / \Delta$, where $\Delta$ is the width of the transition zone (see Figure 11). Since the entrained plasma follows the local field lines, and exits through the edges of the current sheet at roughly the Alfvén speed, $V_{A}$, the resulting reconnection speed is a tiny fraction of the Alfvén speed, $V_{A} \equiv$ $B /(4 \pi \rho)^{1 / 2}$ where $L$ is the length of the current sheet. When the current sheet is long and the reconnection speed is slow this is referred to as Sweet-Parker reconnection. Observations require a speed close to $V_{A}$, so this expression implies that $L \sim \Delta$, i.e. that the magnetic field lines reconnect in an "X point".

The first model with a stable $\mathrm{X}$ point was proposed by Petschek (1964). In this case the reconnection speed may have little or no dependence on the resistivity. The $\mathrm{X}$ point configuration is known to be unstable to collapse into a sheet in the MHD regime (see Biskamp 1996), but in a collisionless plasma it can be maintained through coupling to a dispersive plasma mode (Sturrock 1966). This leads to fast reconnection, but with important limitations. This process has 
a limited astrophysical applicability as it cannot be impor-

$L_{x}$ tant for most phases of the interstellar medium (see Draine and Lazarian
1998, for a list of the idealized phases), not to speak about
dense plasmas, such as stellar interiors and the denser parts netic fields are not wound around each other, producing a saddle shaped current sheet. In that case the energy required to open up an $\mathrm{X}$ point is prohibitive. The saddle current sheet is generic for not parallel flux tubes trying to pass through each other. If such a passage is seriously constrained, the magnetized highly conducting astrophysical fluids should behave more like Jello rather than normal fluid 15 .

Finally, the traditional reconnection setup does not include ubiquitous astrophysical turbulence ${ }^{16}$ (see Armstrong et al. 1995; Elmegreen and Scalo 2004; McKee and Ostriker 2007; Lazarian 2009; Chepurnov and Lazarian 2010). Fortunately, this approach provides another way of accelerating reconnection. Indeed, an alternative approach is to consider ways to decouple the width of the plasma outflow region from $\Delta$. The plasma is constrained to move along magnetic field lines, but not necessarily in the direction of the mean magnetic field. In a turbulent medium the two are decoupled, and fluid elements that have some small initial separation will be separated by a large eddy scale or more after moving the length of the current sheet. As long as this separation is larger than the width of the current sheet, the result will not depend on $\eta$.

LV99 introduced a model that included the effects of magnetic field line wandering (see Figure 11). The model relies on the nature of three-dimensional magnetic field wandering in turbulence. The effects of compressibility and heating, which were thought to be important in the earlier studies (see, for example, the discussion in Matthaeus and Lamkin 1985, 1986), do not play the role for the LV99 model either. The model is applicable to any weakly perturbed magnetized fluid, irrespectively, of the degree of plasma being collisional or collisionless (cf. Shay et al. 1998).

Two effects are the most important for understanding of the nature of reconnection in LV99. First of all, in three dimensions bundles of magnetic field lines can enter the reconnection region and reconnection there independently (see Figure 11), which is in contrast to two dimensional picture where in Sweet-Parker reconnection the process is artificially constrained. Then, the nature of magnetic field stochasticity and therefore magnetic field wandering (which determines the outflow thickness, as illustrated in Figure 11)

\footnotetext{
15 The idea of felt or Jello-type structure of magnetic fields in interstellar medium was advocated by Don Cox (private communication).

16 The set ups where instabilities play important role include Shimizu et al. (2009b 2009a). For sufficiently large resolution of simulations those set-ups are expected to demonstrate turbulence. Turbulence initiation is also expected in the presence of plasmoid ejection (Shibata and Tanuma 2001). Numerical viscosity constrains our ability to sustain turbulence via reconnection, however.
}

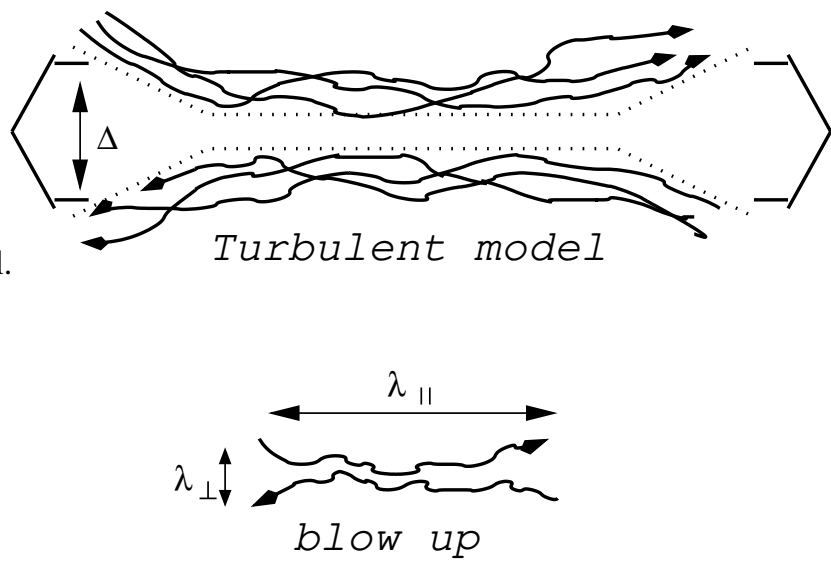

Fig. 11 Upper plot: Sweet-Parker model of reconnection. The outflow is limited by a thin slot $\Delta$, which is determined by Ohmic diffusivity. The other scale is an astrophysical scale $L \gg \Delta$. Middle plot: Reconnection of weakly stochastic magnetic field according to LV99. The model that accounts for the stochasticity of magnetic field lines. The outflow is limited by the diffusion of magnetic field lines, which depends on field line stochasticity. Low plot: An individual small scale reconnection region. The reconnection over small patches of magnetic field determines the local reconnection rate. The global reconnection rate is substantially larger as many independent patches come together. From Lazarian et al. (2004).

is very different in 2D and the real 3D world (LV99). In other words, removing artificial constraints on the dimensionality of the reconnection region and the magnetic field being absolutely straight, LV99 explores the real-world astrophysical reconnection.

The scaling relations for Alfvénic turbulence allow us to calculate the rate of magnetic field spreading. This is the effect known to be important for the cosmic ray propagation Jokipii (1973) and LV99 quantified it for the case of GS95 turbulence 17 . A bundle of field lines confined within a region of width $y$ at some particular point will spread out perpendicular to the mean magnetic field direction as one moves in either direction following the local magnetic field lines. The rate of field line diffusion is given approximately by

$\frac{d\left\langle y^{2}\right\rangle}{d x} \sim \frac{\left\langle y^{2}\right\rangle}{\lambda_{\|}}$

\footnotetext{
17 This treatment was used later for dealing with different problems, from heat propagation in plasmas Narayan and Medvedev (2001); Lazarian and Pogosyan (2006) to cosmic ray propagation Yan and Lazarian (2008).
} 
where $\lambda_{\|}^{-1} \approx \ell_{\|}^{-1}, \ell_{\|}$is the parallel scale chosen so that the corresponding vertical scale, $\ell_{\perp}$, is $\sim\left\langle y^{2}\right\rangle^{1 / 2}$, and $x$ is the distance along an axis parallel to the mean magnetic field. Therefore, using equation (3) one gets

$\frac{d\left\langle y^{2}\right\rangle}{d x} \sim L_{i}\left(\frac{\left\langle y^{2}\right\rangle}{L_{i}^{2}}\right)^{2 / 3}\left(\frac{u_{L}}{v_{A}}\right)^{4 / 3}$

where we have substituted $\left\langle y^{2}\right\rangle^{1 / 2}$ for $\ell_{\perp}$. This expression for the diffusion coefficient will only apply when $y$ is small enough for us to use the strong turbulence scaling relations, or in other words when $\left\langle y^{2}\right\rangle<L_{i}^{2}\left(u_{L} / v_{A}\right)^{4}$. Larger bundles will diffuse at a maximum rate of $L_{i}\left(u_{L} / v_{A}\right)^{4}$. For $\left\langle y^{2}\right\rangle$ small equation (9) implies that a given field line will wander perpendicular to the mean field line direction by an average amount

$\left\langle y^{2}\right\rangle^{1 / 2} \approx \frac{x^{3 / 2}}{L_{i}^{1 / 2}}\left(\frac{u_{L}}{v_{A}}\right)^{2}$

in a distance $x$. The fact that the rms perpendicular displacement grows faster than $x$ is significant. It implies that if we consider a reconnection zone, a given magnetic flux element that wanders out of the zone has only a small probability of wandering back into it.

The advantage of the classical Sweet-Parker scheme of reconnection is that it naturally follows from the idea of Ohmic diffusion. Indeed, mass conservation requires that the inflow of matter through the scale of the contact region $L_{x}$ be equal to the outflow of matter through the diffusion layer $\Delta$, i.e.

$v_{\text {rec }}=v_{A} \frac{\Delta}{L_{x}}$.

The mean-square vertical distance that a magnetic fieldline can diffuse by resistivity in time $t$ is

$\left\langle y^{2}(t)\right\rangle \sim \lambda t$.

The field lines are advected out of the sides of the reconnection layer of length $L_{x}$ at a velocity of order $v_{A}$. Thus, the time that the lines can spend in the resistive layer is the Alfvén crossing time $t_{A}=L_{x} / v_{A}$. Thus, field lines can only be merged that are separated by a distance

$\Delta=\sqrt{\left\langle y^{2}\left(t_{A}\right)\right\rangle} \sim \sqrt{\lambda t_{A}}=L_{x} / \sqrt{S}$,

where $S$ is Lundquist number. Combining Eqs. (11) and (13) one gets the famous Sweet-Parker reconnection rate, $v_{\text {rec }}=$ $v_{A} / \sqrt{S}$.

In LV99 magnetic field wandering determines the scale of the outflow $\Delta$ (see Figure 11). Using expressions from the earlier section one can obtain (LV99):

$V_{r e c}<v_{A} \min \left[\left(\frac{L_{x}}{L_{i}}\right)^{1 / 2},\left(\frac{L_{i}}{L_{x}}\right)^{1 / 2}\right]\left(u_{L} / V_{A}\right)^{2}$.

This limit on the reconnection speed is fast, both in the sense that it does not depend on the resistivity, and in the sense that it represents a large fraction of the Alfvén speed. To prove that Eq. (14) indeed constitutes the reconnection rate LV99 goes through a thorough job of considering all other possible bottlenecks for the reconnection and shows that they provide higher reconnection speed.

Below we provide a new derivation of the LV99 reconnection rates which makes apparent that the LV99 model is a natural generalization of the laminar Sweet-Parker model to flows with background turbulence. The new argument in Eyink et al. (2011) is based on the concept of Richardson diffusion. It is known in hydrodynamic turbulence that the combination of small scale diffusion and large scale shear gives rise to Richardson diffusion, where the mean square separation between two particles grows as $t^{3}$ once the rms separation exceeds the viscous damping scale. A similar phenomenon occurs in MHD turbulence. In both cases the separation at late times is independent of the microscopic transport coefficients. Although the plasma is constrained to move along magnetic field lines, the combination of turbulence and ohmic dissipation produces a macroscopic region of points that are "downstream" from the same initial volume, even in the limit of vanishing resistivity.

Richardson diffusion (see Kupiainen 2003) implies the mean squared separation of particles $\left\langle\left|x_{1}(t)-x_{2}(t)\right|^{2}\right\rangle \approx \varepsilon t^{3}$, where $t$ is time, $\varepsilon$ is the energy cascading rate and $\langle\cdot\rangle$ denotes an ensemble averaging. For subAlfvenic turbulence $\varepsilon \approx u_{L}^{4} /\left(v_{A} L_{i}\right)$ (see LV99) and therefore analogously to Eq. (13) one can write

$\Delta \approx \sqrt{\varepsilon t_{A}^{3}} \approx L\left(L / L_{i}\right)^{1 / 2}\left(u_{L} / V_{A}\right)^{2}$

where it is assumed that $L<L_{i}$. Combining Eqs. (11) and (15) one gets

$V_{\text {rec }, L V 99} \approx v_{A}\left(L / L_{i}\right)^{1 / 2}\left(u_{L} / V_{A}\right)^{2}$.

in the limit of $L<L_{i}$. Analogous considerations allow to recover the LV99 expression for $L>L_{i}$, which differs from Eq. (16) by the change of the power $1 / 2$ to $-1 / 2$. These results coincide with those given by Eq. (14).

It is important to stress that Richardson diffusion ultimately leads to diffusion over the entire width of large scale eddies once the plasma has moved the length of one such eddy. The precise scaling exponents for the turbulent cascade does not affect this result, and all of the alternative scalings considered in LV99 yield the same behavior.

Other forms of reconnection rate are also useful. For instance, one can take into account that the velocity of the transition to the strong turbulent regime is $u_{\text {turb,strong }} \sim v_{A} M_{A}^{2}$. Then

$V_{\text {rec }} \approx u_{\text {turb }, \text { strong }}\left(l / L_{i}\right)^{1 / 2}$,

shows that the reconnection rate scales with the velocity of the strong turbulence cascade. Similarly, it is useful to rewrite this expression in terms of the power injection rate 
$P_{i n j}$. As the perturbations on the injection scale of turbulence are assumed to have velocities $u_{l}<V_{A}$, the turbulence is weak at large scales. Therefore, the relation between the power and the injection velocities are different from the usual Kolmogorov estimate, namely, in the case of the weak turbulence $P \sim u_{l}^{4} /\left(l V_{A}\right)$ (LV99). Thus we get,

$V_{\text {rec }} \approx\left(\frac{P_{i n j}}{L V_{A}}\right)^{1 / 2} l$,

where $l$ is the length of the turbulent eddies parallel to the large scale magnetic field lines as well as the injection scale.

If turbulence is superAlfvénic, LV99 predicts that the diffusion of magnetic field happens with the turbulent velocity. This is possible as the subAlfvénic reconnection that happens below the scale $l_{A}$ given by Eq. (6) does not present the bottleneck for the reconnection at the larger scales.

The LV99 reconnection velocity (e.g. Eq. (18) ) does not depend on resistivity or plasma effects. Therefore for sufficiently high level of turbulence we expect both collisionless and collisional fluids to reconnect at the same rate.

\subsection{Model of flares}

If turbulence can drive reconnection, which in turn transforms magnetic energy into kinetic energy, then it seems appropriate to wonder if the process can be self-sustaining. That is, given a very small level of ambient turbulence, how likely is it that reconnection will speed up as it progresses, without any further input from the surrounding medium? The corresponding problem was in LV99. Below we follow simple arguments provided in Lazarian and Vishniac (2009), which clarify the related physics by presenting an idealized model of a reconnection flare.

Let's consider a reconnection region of length $L$ and thickness $\Delta$. The thickness is determined by the diffusion of field lines, which is in turn determined by the strength of the turbulence in the volume. Reconnection will allow the magnetic field to relax, creating a bulk flow. However, since stochastic reconnection is expected to proceed unevenly, with large variations in the current sheet, we can expect that some unknown fraction of this energy will be deposited inhomogeneously, generating waves and adding energy to the local turbulent cascade. We take the plasma density to be approximately uniform so that the Alfvén speed and the magnetic field strength are interchangeable. The nonlinear dissipation rate for waves is

$\tau_{\text {nonlinear }}^{-1} \sim \max \left[\frac{k_{\perp}^{2} v_{\text {wave }}^{2}}{k_{\|} V_{A}}, k_{\perp}^{2} v_{\text {turb }} \lambda_{\perp, t u r b}\right]$,

where the first rate is the self-interaction rate for the waves and the second is the dissipation rate by the ambient turbulence (see Beresnyak and Lazarian 2008). The important point here is that $k_{\perp}$ for the waves falls somewhere in the inertial range of the strong turbulence. Eddies at that wavenumber will disrupt the waves in one eddy turnover time, which is necessarily less than $L / V_{A}$. The bulk of the wave energy will go into the tubulent cascade before escaping from the reconnection zone. (This zone will radiate waves, for the same reason that turbulence in general radiates waves, but it will not significantly impact that energy budget of the reconnection region.)

We can therefore simplify our model for the energy budget in the reconnection zone by assuming that some fraction $\varepsilon$ of the energy liberated by stochastic reconnection is fed into the local turbulent cascade. The evolution of the turbulent energy density per area is

$\frac{d}{d t}\left(\Delta v_{\text {turb }}^{2}\right)=\varepsilon V_{A}^{2} V_{\text {rec }}-v_{\text {turb }}^{2} \Delta \frac{V_{A}}{L}$,

where the loss term covers both the local dissipation of turbulent energy, and its advection out of the reconnection zone. Since $V_{\text {rec }} \sim v_{\text {turb }}$ and $\Delta \sim L\left(v_{\text {turb }} / V_{A}\right)$, we can rewrite this by defining $\mathscr{M}_{A} \equiv v_{\text {turb }} / V_{A}$ and $\tau \equiv L / V_{A}$ so that

$$
\frac{d}{d \tau} \mathscr{M}_{A}^{3} \approx \varepsilon \mathscr{M}_{A}-\mathscr{M}_{A}^{3} \text {. }
$$

If $\varepsilon$ is a constant then

$v_{\text {turb }} \approx V_{A} \varepsilon^{1 / 2}\left[1-\left(1-\frac{\mathscr{M}_{A}^{2}}{\varepsilon}\right) e^{-2 \tau / 3}\right]^{1 / 2}$.

This implies that the reconnection rate rises to $\varepsilon^{1 / 2} V_{A}$ is a time comparable to the ejection time from the reconnection region $\left(\sim L / V_{A}\right)$. Given that reconnection events in the solar corona seem to be episodic, with longer periods of quiescence, this implies that either $\varepsilon$ is very small, for example dependent on the ratio of the thickness of the current sheet to $\Delta$, or is a steep function of $\mathscr{M}_{A}$. If it scales as $\mathscr{M}_{A}$ to some power greater than two then initial conditions dominate the early time evolution.

An alternative route by which stochastic reconnection might be self-sustaining would be in the context of a series of topological knots in the magnetic field, each of which is undergoing reconnection. Now the problem is sensitive to geometry. Let's assume that as each knot undergoes reconnection it releases a characteristic energy into a volume which has the same linear dimension as the distance to the next knot. The density of the energy input into this volume is roughly $\varepsilon V_{A}^{2} v_{\text {turb }} / L$, where $\varepsilon$ is the efficiency with which the magnetic energy is transformed into turbulent energy. We have

$\varepsilon V_{A}^{2} \frac{v_{t u r b}}{L} \sim \frac{v^{\prime 3}}{L_{k}}$

where $L_{k}$ is the distance between knots and $v^{\prime}$ is the turbulent velocity created by the reconnection of the first knot. This process will proceed explosively if $v^{\prime}>v_{\text {turb }}$ or

$V_{A}^{2} L_{k} \varepsilon>v_{\text {turb }}^{2} L$. 
This condition is almost trivial to fulfill. The bulk motions created by reconnection will unavoidably generate significant turbulence as they interact with their surrounding, so $\varepsilon$ should be of order unity. Moreover the length of any current sheet should be at most comparable to the distance to the nearest distinct magnetic knot. The implication is that each magnetic reconnection event will set off its neighbors, boosting their reconnection rates from $v_{t u r b}$, set by the environment, to $\varepsilon^{1 / 2} V_{A}\left(L_{k} / L\right)^{1 / 2}$ (as long as this is less than $V_{A}$ ). The process will take a time comparable to $L / v_{\text {turb }}$ to begin, but once initiated will propagate through the medium with a speed comparable to speed of reconnection in the individual knots. In a more realistic situation, the net effect will be a kind of modified sandpile model for magnetic reconnection in the solar corona and chromosphere. As the density of knots increases, and the energy available through magnetic reconnection increases, the chance of a successfully propagating reconnection front will increase.

The flare of reconnection presents a reconnection instability. In the absence of external turbulence the original outflow, e.g. originated through tearing instability (see Loureiro et al. 2007, Bhattacharjee et al. 2009) gets turbulent and triggers the mechanism described above. This shows that the tearing and turbulent mechanisms may be complementary 18

\section{Testing of LV99 model}

Here we describe the results of a series of three dimensional numerical simulations aimed at adding turbulence to the simplest reconnection scenario and testing equation (18). We divide the domain in the middle into two equal regions along the vertical direction (along the $\mathrm{Y}$ axis in our case) with equal magnetic field strength, but opposite sign of its X component. In addition, a constant guide field is added in the $\mathrm{Z}$ direction. The domain is periodic along the guide field (i.e. the $\mathrm{Z}$ axis) and open in the other directions (the $\mathrm{X}$ and $\mathrm{Y}$ axis). The external gas pressure is uniform and the magnetic fields at the top and bottom of the box are taken to be the specified external fields plus small perturbations to allow for outgoing waves. The grid size in the simulations varied from $256 \times 512 \times 256$ to $512 \times 1028 \times 512$ so that the top and bottom of the box are far away from the current sheet and the region of driven turbulence around it. At the sides of the box where outflow is expected the derivatives of the dynamical variables are set to zero. A complete description of the numerical methodology can be found in Kowal et al. (2009). All simulations are allowed to evolve for seven Alfvén crossing times without turbulent forcing. During this time they

\footnotetext{
18 When turbulence develops the LV99 mechanism can provide much faster reconnection compared to tearing and tearing may become a subdominant process. In fact, emerging turbulence may suppress the tearing instability.
}

develop the expected Sweet-Parker current sheet configuration with slow reconnection. Subsequently the isotropic turbulent forcing is turned on inside a volume centered in the midplane (in the XZ plane) of the simulation box and extending outwards by a quarter of the box size. The turbulence reaches its full amplitude around eight crossing times and is stationary thereafter.

The speed of reconnection in three dimensions can be hard to define without explicit evaluation of the magnetic field topology. However, in this simple case we can define it as the rate at which the $x$ component of the magnetic field disappears. More precisely, we consider a yz slice of the simulation, passing through the center of the box. The rate of change of the area integral of $-B_{x}-$ is its flux across the boundaries of the box minus the rate at which flux is annihilated through reconnection (see more discussion in Kowal et al. 2009)

$\partial_{t}\left(\int\left|B_{x}\right| d z d y\right)=\oint \operatorname{sign}\left(B_{x}\right) \mathbf{E} d \mathbf{l}-2 V_{r e c} B_{x, e x t} L_{z}$

where electric field is $\mathbf{E}=\mathbf{v} \times \mathbf{B}-\eta \mathbf{j}, B_{x, \text { ext }}$ is the absolute value of $B_{x}$ far from the current sheet and $L_{z}$ is the width of the box in the $z$ direction. This follows from the induction equation under the assumption that the turbulence is weak to lead to local field reversals and that the stresses at the boundaries are weak to produce significant field bending there. In other words, fields in the $x$ direction are advected through the top and bottom of the box, and disappear only through reconnection. Since periodic boundary conditions are adopted in the $z$ direction the boundary integral on the right hand side is only taken over the top and bottom of the box. By design this definition includes contributions to the reconnection speed from contracting loops, where Ohmic reconnection has occurred elsewhere in the box and $\left|B_{x}\right|$ decreases as the end of a reconnected loop is pulled through the plane of integration. It is worth noting that this estimate is roughly consistent with simply measuring the average influx of magnetic field lines through the top and bottom of the computational box and equating the mean inflow velocity with the reconnection speed. Following equation (25) we can evaluate the reconnection speed for varying strengths and scales of turbulence and varying resistivity.

In Figure 13 we see the results for varying amounts of input power, for fixed resistivity and injection scale as well as for the case of no turbulence at all. The line drawn through the simulation points is for the predicted scaling with the square root of the input power. The agreement between equation (18) and Figure 13 is encouraging but does not address the most important aspect of stochastic reconnection, i.e. its insensitivity to $\eta$.

In Figure 14 we plot the results for fixed input power and scale, while varying the background resistivity. In this case $\eta$ is taken to be uniform, except near the edges of the computational grid where it falls to zero over five grid points. 

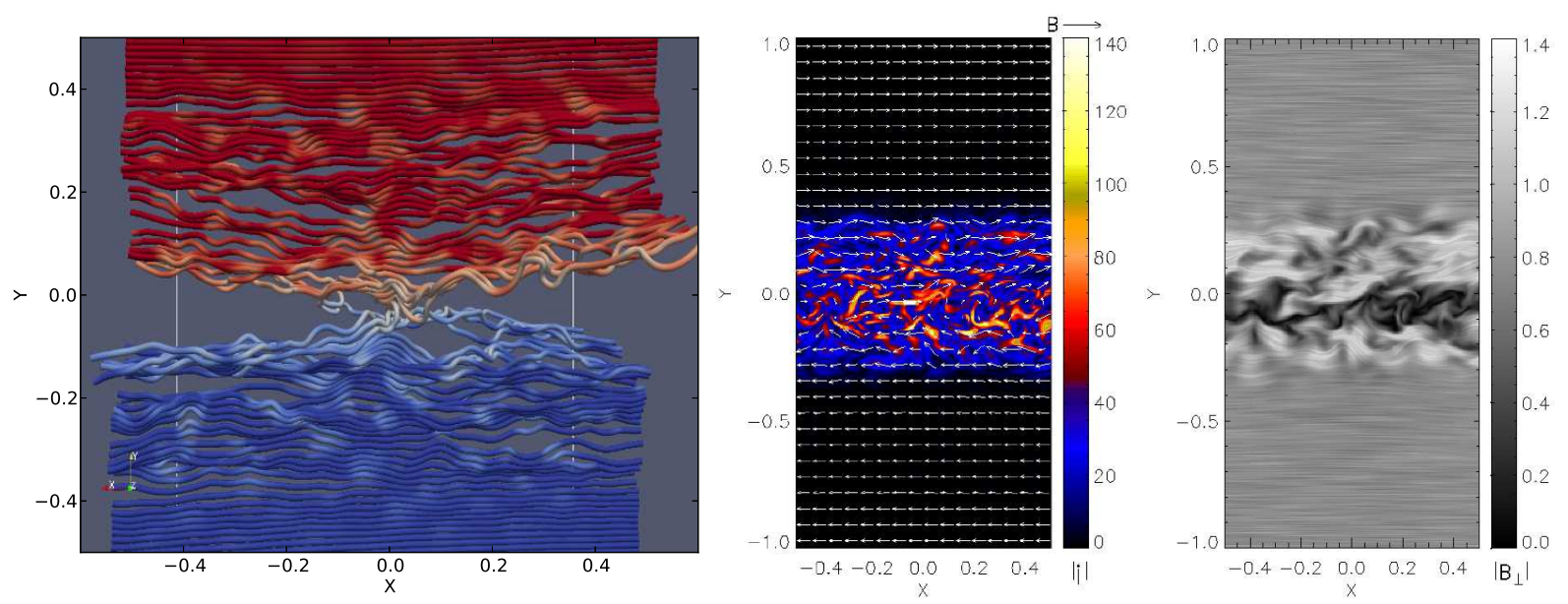

Fig. 12 Visualization of reconnection simulations in Kowal et al. 2012). Left panel: Magnetic field in the reconnection region. Large perturbations of magnetic field lines arise from reconnection rather than driving; the latter is subAlfvénic. The color corresponds to the direction of magnetic lines. Central panel: Current intensity and magnetic field configuration during stochastic reconnection. We show a slice through the middle of the computational box in the xy plane after twelve dynamical times for a typical run. The guide field is perpendicular to the page. The intensity and direction of the magnetic field is represented by the length and direction of the arrows. The color bar gives the intensity of the current. The reversal in $B_{x}$ is confined to the vicinity of $\mathrm{y}=0$ but the current sheet is strongly disordered with features that extend far from the zone of reversal. Right panel: Representation of the magnetic field in the reconnection zone with textures.

This was done to eliminate edge effects for large values of the resistivity. We see from the Figure 14 that the points for laminar reconnection scale as $\sqrt{\eta}$, the expected scaling for Sweet-Parker reconnection. In contrast, the points for reconnection in a turbulent medium do not depend on the resistivity at all. In summary, we have tested the model of stochastic reconnection in a simple geometry meant to approximate the circumstances of generic magnetic reconnection in the universe. Our results are consistent with the mechanism described by LV99. The implication is that turbulent fluids in the universe including the interstellar medium, the convection zones of stars, and accretion disks, have reconnection speeds close to the local turbulent velocity, regardless of the local value of resistivity. Magnetic fields in turbulent fluids can change their topology on a dynamical time scale.

Figure 15 shows results of numerical experiments in which the dependence of the reconnection on the anomalous resistivity was studied. The anomalous resistivity increases effective resistivity for high current densities. It is frequently used as a proxy for plasma effects, e.g. collisionless effects in reconnection. While this type of resitistivity enhances the local speed of individual reconnection events, the study in Kowal et al. (2009) testifies that the total reconnection rate does not change.

Any numerical study has to address the issue of the possible numerical effects on the results. We show the dependence of the reconnection rate on the numerical resolution in Figure 16. The reconnection rate increases with the increase of the resolution, which testifies that the fast reconnection is not due to numerical effects. Indeed, higher numerical reconnection is expected for lower resolution simulations.
Finally, it is important to give a few words in relation to our turbulence driving. We drive our turbulence solenoidally to minimize the effects of compression, which does not play a role in LV99 model. The turbulence driven in the volume around the reconnection layer corresponds to the case of astrophysical turbulence, which is also volume-driven. On the contrary, the case of the turbulence driven at the box boundaries would produce spatially inhomogeneous imbalanced turbulence for which we do not have analytical predictions (see discussion of such turbulence in Beresnyak and Lazarian 2009). We stress, that it is not the shear size of our numerical simulations, but the correspondence of the observed scalings to those predicted in LV99 that allows us to claim that we proved that the $3 \mathrm{D}$ reconnection is fast in the presence of turbulence.

\section{Turbulence and cosmic rays acceleration}

\subsection{Shock acceleration in the presence of turbulence}

Cosmic rays (CRs), relativistic charged particles with energies $10^{8}-10^{22} \mathrm{eV}$, constitute an essential part of astrophysical systems (see Schlickeiser 2003). The origin of CRs has been a subject of debate from the beginning of research in the field (Ginzburg and Syrovatskii 1964). By now, it is accepted that galactic CRs at least up to the "knee" in the spectrum, just above $10^{15} \mathrm{eV}$, are most likely generated primarily by strong supernova shocks. However, it is easy to show that typical interstellar upstream magnetic field of around $5 \mu G$ is too weak to provide the return of the cosmic rays with 

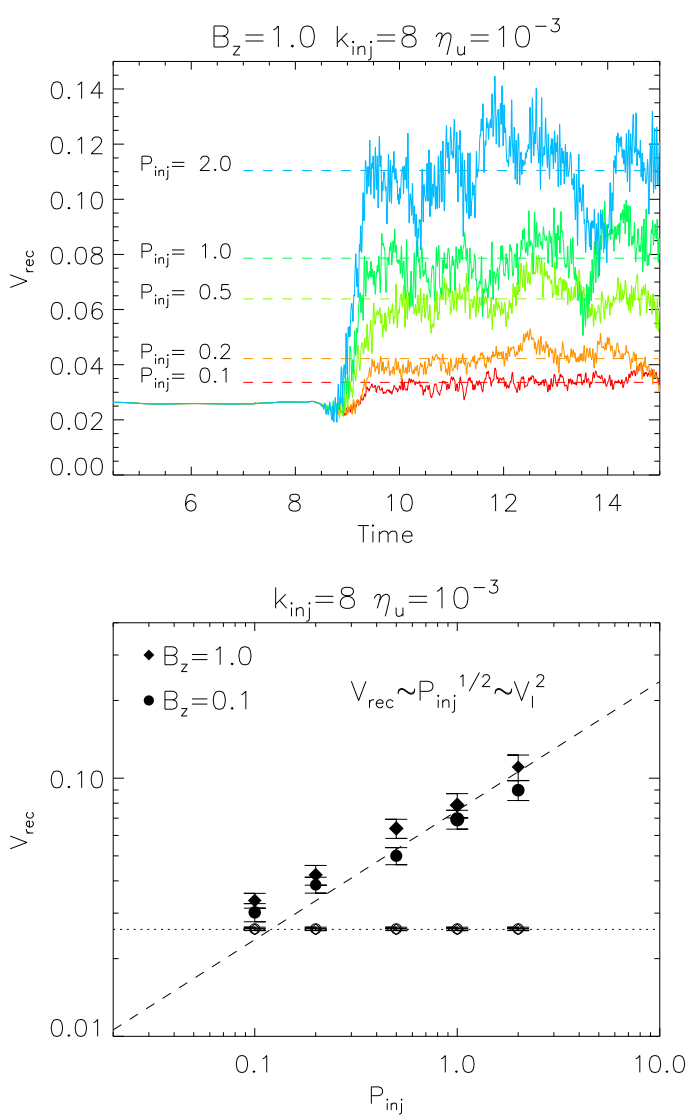

Fig. 13 Reconnection speed versus input power $P_{i n j}$ for the driven turbulence. Upper plot: Variations of reconnection speed in time for different levels of turbulent driving. Lower Plot: Reconnection speed, plotted against the input power for an injection wavenumber equal to 8 (i.e. a wavelength equal to one eighth of the box size) and a resistivity $v_{u}$. The dashed line is a fit to the predicted dependence of $P^{1 / 2}$. The horizontal line shows the laminar reconnection rates for each of the simulations before the turbulent forcing started. Here the uncertainty in the time averages are indicated by the size of the symbols and the variances are shown by the error bars. From Kowal et al. (2009).

energies $\sim 10^{15} \mathrm{GeV}$ back to the shock to continue the acceleration.

To overcome the problem it is important to increase the value of the magnetic field both in the preshock and postshock regions. The latter problem has a straightforward solution, as postshock gas is known to be turbulent. Turbulence can amplify the magnetic field (see Kazantsev 1968; Kulsrud and Anderson 1992; Cho et al. 2009; Beresnyak et al. 2011) and therefore an enhanced magnetic field in the postshock region is expected (see Giacalone and Jokipii 2007). A more challenging issue is to have the magnetic field amplified in preshock plasmas. One known way is to appeal to the streaming instability. The latter evolves to the nonlinear stage with $\delta B \gg B_{0}$ making the original classical treatment of the instability not applicable. What happens in the nonlinear regime has been discussed a lot in recent years (e.g. Blasi and Amato 2008; Lucek and Bell 2000; Diamond and

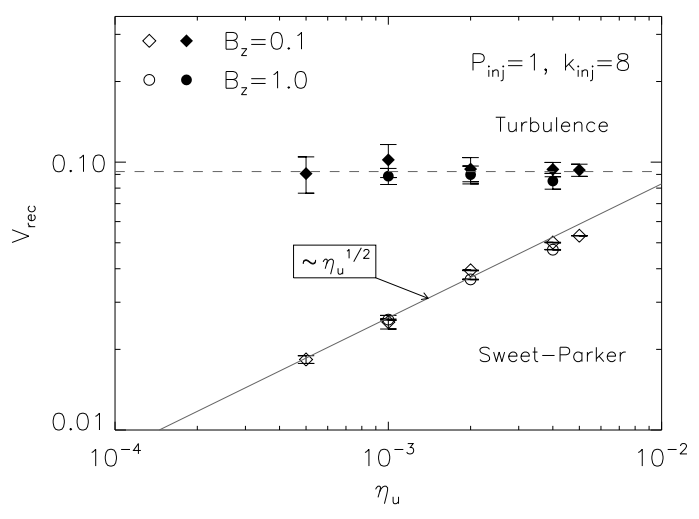

Fig. 14 Reconnection speed versus resistivity. We show the reconnection speed plotted against the uniform resistivity of the simulation for an injection wavenumber of 8 and an injected power of one. We include both the laminar reconnection speeds, using the hollow symbols, fit to the expected dependence of $\eta_{u}$, and the stochastic reconnection speeds, using the filled symbols. As before the symbol sizes indicate the uncertainty in the average reconnection speeds and the error bars indicate the variance. $B_{x}=1$ and simulations with large, $B_{z}=1$, and small, $B_{z}=0.1$, guide fields are shown. From Kowal et al. (2009).

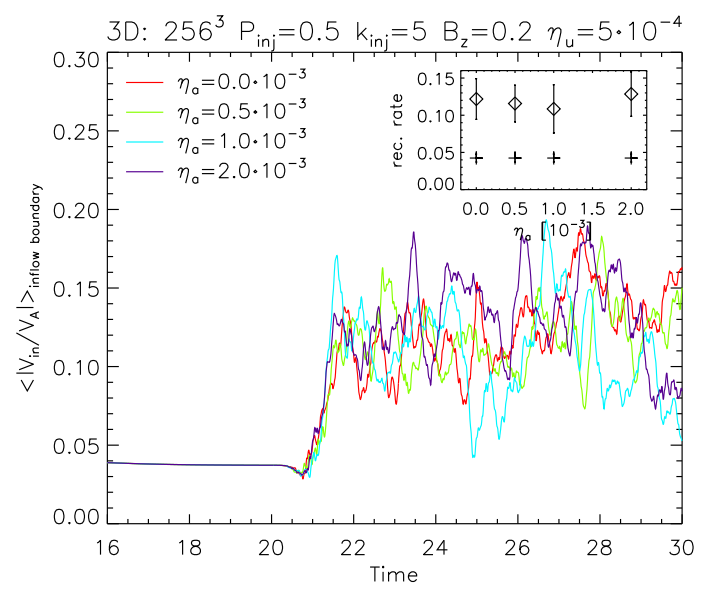

Fig. 15 Effect of anomalous resistivity on the reconnection speed. No dependence is observed. From Kowal et al. (2009).

2007; Zirakashvili et al. 2008; Riquelme and Spitkovsky 2009) with many researchers pointing out that the instability may become much suppressed. In addition, even at its linear regime the instability is likely to be suppressed in the presence of ambient turbulence (Yan and Lazarian 2002; Farmer and Goldreich 2004; Beresnyak and Lazarian 2008). In this situation, the current-driven instability proposed by Bell (2004) became the central idea being discussed in order to solve the problem of the acceleration of high energy cosmic rays.

Beresnyak, Jones \& Lazarian (2009, henceforth BJL09) suggested an alternative model for enhancing the magnetic field in the preshock region. This model is based on the magnetic field generation within the shock precursor. The
alloweursor originates from cosmic rays that can be returned 


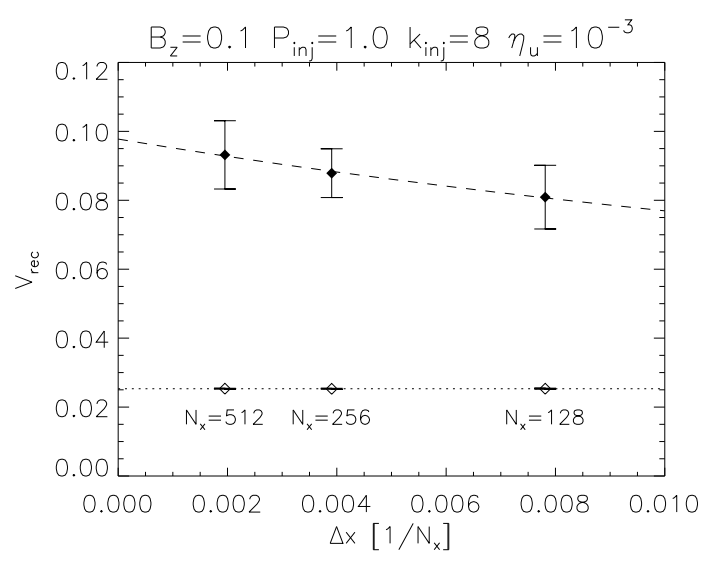

Fig. 16 Dependence of the reconnection rate on the numerical resolution. If the fast reconnection were due to yet unclear numerical effects on small scales, we would expect to see the increase of the reconnection rate with the decrease of the numerical box. If anything, the actual dependence of the reconnection rate on the box size shows the opposite dependence. From Kowal et al. (2012).

back to the shock by the original interstellar magnetic field. The fluid is stirred within the precursor by the combination of fluid density inhomogeneities and CR pressure. This drives vorticity and the precursor turbulence. The latter amplifies magnetic energy through a turbulent dynamo. In other words, BJL09 claims that both the enhancement of magnetic field in the preshock and postshock regions are due to turbulence draining energy from the shock.

The upstream diffusion of CRs leading to precursor formation is associated with a number of possibilities, such as the Drury acoustic instability (Drury 1984; Dorfi and Drury 1985; Kang et al. 1992), which is the enhancement of compressible perturbations by the CR pressure gradient. Such instabilities, however, will only operate on perturbations during the limited time that a fluid element crosses the precursor. This crossing time is rather short $\tau_{c} \approx l_{p} / U_{s}$ where $l_{p}$ is the precursor thickness and $U_{s}$ is the shock velocity and the growth of the density perturbation is constrained.

The difference of the velocities of the fluid elements crossing the shock arises from the difference of the inertia of dense and rarefied gas entering the shock. When an inhomogeneous fluid enters the precursor with speed $u_{0}$ it gets decelerated by the CR pressure gradient until it reaches the speed $u_{1}$ at the dissipative shock front. Let us denote $A_{s}\left(u_{0}-u_{1}\right)$ as the difference between the ballistic velocity of the high-density region and the full decelerated one of the low density regions. The existence of such regions in interstellar medium or solar wind plasma, is expected to arise from the pre-existing turbulence not related to the shock (see 1, Figure 1). The interaction of the precursor with the density inhomogeneities is expected to result in intensive turbulence in the precursor. The turbulence in conducting fluid is known to amplify magnetic fields as depicted in Figure 17

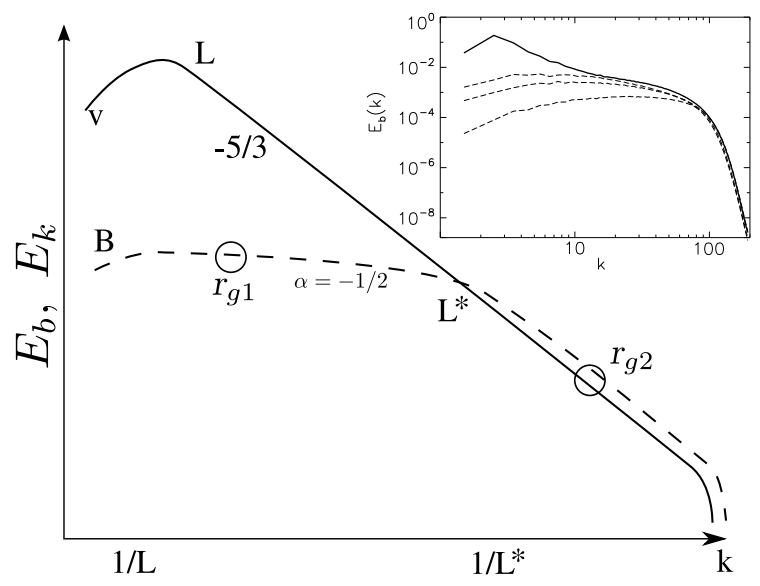

Fig. 17 Magnetic field spectrum (dashed), generated by a small-scale dynamo induced by solenoidal velocity motions (solid). $L^{*}$ is an equipartition scale of magnetic and kinetic motions, it plays a central role in particle scattering. Upper panel: magnetic and velocity fields from simulations (Cho et al. 2009) with different dashed lines corresponding to magnetic spectra at different times. From BJL09.

After extremely short kinematic stage, the energy is growing linearly with time $d E_{B} / d t \approx \alpha_{d} \varepsilon$, where $\varepsilon \sim \rho u_{s}^{3} / L$ is the energy transfer rate, and $\alpha_{d}$ is the efficiency factor $\sim$ 0.06 (Cho et al. 2009). The turbulent dynamo gets magnetic energy in the equipartition with the kinetic energy at a scale $L^{*}$. This scale grows with time. On scales smaller than $L^{*}$ MHD turbulence exists. On scales larger than $L^{*}$, the turbulence is superAlfvénic and velocity has a Kolmogorov spectrum.

As the crossing time of the precursor $\tau_{c}$ is short the generation of magnetic field in the precursors does not get into the saturation regime. The system of equations suggested in BJL09 for the evolutions of magnetic field and $L^{*}$ is as follows:

$\delta B^{2}\left(L^{*}, x_{1}\right)=8 \pi A_{d} \varepsilon \tau\left(x_{1}\right)$

$\frac{\delta B^{*}}{\sqrt{4 \pi \rho}}=u_{s}\left(\frac{L^{*}\left(x_{1}\right)}{L}\right)^{1 / 3}$;

$\tau\left(x_{1}\right)=\int_{x_{1}}^{x_{0}} \frac{d x}{u(x)}$

$L^{*}\left(x_{1}\right)=\left(2 A_{d} u_{s} \tau\left(x_{1}\right)\right)^{3 / 2} L^{-1 / 2}$.

This approach was used to calculate the diffusion coefficients shown in Figure 18

It was shown by Bell (2004) that magnetic fields in the preshock region could be amplified significantly compared to the background field. The model is based on a currentdriven instability. It was successfully tested numerically in a number of papers (Vladimirov et al. 2006; Zirakashvili et al. 2008; Riquelme and Spitkovsky 2009). In Bell (2004) the growth of magnetic field at the linear stage is fastest on the 

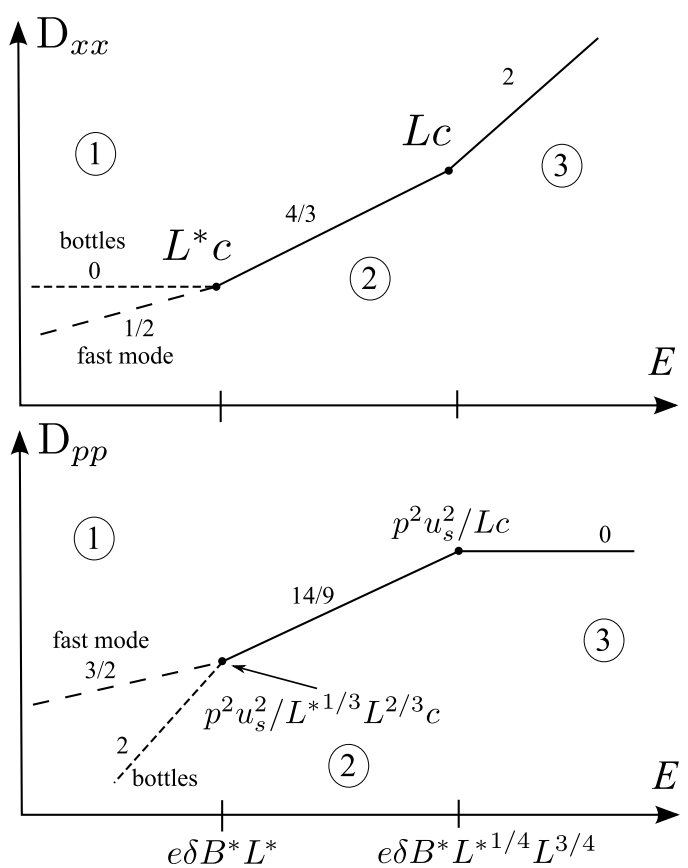

Fig. 18 Diffusion coefficients. (1) is low-energy scattering, which depends on the properties of small-scale MHD turbulence (dashed: fast modes are present; dotted: fast modes are absent); (2) is mediumenergy scattering, by the magnetic fields generated by turbulent dynamo; (3) is high-energy scattering. From BJL09.

characteristic scale that is determined by the initial field $B_{0}$, and the current $j_{d}$,

$l=1 / k_{c}=\frac{c B_{0}}{4 \pi j_{d}}$

where $j_{d}$ arises from high-energy CRs that correspond to $q B_{0} / p c<<k_{c}$. The linear growth rate depends only on the current according to the relation

$\gamma=\frac{j_{d}}{c \sqrt{\frac{\rho_{0}}{\pi}}}$.

BLJ09 demonstrated that, conservatively assuming that the linear growth rate is applicable to the current-driven instability is in the stage with $\delta B \sim B_{0}$, one still gets that the dynamo generation of magnetic fields is dominant, i.e.

$$
\begin{aligned}
\frac{d B_{c u r}^{2}}{d B_{d y n}^{2}} & =1.6 \times 10^{-4}\left(\frac{10^{15} \mathrm{eV}}{E_{\text {esc }}}\right)\left(\frac{\eta_{\text {esc }}}{0.05}\right)\left(\frac{L}{1 \mathrm{pc}}\right) \\
& \times\left(\frac{B_{0}}{5 \mu \mathrm{G}}\right)\left(\frac{v_{A 0}}{12 \mathrm{~km} / \mathrm{s}}\right)\left(\frac{0.5 u_{s h}}{A_{s}\left(u_{0}-u_{1}\right)}\right)^{3} .
\end{aligned}
$$

where $\eta_{\text {esc }} \approx 0.05$ between the flux of CR energy emitted by the shock and the flux of energy of the incoming fluid $\rho u_{s h}^{3} / 2$ (Riquelme and Spitkovsky 2009).

The higher efficiency of the BJL09 model arises from the fact that in this model the full pressure ( $\sim$ energy density) of the CRs is driving the instability, while in the Bell (2004) instability arises only from those CRs that are able to freely stream. The efficiency can be reduced and Bell (2004) instability wins if $A_{s}$ parameter is much less than unity. Further research should clarify the relative importance of the two ways of generating magnetic fields in the precursor. BJL09 estimate that the maximum energy that can be obtained by the acceleration of the shocks with the small-scale dynamo in the precursor is $\sim 10^{16} \mathrm{eV}$, which is consistent with the galactic cosmic ray measurements.

\subsection{Second order Fermi acceleration and turbulence}

To deal with the problem of propagation and acceleration of CRs the so-called diffusive approximation is frequently used. Within this approximation it is assumed that the particle scatter or gain energy in small steps and the dynamics is averaged to obtain the spatial diffusion coefficient, $D_{x x}$, and the momentum diffusion coefficient, $D_{p p}$. The resulting advection-diffusion equation for the evolution of quasiisotropic CR distribution function $f$ is

$$
\begin{aligned}
\frac{\partial f}{\partial t}+u \frac{\partial f}{\partial x} & =\frac{\partial}{\partial x}\left(D_{x x} \frac{\partial f}{\partial x}\right) \\
& +\frac{p}{3} \frac{\partial u}{\partial x} \frac{\partial f}{\partial p}+\frac{1}{p^{2}} \frac{\partial}{\partial p}\left(p^{2} D_{p p} \frac{\partial f}{\partial p}\right),
\end{aligned}
$$

(e.g. Skilling 1975), where for simplicity it is assumed that $f(x, p)$ depends only on one spatial coordinate $x$ and the magnitude of CR momentum, $p$. In Eq. (33) the momentum diffusion corresponds to the second order Fermi acceleration (see Longair 2010).

The diffusion coefficients in Eq. (33) depend on the statistical properties of magnetic turbulence that interacts with the particles. Adopting the decomposition of compressible MHD turbulence in modes (\$2.2) Yan and Lazarian (2002, 2004) identified the fast modes as the principal modes responsible for scattering and turbulent acceleration of CRs in galactic environment. Later analogous conclusions were reached for the CR acceleration in clusters of galaxies (Brunetti and Lazaria 2007).

The above conclusion follows from Alfvén and slow modes being inefficient for resonance interaction with cosmic rays Chandran 2000; Yan and Lazarian 2002). Indeed, as we discussed in 2.2 the two modes are anisotropic with the anisotropy increasing with the decrease of the scale. The resonant interaction of the CRs and Alfvénic perturbations happens when the CR Larmor radius is of the order of the parallel scale of the eddy. As the eddies at scales much less than injection scale are very elongated, the CR samples many uncorrelated eddies, which reduces the interaction efficiency.

Yan and Lazarian (2002) showed that the scattering by fast modes, which are isotropic (CL02), dominates (see Fig. 19]. 

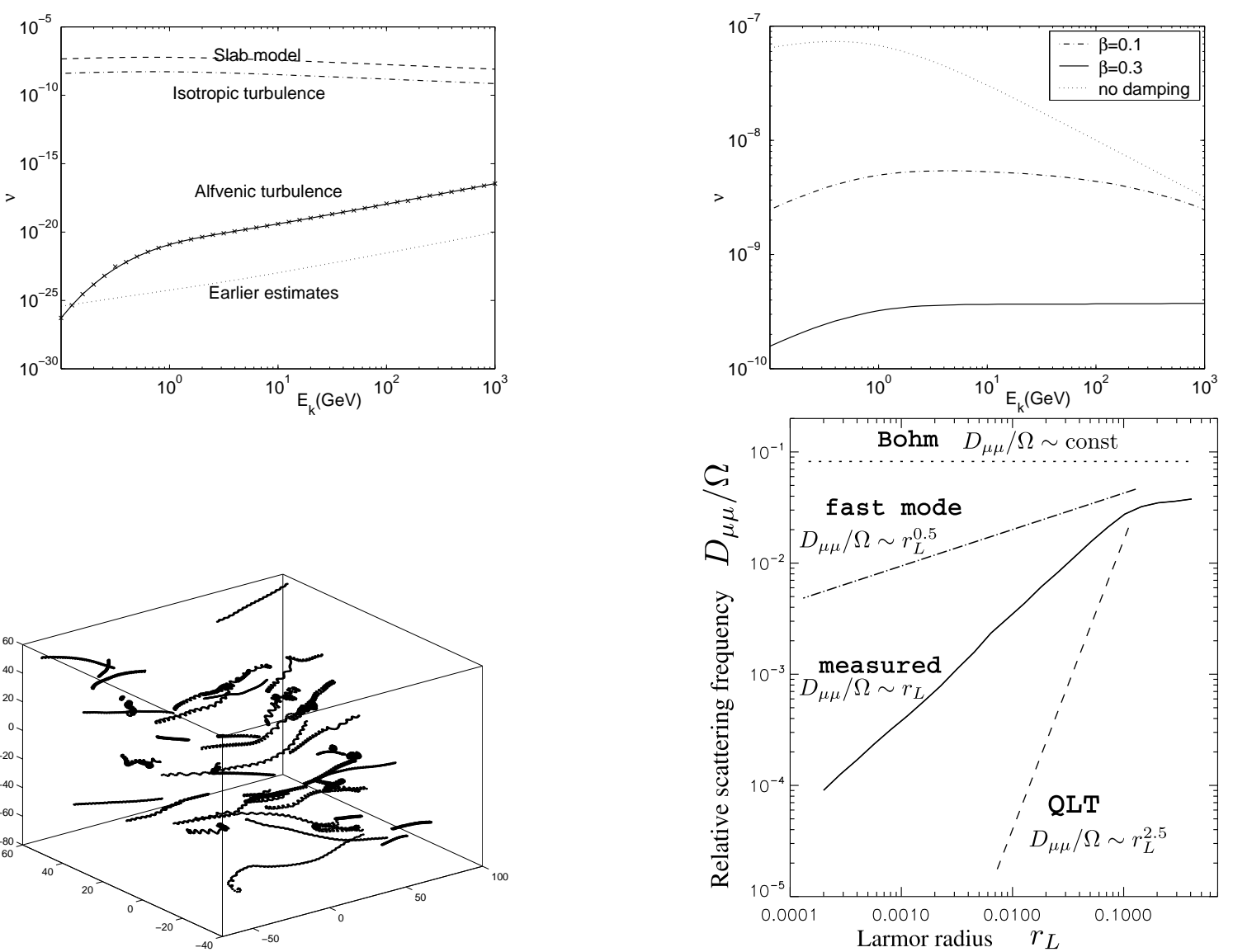

Fig. 19 Implications of turbulence for cosmic rays. Upper left: Rate of CR scattering by Alfvén waves versus CR energy. The lines at the top of the figure are the accepted estimates obtained for Kolmogorov turbulence. The dotted curve is from Chandran (2000). The analytical calculations are given by the solid line with our numerical calculations given by crosses. Upper right: The rate of CR scattering $(v)$ by fast modes in magnetically dominated plasma. The rate of scattering depends on damping of the fast waves (see, which in turn depends on the ratio of gaseous to magnetic pressure $\left(\beta=P_{\text {gas }} / P_{\text {mag }}\right)$. Lower left: Individual trajectories of CRs tracked by the Monte Carlo scattering code. B is obtained through 3-D simulations of MHD turbulence. These calculations provide estimates of CR diffusion. From Yan and Lazarian (2002, 2004). Lower right: Testing of the Yan and Lazarian (2008) non-linear theory with numerical simulations in Beresnyak and Lazarian (2009).

However, fast modes are subject to both collisional and col-

ally, QLT has problems in treating scattering of particles lisionless damping 19 , which was taken into account in Yan and Laxithiamomentum nearly perpendicular to the magnetic field (2004).

More recent studies of cosmic ray propagation and ac(see Jones et al. 1973, 1978; Völk 1973, 1975; Owens 1974; celeration that explicitly appeal to the effect of the fast modes Goldstein 1976; Felice and Kulsrud 2001) and perpendicular transport (see Kóta and Jokipii 2000; Matthaeus et al. include Cassano and Brunetti (2005); Brunetti (2006); Brunetti abo03izarian

(2007); Yan and Lazarian (2008); Yan et al. (2008).

The question of the effect of Alfvén modes is not trivial, however. The calculations that were discussed above use a quasi-linear theory (QLT), whose domain of applicability is limited to very strong fields with small perturbations. The key assumption of QLT is that the particle's orbit is unperturbed, significantly limits its applicability. Addition-

\footnotetext{
19 On the basis of weak turbulence theory, (Chandran 2005) has argued that high-frequency fast waves, which move mostly parallel to magnetic field, generate Alfvén waves also moving mostly parallel to magnetic field. We expect that the scattering by these generated Alfvén modes to be similar to the scattering by the fast modes created by them. Therefore we expect that the simplified approach adopted in Yan and Lazarian (2004) and the papers that followed this one to hold.
}

Various non-linear theories have been proposed to improve the QLT (see Dupree 1966; Völk 1973, 1975; Jones et al. 1973; Goldstein 1976). In the recent paper of Yan and Lazarian (2008, henceforth YL08), a nonlinear theory (NLT) based on the Völk (1975) suggestion was developed. The new formalism was applied to two major processes of CR acceleration in turbulence, namely, to gyroresonance and to the transient time damping (TTD) Fisk et al. (1974); Goldstein et al. (1975).

The efficient particle-wave interactions happen when the condition

$\omega-k_{\|} v \mu=n \Omega \quad n=0, \pm 1,2 \ldots$ 
is satisfied. In Eq. (34) where $\omega$ is the wave frequency, $\Omega=$ $\Omega_{0} / \gamma$ is the relativistic gyration frequency, $\mu=\cos \theta, \theta$ is the pitch angle of particles. TTD corresponds to $n=0$ and it requires compressible perturbations. The most important for the gyroresonance are the interactions with $n=1$.

Contrary to QLT which assumes that the magnitude of the magnetic field stay constant, NLT relaxes this assumption and allows the magnetic field to change in a smooth way. Due to conservation of adiabatic invariant $p_{\perp}^{2} / B$ (see Landau and Lifshitz 1975) the pitch angle will gradually vary, resulting in resonance broadening (Völk 1975). Nonlinear transport (NLT) formalism is based on the replacement of the sharp resonance between waves and particles $\delta\left(k_{\|} v_{\|}-\right.$ $\omega \pm n \Omega$ ) from QLT to the "resonance function" $R_{n}$ (YL08):

$$
\begin{aligned}
R_{n} & =\Re \int_{0}^{\infty} d t e^{i\left(k_{\|} v_{\|}+n \Omega-\omega\right) t-\frac{1}{2} k_{\|}^{2} v_{\perp}^{2} t^{2}\left(\frac{<\delta B_{\|}^{2}>}{B_{0}^{2}}\right)^{\frac{1}{2}}} \\
& =\frac{\sqrt{\pi}}{\left|k_{\|} \Delta v_{\|}\right|} \exp \left[-\frac{\left(k_{\|} v \mu-\omega+n \Omega\right)^{2}}{k_{\|}^{2} \Delta v_{\|}^{2}}\right],
\end{aligned}
$$

The width of the resonance function depends on the strength of the turbulence $\left.\Delta \mu=\Delta v_{\|} / v_{\perp} \simeq \sqrt{\delta B / B}=\sqrt{M_{A}}\right)$. For gyroresonance $(n= \pm 1,2, \ldots)$ the result depends on whether $\mu$ is strongly or weakly perturbed by regular field. If $\mu \gg \Delta \mu$, the result is similar to QLT, because the exponents in Eq. (35) is close to the $\delta$-functions. For $\mu<\Delta \mu$ the result is different. For instance, for the case of $90^{\circ}$ scattering $\mu \rightarrow 0$ and the resonance happens mostly at $k_{\|, \text {res }} \sim \Omega / \Delta v$, while in QLT $k_{\|, \text {res }} \sim \Omega / v_{\|} \rightarrow \infty$.

The change of the efficiency of the gyroresonance scattering and acceleration by Alfvénic modes is not sufficient to affect the conclusions about the inefficiency of the process for low energy cosmic ray scattering by turbulence driven at large injection scales. However, Yan and Lazarian (2008) showed that TTD gets appreciably more efficient, in contrast with the QLT-based result. TTD due to nonlinear scattering can be understood as a scattering by large-scale magnetic compressions arising from the slow mode perturbations.

To test the NLT results particle tracing simulations were performed in Beresnyak et al. (2011). Test particle simulation is the tool extensively used to study CR scattering and transport (e.g. Giacalone and Jokipii 1999; Mace et al. 2000; Oin et al. 2002). The aforementioned studies, however, used synthetic data for turbulent fields which is problematic due to a number of reason. First of all, creating synthetic turbulence data which has scale-dependent anisotropy with respect to the local magnetic field as it is required both by theory and simulations has not been done so far. In addition, synthetic data has used Gaussian statistics and deltacorrelated fields, which is hardly appropriate for description of strong turbulence. Therefore Beresnyak et al. (2011) used the magnetic field obtained as a result of numerical MHD simulations. The results of this testing is shown in Figure 19.
The difference between QLT and NLT has important astrophysical consequences. Indeed, in some phases, such as hot ISM, the fast mode is strongly damped, which makes vital the issue of the interaction of CRs with Alfvén and slow modes. QLT predicts the marginal interaction of these modes with CR. One can view the NLT result for the TTD as the lower limit for the turbulence interaction with CRs.

\subsection{Complexity of turbulent acceleration}

\subsubsection{A network of acceleration sites}

We can view the fragmented acceleration sites as a synthesis of local accelerators (turbulent quasi-perpendicular shocks, Strong turbulence and multi island reconnection) all of them share the same transport characteristics i.e. particles visit sporadically the localized and strong electric fields were the particles "trapped" for a short time (called "stickiness" in non-equilibrium statistical mechanics) and undergo sudden energy jump (called in statistical mechanics "Levy flight"). Vlahos et al. (2004), studied the evolution of particles inside a Network of Acceleration Sites (non-linear structures with random size) associated with (random strength) electric fields. They use three density probabilities mentioned already above to analyze the transport properties of the energetic particles. $P 1\left(\ell_{i}\right)$ represented the density probability for the spacial transport between the "scattering centers or Nodes" of the accelerators.

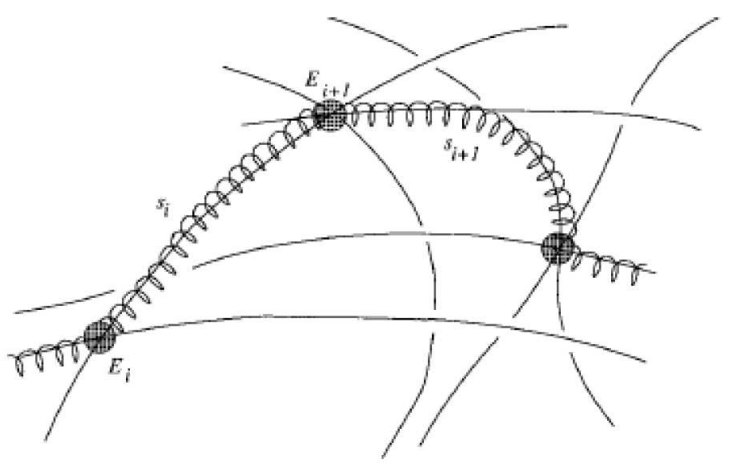

Fig. 20 Sketch of the basic elements of the Network of particle accelerators. A particle (spiraling line) basically follows the magnetic field lines (solid lines), although also undergoing drifts and travel freely a distance $\ell_{i}$ until it enters the "scattering center or acceleration Node" (filled circle), where it is accelerated by the local "electric field $E_{i}$ ". After spending a time $\tau_{i}$ inside the acceleration node it move freely again till it meets the new "Acceleration Node".

The $P(E)$ is the density probability for the energy gain of the particle interacting with the the acceleration sites ("scattering center or the Nodes"), and finally the density probability $P\left(\tau_{i}\right)$ of the time the particle spend inside the "scattering center or the Node". 
Particle acceleration in astrophysics is a multi-scale process. A number of multi-scale systems starting from large scale "turbulent shocks" or "strong turbulence" and go down to the small scale structures (multiple islands and/or current sheets). The fundamental question which we address next is: Is it possible, by following the dynamics of accelerated particle inside the environments discussed above, to reconstruct their transport properties?

\section{Transport properties of the unified accelerator.}

The key problem on the transport properties of particles acceleration is the characteristics of the orbits in a dynamical system close to equilibrium (see Fig. 21 a) and nonequilibrium systems (see Fig. 21b).

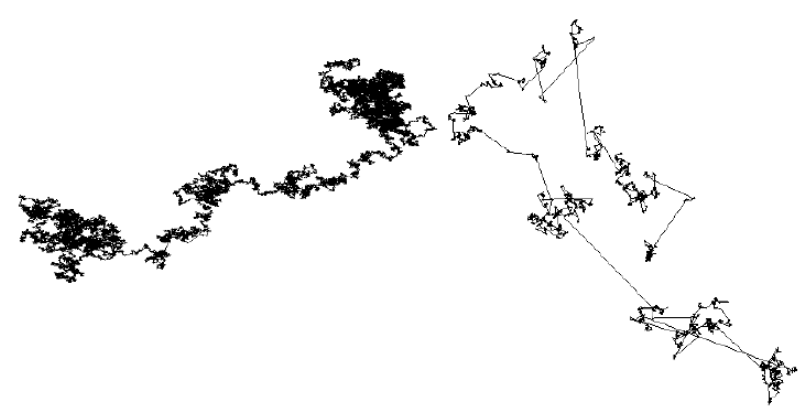

Fig. 21 (a) Random walk in dynamical systems close to equilibrium (normal diffusion:trajectory in the left) (b) Random walk in dynamical systems far from equilibrium (anomalous diffusion: trajectory on the right).

In the one hand we have the well known Brownian motion which is very well studied and analyzed (called in the literature as Normal Diffusion) and in the other hand we see orbits following a mixture of short scale random walks interrupted with large "flights" called in statistical mechanics "Levy flights" and represent in the literature as Anomalous Diffusion (see the tutorial Vlahos et al. (2008) for more details). We present bellow the man characteristics and the dynamics of the orbits and the transport equations for these types of orbits.

Normal diffusion and the Fokker Planck equation.

Deriving the the Fokker Planck equation (or Kolmogorov forward equation) from the characteristics of the density probabilities discussed already is very instructive, that defines its limitations and shows its connection with systems close to equilibrium. We start from a description of diffusion in terms of a random walk, assuming that: (i) the mean value of the random walk steps can be different from zero, which corresponds to a systematic motion of the particles, and (ii) we assume that both the mean and the variance can be spatially dependent, which means that the distribution of increments depends on the spatial location, i.e. it is of the form
$q_{\Delta z, z}(\Delta z, z)$. The probability distribution function for a particle at time $\mathrm{t}$ at the position $\mathrm{z}$ is

$P(z, t)=\int_{\infty}^{\infty} P(z-\Delta z, t-\Delta t) q_{\Delta z, z}(\Delta z, z-\Delta z) d \Delta z$,

which is the Chapman-Kolmogorovequation, and where now $q_{\Delta z, z}(\Delta z, z)$ is the probability density for being at position $z$ and making a step $\Delta z$ in time $\Delta t$. The FP equation can be derived as follows: we expand the integrand of Eq. (36) in a Taylor-series in terms of $z$, so that $P(z, t)=\int_{\infty}^{\infty} A B d \Delta z$, with

$A=P(z, t)-\partial_{t} P(z, t) \Delta t-\partial_{z} P(z, t) \Delta z+\frac{1}{2} \partial_{z}^{2} P(z, t) \Delta z^{2}+\ldots$,

where we have also expanded to first order in $t$,

$B=q_{\Delta z, z}(\Delta z, z)-\partial_{z} q_{\Delta z, z}(\Delta z, z) \Delta z+\frac{1}{2} \partial_{z}^{2} q_{\Delta z, z}(\Delta z, z) \Delta z^{2}+\ldots$

(note that the Taylor expansion is with respect to the second argument of $q_{\Delta z, z}$, we expand only with respect to $z$, not though with respect to $\Delta z$ ). Keeping all terms up to second order in $\Delta z$, we find the FP equation (Vlahos et al. 2008)

$\partial_{t} P(z, t)=-\partial_{z}[V(z) P(z, t)]+\partial_{z}^{2}[D(z) P(z, t)]$,

with $V(z)$ systematic or drift term, and $D(z) \equiv\left\langle\Delta z^{2}\right\rangle(z) / 2 \Delta t$ the diffusion coefficient (Gardiner 1994). The FP equation is also applied to velocity space, e.g. in plasma physics in order to treat collisional effects, or to position and velocity space together. It has the advantage of being a deterministic differential equations that allows to describe the evolution of stochastic systems, as long as the diffusivities and drift velocities are known, and as long as the conditions for its applicability are met.

From its derivation it is clear that the FP equation is suited only for systems close to equilibrium, with just small deviations of some particles from equilibrium, or, in the random walk sense, with just small steps of the particles performing the random walk. A further natural generalization for a diffusion equation in the approach followed here would be not to stop the Taylor expansion in Eqs. (37) and (38) at second order in $z$, but to keep all terms, which would lead to the so-called Kramers-Moyal expansion. More details about the Fokker-Planck equation can be found in the literature Gardiner (1994).

Assuming that $A(z)=0$ and $D(z)=D$ (constant) the FP equation is simple

$\partial_{t} P z, t=\frac{\sigma_{\Delta z}^{2}}{2 \Delta t} \partial_{z}^{2} P(z, t)$

and the solution to it in infinite space is again the Gaussian

$P(z, t)=\frac{1}{\sqrt{4 \pi D t}} e^{-z^{2} / 4 D t}$.

The square mean displacement is

$<z^{2}(t)>=\int z^{2} P(z, t) d z=2 D t$,

which is characteristic for the normal diffusion. 


\section{Anomalous diffusion and the Fractional Diffusion Equa- tion}

Normal diffusion has as basic characteristic the linear scaling of the mean square displacement of the particles with time, $\left\langle r^{2}\right\rangle \sim D t$. Many different experiments though, including the one shown in the previous section, reveal deviations from normal diffusion, in that diffusion is either faster or slower, and which is termed anomalous diffusion. A useful characterization of the diffusion process is again through the scaling of the mean square displacement with time, where though now we are looking for a more general scaling of the form

$\left\langle r^{2}(t)\right\rangle \sim t^{\gamma}$

Diffusion is then classified through the scaling index $\gamma$. The case $\gamma=1$ is normal diffusion, all other cases are termed anomalous. The cases $\gamma>1$ form the family of super-diffusive processes, including the particular case $\gamma=2$, which is called ballistic diffusion, and the cases $\gamma<1$ are the sub-diffusive processes. If the trajectories of a sufficient number of particles inside a system are known, then plotting $\log \left\langle r^{2}>\right.$ vs $\log t$ is an experimental way to determine the type of diffusion occurring in a given system.

As an illustration, let us consider a particle that is moving with constant velocity $v$ and undergoes no collisions and experiences no friction forces. It then obviously holds that $r=v t$, so that $\left\langle r^{2}(t)\right\rangle \sim t^{2}$. Free particles are thus superdiffusive in the terminology used here, which is also the origin of the name ballistic for the case $\gamma=2$. Accelerated particles would even diffuse faster. The difference between normal and a anomalous diffusion is also illustrated in Fig. 21, where in the case of anomalous diffusion long "flights" are followed by efficient "trapping" of particles in localized spatial regions, in contrast to the more homogeneous picture of normal diffusion.

It is to note that anomalous diffusion manifests itself not only in the scaling of Eq. (43) with $\gamma \neq 1$ (which experimentally may also be difficult to be measured), but also in 'strange' and 'anomalous' phenomena such as 'uphill' diffusion, where particles or heat diffuse in the direction of higher concentration, or the appearance of non-Maxwellian distributed particle velocities very often of power-law shape, and it is the main interest in this proposal.

Bian and Browning (2008) developed a simple model for particle acceleration based on the ideas mentioned above and using the Continuous Random Walk developed a more general the fractional diffusion equation (Metzler and Klafter 2000. It is demonstrated clearly (see also the review Vlahos 2008) that depending on the form of the probabilities $\left.P_{(} \ell_{i}\right), P_{2}(E) c B$ n(verging magnetic fluxes, it gains energy through the first we can build a transport equation which tailored on the characteristics of the above probabilities e.g. assuming that $P_{1}, P_{2}, P_{3}$ are Gaussian we recover the FP equation listed above. In the more general case were the probabilities are more gen-

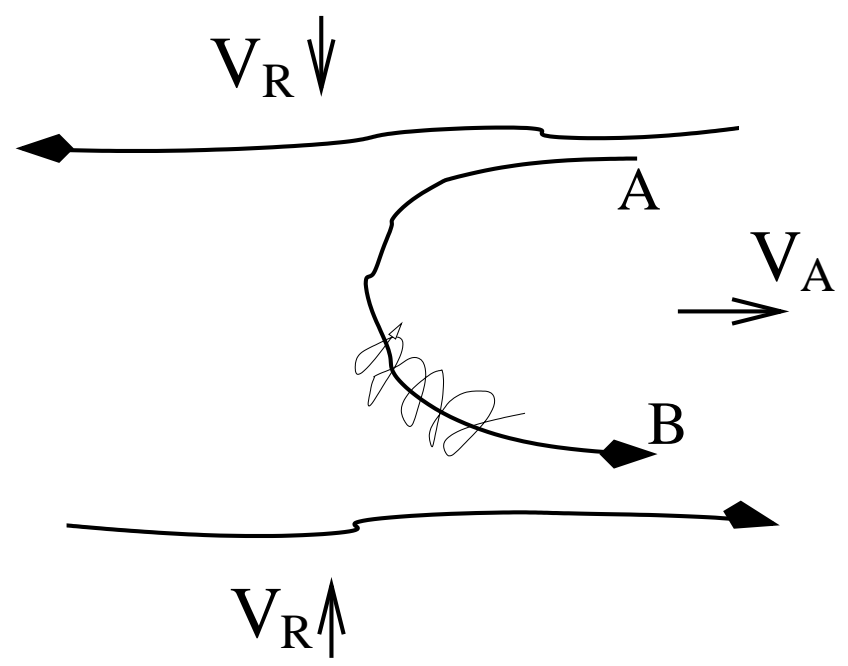

Fig. 22 Cosmic rays spiral about a reconnected magnetic field line and bounce back at points $\mathrm{A}$ and $\mathrm{B}$. The reconnected regions move towards each other with the reconnection velocity $V_{R}$. The advection of cosmic rays entrained on magnetic field lines happens at the outflow velocity, which is in most cases of the order of $V_{A}$. Bouncing at points $A$ and $B$ happens because either of streaming instability induced by energetic particles or magnetic turbulence in the reconnection region. In reality, the outflow region gets filled in by the oppositely moving tubes of reconnected flux which collide only to repeat on a smaller scale the pattern of the larger scale reconnection. From Lazarian (2005).

eral, as we have demonstrated for the case of the quasiperpendicular shock, the strong turbulence and the multiisland reconnection the transport equation for the accelerated particles becomes fractional (Bian and Browning 2008).

Finally, we would like to mention that while in GS95 model of turbulence subdiffusion is difficult to realize Yan and Lazarian (2008), the superdiffusion related to the accelerated divergence of magnetic field lines in space is the expected and confirmed property (LV99, see also Lazarian et al. 2004).

\section{Acceleration of cosmic rays within LV99 model of reconnection}

\subsection{Model of first order Fermi acceleration}

In what follows we discuss the first order Fermi acceleration which arises from volume-filling reconnection 20. The LV99 presented such a model of reconnection and observations of the Solar magnetic field reconnection support the volumefilled idea (Ciaravella and Raymond 2008).

Figure 22 exemplifies the simplest realization of the acceleration within the reconnection region expected within

${ }_{20}$ We would like to stress that Figure 11 exemplifies only the first moment of reconnection when the fluxes are just brought together. As the reconnection develops the volume of thickness $\Delta$ gets filled with the reconnected $3 \mathrm{D}$ flux ropes moving in the opposite directions. 
order Fermi acceleration described in de Gouveia Dal Pino and Lwhicians the spectrum of accelerated energetic particles for (2003, 2005, henceforth GL05) (see also Lazarian 2005).

To derive the energy spectrum of particles one can use the routine way of dealing with the first order Fermi acceleration in shocks (see Longair 1992). Consider the process of acceleration of $M_{0}$ particles with the initial energy $E_{0}$. If a particle gets energy $\beta E_{0}$ after a collision, its energy after $m$ collisions is $\beta^{m} E_{0}$. At the same time if the probability of a particle to remain within the accelerating region is $P$, after $m$ collisions the number of particles gets $P^{m} M_{0}$. Thus $\ln \left(M / M_{0}\right) / \ln \left(E / E_{0}\right)=\ln P / \ln \beta$ and

$$
\frac{M}{M_{0}}=\left(\frac{E}{E_{0}}\right)^{\ln P / \ln \beta}
$$

For the stationary state of accelerated particles the number $M$ is the number of particles having energy equal or larger than $E$, as some of these particles are not lost and are accelerated further. Therefore:

$N(E) d E=$ const $\times E^{-1+(\ln P / \ln \beta)} d E$

To determine $P$ and $\beta$ consider the following process. The particles from the upper reconnection region see the lower reconnection region moving toward them with the velocity $2 V_{R}$ (see Figure 22). If a particle from the upper region enters at an angle $\theta$ into the lower region the expected energy gain of the particle is $\delta E / E=2 V_{R} \cos \theta / c$. For isotropic distribution of particles their probability function is $p(\theta)=$ $2 \sin \theta \cos \theta d \theta$ and therefore the average energy gain per crossing of the reconnection region is

$$
\langle\delta E / E\rangle=\frac{V_{R}}{c} \int_{0}^{\pi / 2} 2 \cos ^{2} \theta \sin \theta d \theta=4 / 3 \frac{V_{R}}{c}
$$

An acceleration cycle is when the particles return back to the upper reconnection region. Being in the lower reconnection region the particles see the upper reconnection region moving with the speed $V_{R}$. As a result, the reconnection cycle provides the energy increase $\langle\delta E / E\rangle_{\text {cycle }}=8 / 3\left(V_{R} / c\right)$ and

$$
\beta=E / E_{0}=1+8 / 3\left(V_{R} / c\right)
$$

Consider the case of $V_{\text {diff }} \ll V_{R}$. The total number of particles crossing the boundaries of the upper and lower fluxes is $2 \times 1 / 4(n c)$, where $n$ is the number density of particles. With our assumption that the particles are advected out of the reconnection region with the magnetized plasma outflow the loss of the energetic particles is $2 \times V_{R} n$. Therefore the fraction of energetic particles lost in a cycle is $V_{R} n /[1 / 4(n c)]=$ $4 V_{R} / c$ and

$$
P=1-4 V_{R} / c \text {. }
$$

Combining Eq. (45), (47), (48) one gets

$N(E) d E=$ const $_{1} E^{-5 / 2} d E$,

the case when the back-reaction is negligible (see GL05, cf. Drury 2012). 21.

The first order acceleration of particles entrained on the contracting magnetic loop can be understood from the Liouville theorem. In the process of the magnetic tubes contraction a regular increase of the particle's energies is expected. The requirement for the process to proceed efficiently is to keep the accelerated particles within the contracting magnetic loop. This introduces limitations on the particle diffusivity perpendicular to the magnetic field direction. The subtlety of the point above is related to the fact that while in the first-order Fermi acceleration in shocks magnetic compression is important, the acceleration via the LV99 reconnection process is applicable even to incompressible fluids. Thus, unlike shocks, it is not the entire volume that shrinks for the acceleration, but only the volume of the magnetic flux tube. Thus high perpendicular diffusion of particles may decouple them from the magnetic field. Indeed, it is easy to see that while the particles within a magnetic flux rope depicted in Figure 22 bounce back and forth between the converging mirrors and get accelerated, if these particles leave the flux rope fast, they may start bouncing between the magnetic fields of different flux ropes which may sometimes decrease their energy. Thus it is important that the particle diffusion both in the parallel and perpendicular directions to the magnetic field stay different. The particle anisotropy which arises from particles preferentially getting acceleration in terms of the parallel momentum may also be important. Similarly, the first order Fermi acceleration can happen in terms of the perpendicular momentum. This is illustrated in Figure 23. There the particle with a large Larmour radius is bouncing back and forth between converging mirrors of reconnecting magnetic field systematically getting an increase of the perpendicular component of its momentum. Both processes take place in reconnection layers.

\subsection{Simulations of the acceleration of cosmic rays by reconnection}

The numerical studies of the cosmic ray acceleration in reconnection regions were performed in Kowal et al. (2011, 2012), where to test the mechanism the data cubes obtained from the models of weakly stochastic magnetic reconnection were used. For a given snapshot we obtain a full configuration of the plasma flow variables (density and velocity) and magnetic field. We inject test particles in such an environment and integrate their trajectories solving the motion equation for relativistic charged particles

$$
\begin{aligned}
& \frac{d}{d t}(\gamma m \mathbf{u})=q(\mathbf{E}+\mathbf{u} \times \mathbf{B}) \\
& 21 \text { The obtained spectral index is similar to the one of Galactic cosmic } \\
& \text { rays. }
\end{aligned}
$$




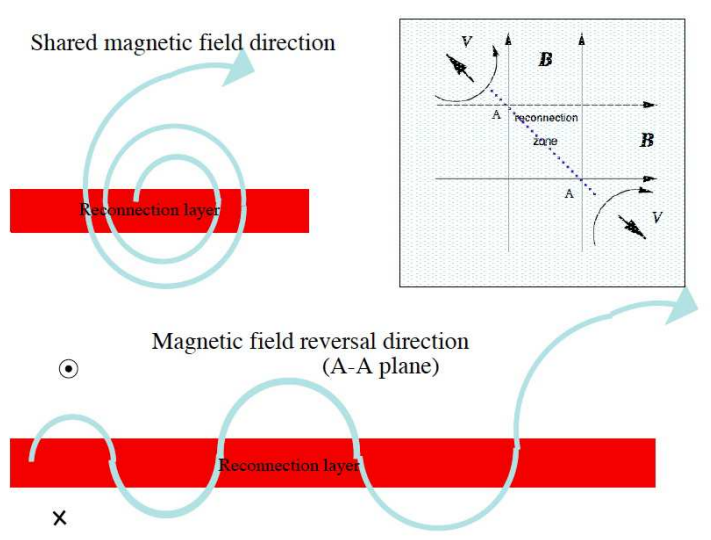

Fig. 23 Particles with a large Larmor radius gyrate about the magnetic field shared by two reconnecting fluxes (the latter is frequently referred to as "guide field". As the particle interacts with converging magnetized flow corresponding to the reconnecting components of magnetic field, the particle gets energy gain during every gyration.

where $\mathbf{u}$ is the particle velocity, $\gamma \equiv\left(1-u^{2} / c^{2}\right)^{-1}$ is the Lorentz factor, $m$ and $q$ are particle mass and electric charge, respectively, and $c$ is the speed of light.

The study of the magnetic reconnection is done using the magnetohydrodynamic fluid approximation, thus we do not specify the electric field $\mathbf{E}$ explicitly. Nevertheless, the electric field is generated either by the flow of magnetized plasma or by the resistivity effect and can be obtained from the Ohm's equation

$\mathbf{E}=-\mathbf{v} \times \mathbf{B}+\eta \mathbf{j}$

where $\mathbf{v}$ is the plasma velocity and $\mathbf{j} \equiv \nabla \times \mathbf{B}$ is the current density.

In our studies we are not interested in the acceleration by the electric field resulting from the resistivity effects, thus we neglect the last term. After incorporating the Ohm's law, the motion equation can be rewritten as

$\frac{d}{d t}(\gamma m \mathbf{u})=q[(\mathbf{u}-\mathbf{v}) \times \mathbf{B}]$

In Figure 24, we present the time evolution of the kinetic energy of the particles which have their parallel and perpendicular (red and blue points, respectively) velocity components accelerated for three models of reconnection. The upper left panel shows the energy evolution for a 2D model without the guide field (as in the models studied in the previous sections). Initially, the particles pre-accelerate by increasing their perpendicular velocity component only. Later we observe an exponential growth of energy mostly due to the acceleration of the parallel component which stops after the energy reaches values of $10^{3}-10^{4} m_{p}$ (where $m_{p}$ is the proton rest mass energy). Further on, particles accelerate their perpendicular component only with smaller linear rate in a log-log diagram. In $2.5 \mathrm{D}$ case, there is also an initial slow acceleration of the perpendicular component fol-
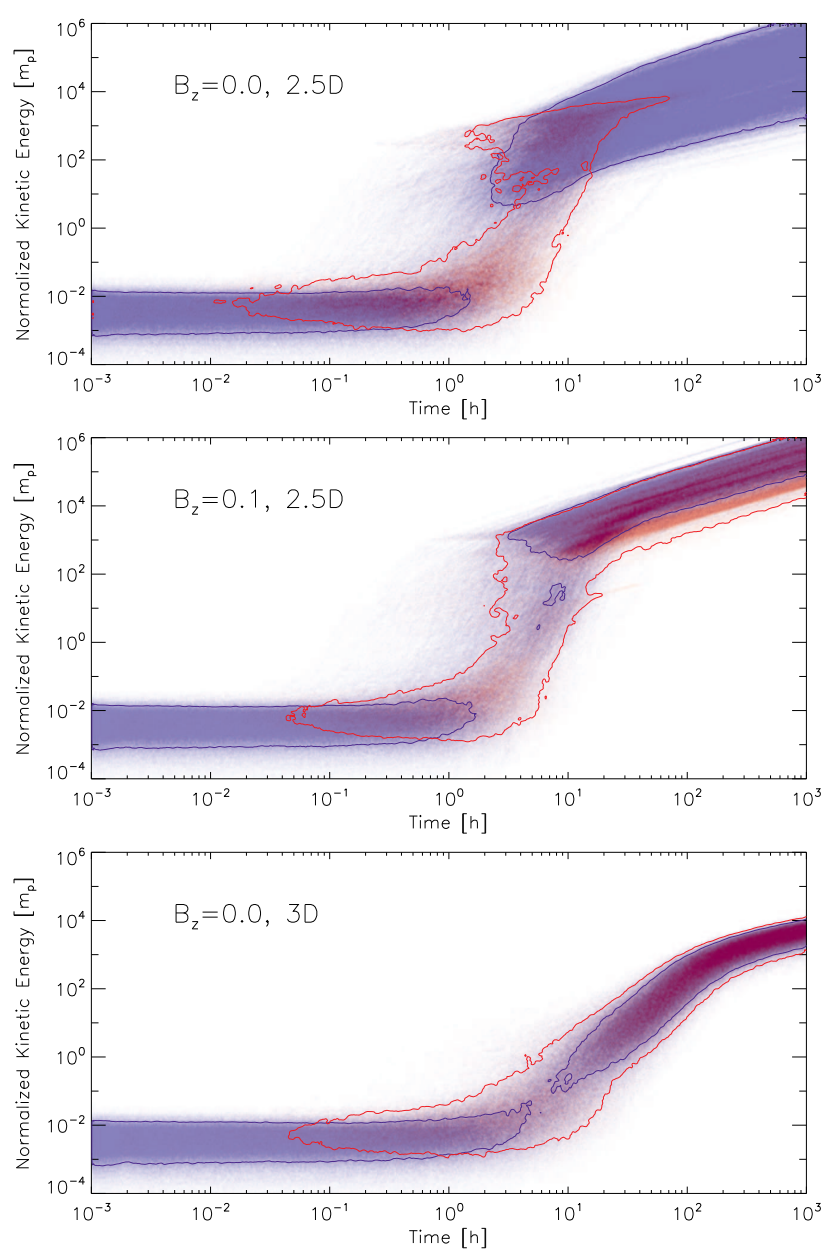

Fig. 24 Kinetic energy evolution of a group of $10^{4}$ protons in 2D models of reconnection with a guide field $B_{z}=0.0$ and 0.1 (top panels, respectively). In the bottom panel a fully $3 \mathrm{D}$ model with initial $B_{z}=0.0$ is presented. The colors show how the parallel (red) and perpendicular (blue) components of the particle velocities increase with time. The contours correspond to values 0.1 and 0.6 of the maximum number of particles for the parallel and perpendicular accelerations, respectively. The energy is normalized by the rest proton mass energy. The background magnetized flow with multiple current sheet layers is at time 4.0 in Alfvén time units in all models. From Kowal et al. (2011).

lowed by the exponential acceleration of the parallel velocity component. Due to the presence of a weak guide field, the parallel component accelerates further to higher energies at a similar rate as the perpendicular one. This implies that the presence of a guide field removes the restriction seen in the 2D model without a guide field and allows the particles to increase their parallel velocity components as they travel along the guide field, in open loops rather than in confined 2D islands. This result is reconfirmed by the 3D model in the bottom panel of Figure 24, where no guide field is necessary as the MHD domain in fully three-dimensional. In this case, we clearly see a continuous increase of both components, which suggests that the particle acceleration behavior 
changes significantly when 3D effects are considered, where open loops replace the closed 2D reconnecting islands.

\subsection{Implications of the acceleration via reconnection}

\subsubsection{Origin of the anomalous cosmic rays}

The processes of the energetic particle acceleration in the process of turbulent reconnection can pre-accelerate particles to the intermediate energies helping to solve the problem of particle injection into shocks. It can also act as the principal process of acceleration. Below we present the case where we believe that the latter takes place.

Since the crossing of the termination shock (TS) by Voyager 1 (V1) in late 2004 and by Voyager 2 (V2) in mid 2007 it became clear that several paradigms needed to be revised. Among them was the acceleration of particles. Prior to the encounter of the termination shock by V1 the prevailing view was that anomalous cosmic rays (ACRs) were accelerated at the TS by diffusive shock acceleration (DSA) to energies 1-300 MeV/nuc (e.g. Jokipii and Giacalone 1998; Cummings and Stone 1998). However, with the crossing of the TS by V1 the energy spectrum of ACR did not unroll to the expected source shape: a power-law at lower energies with a roll off at higher energies. After 2004, both the V1 spectrum in the heliosheath and the V2 spectrum upstream the TS, continued to evolve toward the expected source shape.

To explain this paradox several models were proposed. Among them, McComas and Schwadron (2006) suggested that at a blunt shock the acceleration site for higher energy ACRs would be at the flanks of the TS, where the injection efficiency would be higher for DSA and connection times of the magnetic field lines to the shock would be longer, allowing acceleration to higher energies. Fisk et al. (2006) on the other hand suggested that stochastic acceleration in the turbulent heliosheath would continue to accelerate ACRs and that the high-energy source region would thus be beyond the TS. Other works, such as Jokipii (2006) and Florinski and Zank (2006) try to explain the deficit of ACRs based on a dynamic termination shock. Jokipii (2006) pointed out that a shock in motion on time scales of the acceleration time of the ACRs, days to months, would cause the spectrum to differ from the expected DSA shape. Florinski and Zank (2006) calculated the effect of Magnetic Interacting Regions (MIRs) with the Termination Shock on the ACR spectral shape. They show that there is a prolonged period of depressed intensity in mid-energies from a single MIR. Other recent works have included stochastic acceleration, as well as other effects (Moraal et al. 2006; Zhang 2006; Langner and Potgieter 2006; Ferreira et al. 2007). It became clear after the crossing of the TS by V2 that these models would require adjustments. The observations by V2 indicate for example that a transient did not cause the modulation shape of the V2 spectrum at the time of its TS crossing. When both spacecraft were in the heliosheath in late 2007, the radial gradient in the 13-19 MeV/nuc ions did not appear to be caused by a transient. The 60-74 MeV/nuc ions have no gradient, so no north-south or longitudinal asymmetry is observed in the ACR intensities at the higher energies.

In Lazarian and Opher (2009, henceforth LO09) we propose an alternative model, which explains the source of ACRs as being in the heliosheath and we appeal to magnetic reconnection as a process that can accelerate particles. LO09 explained the origin of the magnetic field reversals that induce magnetic reconnection in heliosheath and heliopause.

Indeed, it is well known that magnetic field in the heliosphere change polarity and induce reconnection. For instance, as the Sun rotates magnetic field twists into a Parker spiral (Parker 1958) with magnetic fields separated by a current sheet (see Schatten 1971). The changes of magnetic field are also expected due to the Solar cycle activity.

The question now is at what part of the heliosheath we expect to see reversals. The structure of the magnetic field in the solar wind is complex. The solar magnetic field lines near the termination shock are azimuthal and form a spiral (see Figure 25). We expect the reconnection and the corresponding energetic particle acceleration to happen at the heliosheath closer to the heliopause. This explains why Voyagers do not see the signatures of anomalous cosmic ray acceleration as they pass the termination shock. Appealing to their model of collisionless reconnection, Drake et al. (2010) provided a similar explanation of the origin of the anomalous cosmic rays.

\subsubsection{Acceleration of cosmic rays in heliototail}

It is known that cosmic rays arrival direction has an energy dependent large angular scale anisotropy with an amplitude of order $10^{-4}-10^{-3}$. The first comprehensive observation of this anisotropy was provided by a network of muon telescopes sensitive to sub-TeV energies and located at different latitudes (Nagashima et al. 1998). More recently, an anisotropy was also observed in the multi-TeV energy range by the Tibet AS $\gamma$ array (Amenomori et al.2006), Super-Kamiokande (Guillian et al. 2007) and by MILAGRO (Abdo et al. 2009), and the first high statistics observation in the southern hemisphere in the $10 \mathrm{TeV}$ region, is being reported by IceCube (Abbasi et al. 2010). The origin of the large angular scale anisotropy in the cosmic rays arrival direction is still unknown. The structure of the local interstellar magnetic field is likely to have an important role. However the combined study of the anisotropy energy and angular dependency, its time modulation and angular scale structure seem to suggest that the observation might be a combination of multiple superimposed effects, caused by phenomenologies at different distances from Earth. 


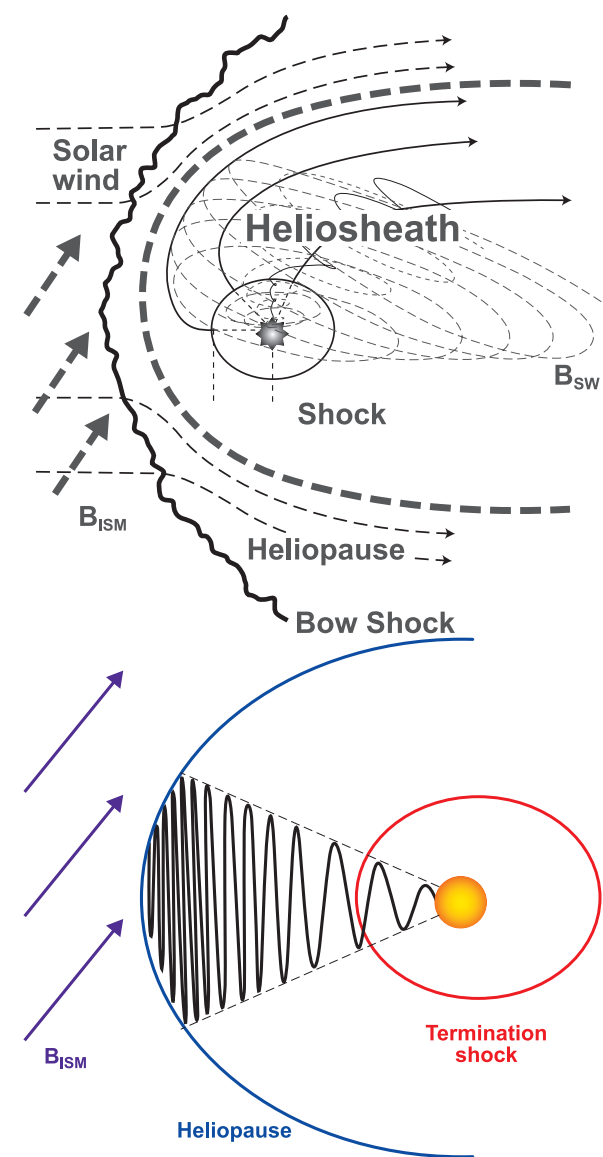

Fig. 25 Upper plot. Global view of the interaction of the solar wind with the interstellar wind. The spiral solar magnetic field (shown in dark dashed lines) is shown being deflected at the heliopause. The heliopause itself is being deflected by the interstellar magnetic field. (figure adapted from Suess and Nernev 2006). Lower plot. A meridional view of the boundary sectors of the heliospheric current sheet and how the opposite sectors get tighter closer to the heliopause. The thickness of the outflow regions in the reconnection region depends on the level of turbulence. From LO09.

In this context, particular interest is derived from the observation of a broad excess of sub-TeV cosmic rays in a portion of the sky compatible with the direction of the heliospheric tail (or heliotail) (Nagashima et al. 1998; Hall et al. 1999). The heliotail is the region of the heliosphere downstream the interstellar matter wind delimited within the heliopause, i.e. the boundary that separates the solar wind and interstellar plasmas (Izmodenov and Kallenbach 2006). The observed excess was attributed to some unknown anisotropic process connected with the heliotail (thus called tail-in excess). The gyro-radius of sub-TeV cosmic protons is less than about $200 \mathrm{AU}$ (in a $\sim 1 \mu \mathrm{G}$ interstellar magnetic field), which is approximately the size of the heliosphere and, most likely, smaller than the width and length of the heliotail. The persistence of the cosmic ray anisotropy structure in the multi-TeV energy range makes it challenging to link this observation to the heliosphere. Although the unknown size and

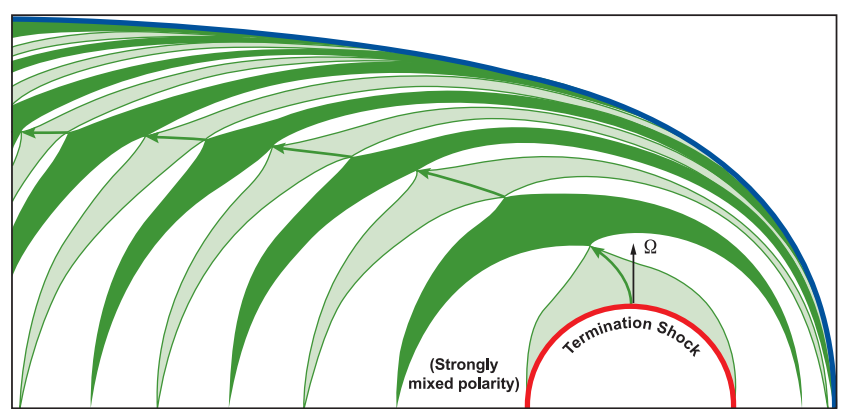

Fig. 26 A meridional view of the boundary sectors of the heliospheric current sheet and how the opposite sectors get tighter closer to the heliopause and into the heliotail. The thickness of the outflow regions in the reconnection region depends on the level of turbulence. The length of the outflow regions $L$ depends on the mean geometry of magnetic field and turbulence. From Lazarian and Desiati (2010).

extension of the heliotail contributes to the uncertainty on the energy scale at which heliospheric influence on cosmic rays starts to be negligible. However, we know that the observations of multi-TeV cosmic rays anisotropy show small angular scale patterns superimposed to the smooth broad structure of the tail-in excess, which is suggestive of a local origin, i.e. within the heliotail. With the same technique used in gamma ray detection to estimate the background and search for sources of gamma rays, the MILAGRO collaboration discovered two localized excess regions in the cosmic rays arrival direction distribution (Abdo et al. 2008). The same excess regions were reported by the ARGO-YBJ air shower array (Vernetto et al. 2009). The strongest and more localized of them (with an angular size of about $10^{\circ}$ ) coincides with the direction of the heliotail.

The explanation of this excess related to the acceleration of energetic particles in reconnection region was suggested by Lazarian and Desiati (2010). Figure 26 represents the possible structure of the heliotail which arises from the solar magnetic field cycles (Parker 1979). The magnetic fields of the opposite polarities emerge as the result of 11 year solar dynamo cycle. As the magnetic field is carried away by solar wind, the reversed magnetic field regions get accumulated in the heliotail region. This is where reconnection is expected to occur.

Naturally, the actual heliotail is going to be turbulent, which is not represented by the idealized drawing in Figure 26. As the Alfvén speed is smaller than the Solar wind speed, magnetic reconnection does not change the overall magnetic field structure. Nevertheless, the effects of turbulence are expected to be very important from the point of view of magnetic reconnection and the particle acceleration that it entails.

The simulations of the heliotail are extremely challenging (see Pogorelov et al. 2009a 2009b) and have not been done with the sufficient resolution and extent. While we believe that future research will provide details necessary for 
quantitative modeling, the schematic representation of the heliotail structure depicted in Figure 26 is true in terms of major features.

Galactic cosmic rays entering the heliotail will get reaccelerated, which would affect their spectrum. The estimate of the maximal energy of protons which can be accelerated through this process can be obtained through the usual arguments that the Larmor radius should not be larger than the size of the magnetized region $L_{z o n e}$ (Longair 1997)

$E_{\text {max }} \approx 0.5 \mathrm{TeV}\left(\frac{B}{1 \mu \mathrm{G}}\right)\left(\frac{L_{\text {zone }}}{100 \mathrm{AU}}\right)$,

which sets the limit of energies, i.e. $E_{\max }$, through appealing to the fact that protons of larger energies cannot be confined by magnetic fields to experience the acceleration through multiple bouncing back and forth between the reconnecting magnetic fluxes. The magnetic field estimates vary and can be expected of the order of $\sim 4 \mu \mathrm{G}$. $L_{z o n e}$ is in the range from 100 to 400 AU (Pogorelov et al. 2009a). Therefore cosmic rays with energies up to $10 \mathrm{TeV}$ can be re-accelerated in the heliotail, explaining the observation 22 .

\subsubsection{Acceleration of particles by reconnection in solar flares and galaxy clusters}

Acceleration is known to accompany solar flares. LV99 explains solar flares and predicts that only insubstantial portion of energy goes into heating during the reconnection. The rest goes into turbulence and the acceleration of energetic particles. The resulting turbulence can accelerate the energetic particles (Petrosian et al. 2008) via the second order Fermi process (see $₫ 6.2$ ), but the first order Fermi acceleration arising from reconnection (see 87.1 ) is also present and may dominate. The relative role of the two processes requires more studies.

LV99 reconnection was discussed as the source of the observed energetic particles in Lazarian (2006) and Lazarian and (2011). Magnetic fields of different polarity get into contact as magnetized gas is being collected into the potential well of the galaxy cluster. In addition, superAlfvénic turbulence in galaxy clusters would produce magnetic field reversals of magnetic fields which will also dissipate through reconnection. Therefore we believe that the acceleration may be efficient. A quantitative study of the process is presented in Brunetti \& Lazarian (2012, in prep.).

\subsubsection{Acceleration in AGNs}

Cosmic ray acceleration in the high energy range is still not fully understood. The spectrum of the highest energy cosmic rays (UHECRs) is consistent with an origin in extragalactic

\footnotetext{
22 An alternative explanation based on scattering cosmic rays in the heliotail was suggested in Desiati and Lazarian (2011)
}

astrophysical sources and candidates range from the birth of compact objects to explosions related to gamma-ray bursts (GRBs), or to events in active galaxies (AGNs) (e.g. Melrose 2009; Kotera and Olinto 2011). Very high energy observations of AGNs and GRBs with the Fermi and Swift satellites and ground based gamma ray observatories (HESS, VERITAS and MAGIC) on the other hand, are challenging current theories of particle acceleration - mostly based on stochastic acceleration in shocks - which have to explain how particles are accelerated to $>\mathrm{TeV}$ energies in regions relatively small compared to the fiducial scale of their sources (Sol et al. 2012, in prep.).

Traditionally discussed predominantly in the context of solar flares (e.g. Drake et al. 2006, 2009; Gordovskyy et al. 2010; Nishizuka and Shibata 2010; Gordovskyy and Browning 2011; Zharkova et al. 2011), the Earth magnetotail (Lazarian and Opher 2009; Drake et al. 2010), and the solar wind (e.g. Lazarian and Desiati 2010), particle acceleration in magnetic reconnection sites is currently also being explored in relativistic astrophysical environments. It has been invoked in the production of ultra high energy cosmic rays (e.g. de Gouveia Dal Pino and Lazarian 2000, 2001; Kotera and Olinto 2011), in jet-accretion disk systems (de Gouveia Dal Pino et al. 2010a 2010b; Giannios 2010; del Valle et al. 2011; Ding et al. 2010), and in the general framework of AGNs and GRBs (Ostrowski 2002; Lazarian et al. 2003; de Gouveia Dal Pino et al. 2010a; Giannios 2010; Zhang and Yan 2011; Uzdensky 2011; Uzdensky and McKinney 2011; de Gouveia Dal Pin 2011; McKinney and Uzdensky 2012).

Magnetic reconnection events like those associated to solar flares can be a very powerful mechanism operating on accretion disks (G05). In fact, the magnetic power released in fast reconnection flares has been found to be more than sufficient to accelerate relativistic plasmons and produce the observed radio luminosity of the nuclear jets associated both to microquasars and low luminous AGNs. The observed correlation between the radio luminosity and the black hole ndibassomitihese sources, which spans $10^{9}$ orders of magnitude in mass (Falcke et al. 2004), is naturally explained in this model as simply due to the magnetic reconnection activity at the jet launching region of the accretion disk coronae of these sources (de Gouveia Dal Pino et al. 2010a 2010b). A similar process may also explain the observed $x$-ray flares in YSOs.

\subsection{Acceleration and Gamma Ray Bursts}

In the field of Gamma Ray Bursts (GRBs), the new observations that followed the launching of Swift and Fermi satellites, while solving some old problems, have raised new questions. The most important unknown parameter is the ratio $(\sigma)$ between the Poynting flux and the matter (baryonic + leptonic) flux. In the standard fireball internal shock (IS) scenario (Paczynski 1986; Shemi and Piran 1990), magnetic 
fields are assumed not to play a dynamically important role, i.e. $\sigma<<1$. An alternative view is that the GRB outflow carries a dynamically important magnetic field component, i.e. $\sigma>>1$. The GRB radiation in this case would be powered by dissipation of the magnetic field energy in the ejecta (e.g. Usov 1992; Thompson 1994; Meszaros and Rees 1997; Piran 1999, 2005; Lyutikov and Blandford 2003). Several recent developments suggest that we should consider seriously magnetic dominated jets as a viable option for the source of GRBs. First, the analogy with AGNs jets in which it is quite certain that the inner engine cannot accelerate baryonic dominated jets suggest that similarly in GRBs at least in its inner region the jet is Poynting flux dominated.

Recent Fermi observation of GRB 080916C shows that the bright photosphere emission associated with a putative fireball is missing, which raises a challenge to the traditional fireball IS model and suggests that the central engine likely launches a Poynting-flux-dominated outflow at least for this burst.

In this case, magnetic energy may be sufficient to feed GRBs. Magnetic reconnection was suggested as a component for GRBs more than a decade ago (Thompson 1994). The problem lay, however, in the intrinsic difficulty of reconnection as it is a very slow process in ordered fields. As with the case for solar flares, both a slow phase of accumulation of the oppositely directed flux and a fast bursty phase are required for reconnection. Essential progress was made by (Lazarian et al. 2003), who proposed a new GRB model of self-adjusted reconnection based on the findings of fast reconnection in 3D turbulent magnetic fields (Lazarian and Vish 1999). As a result of the increased turbulence the reconnection rate increases, inducing a positive feedback which results in the explosive reconnection. Such a process provides an alternative explanation to gamma ray bursts (Lazarian et al. 2003). More recently the model was elaborated and connected with observational data in Zhang and Yan (2011).

Similar to the internal shock model, the mini-shells interact internally at the radius $R_{\mathrm{IS}} \sim \Gamma^{2} c \Delta t$. Most of these early collisions, however, have little energy dissipation, but serve to distort the ordered magnetic field lines entrained in the ejecta. As in other astrophysical objects, the system is prone to turbulence because of high Reynolds and magnetic Reynolds number 23 Since in GRBs, the outflow is highly relativistic, it is impractical for the magnetic field to be distorted. In any case, fast reconnection can be triggered in weakly stochastic magnetic field (LV99). The details require further studies especially in the regime of relativistic turbulence.

\footnotetext{
${ }^{23}$ This is a nontrivial statement given the fact that the system is highly collisionless. Taking into account, nevertheless, the high magnetization factor, most kinetic motions are concentrated perpendicular to magnetic field so that it is the much suppressed perpendicular viscosity and resistivity that should be adopted.
}

The perturbations build up as the mini-shells propagate outward (see Fig 27 right). At a certain point, the turbulence reaches the critical for a fast reconnection to take place. Reconnection events rapidly eject outflow, which further increase the turbulence intensity. This results in a run-away discharge of the magnetic field energy in a reconnection/turbulence avalanche. This is one ICMART event, which corresponds to one GRB pulse. During the magnetic field energy discharge, the $\sigma$ value drops from the original value to around unity.

A GRB is composed of several ICMART events (i.e. broad pulses). The peak energy $E_{p}$ is expected to drop from high to low across each pulse. The $\gamma$-ray polarization degree is also expected to drop from $\sim 50-60 \%$ to $\sim$ a few $\%$ during each pulse. The magnetic field configuration at the end of prompt emission is largely randomized, but still has an ordered component. The GRB light curves should have two variability components, a broad (slow) component related to the central engine activity, and a narrow (fast) component associated with the relativistic magnetic turbulence (see Fig 27). More detailed discussions can be found in Zhang and Yan (2011).

\section{Discussion}

\subsection{Turbulence and cosmic ray acceleration in astrophysical fluid}

Turbulence is the essential part of astrophysical fluid dynamics As astrophysical turbulence is magnetized, MHD turbunniagce presents the most important process to be accounted for. The progress in understanding MHD turbulence calls for the adequate representation of the turbulence in the process of cosmic ray propagation and acceleration. It is regretful that a number of outdated ad hoc ideas about MHD turbulence are still being used while describing cosmic ray interactions with turbulence.

The decomposition of MHD turbulence into modes (Alfvén, fast and slow) provides a way to describe the cosmic ray turbulence interactions in a theory-motivated way. For instance, for the cascade driven at large scales the Alfvén modes get marginally important for scattering of low energy cosmic rays. This is contrary to the claims in most of the textbooks where Alfvén modes are described as the principal scattering agent. On the contrary, the scattering by fast modes is efficient, but the damping of fast modes must be taken into account. This changes the treatment of acceleration compared to the accepted one. At the same time, the ranges of sonic and Alfvén Mach number for which the decomposition works require further studies.

Turbulence is also important for the acceleration of cosmic rays in shocks. The generation of magnetic fields both in the pre-shock and post-shock plasmas are important ingredients of forming strong enough converging mirrors between 


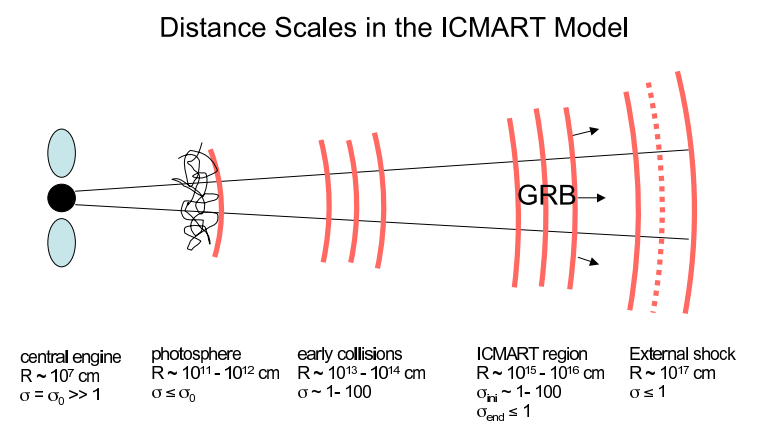

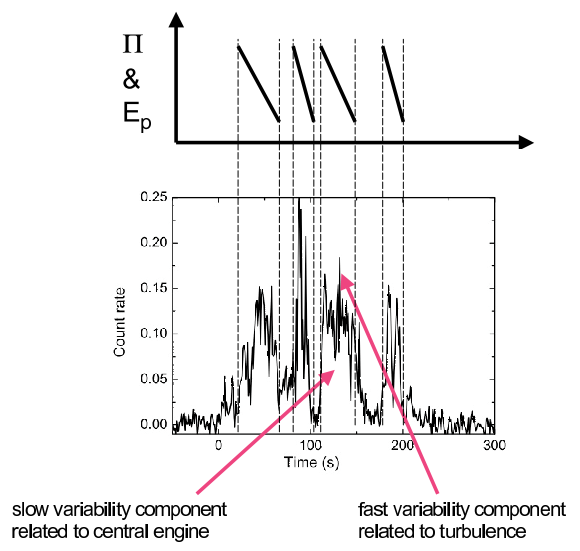

Fig. 27 Left panel: A cartoon picture of the ICMART model. The typical distances and $\sigma$ values of various events are marked. Right panel: An example of GRB light curve that shows two variability time scales. The light curve of GRB 050607 is taken from the NASA Swift GRB archival data web site http://swift.gsfc.nasa.gov/docs/swift/archive/grb_table/ grb_lookup.php?grb_name=050607. The predictions of decreasing gammaray polarization degree $\Pi$ and the spectral peak energy $E_{p}$ within individual pulses are indicatively presented. Detailed decaying functions would be different depending on the details of evolution of magnetic field configuration, $\sigma$ value, as well as balance between heating and cooling of electrons. The general decreasing trend is robust. (from Zhang and Yan 2011)

which cosmic rays bounce and get accelerated. In addition, turbulence makes reconnection of magnetic fluxes fast and this induces the acceleration of cosmic rays via through yet another process of the First order Fermi acceleration type.

A sober note is also due. One has to accept that magnetic turbulence is far from being completely understood phenomenon. There are different regimes of turbulence, e.g. turbulence in partially ionized gas presents a pronouncedly different behavior compared to the standard MHD turbulence picture. Similarly, turbulence in the presence of localized sources and sinks of turbulent energy, i.e. imbalanced turbulence, is rather different from the balanced counterpart. In addition, the instabilities of cosmic ray fluid and the backreaction of cosmic rays on turbulence may also be important. The consequences of these changes on the first order Fermi acceleration and the second order Fermi acceleration require further studies.

\subsection{Models of reconnection}

Since the introduction of the LV99 model, more traditional approaches to reconnection have been changed considerably. At the time of its introduction, the models competing with LV99 were some modifications of a single X-point collisionless reconnection. Those models had point-wise localized reconnection region and inevitably prescribed opening of the reconnection region upon the scales comparable to $L$ (see Figure 11). Such reconnection was difficult to realize in astrophysical conditions in the presence of random forcing which at high probability would collapse the opening of the reconnection layer. Single X-point reconnection were re- jected in observations of solar flares by Ciaravella and Raymond (2008).

Modern models of collisionless reconnection resemble the original LV99 model in a number of respects. For instance, they discuss, similarly to the LV99, the volume filled reconnection, although one may still wonder how this volume filling is being achieved in the presence of a single reconnection layer (see Drake et al. 2006). While the authors still talk about islands produced in the reconnection, in three dimensions these islands are expected to evolve into contracting 3D loops or ropes (Daughton et al. 2008), which is similar to what is depicted in Figure 22. Thus we do not expect to see a cardinal difference between the first order Fermi processes of the acceleration described in GL03 and later in Drake et al. (2006). This suggests that the back-reaction of the particles calculated in Drake et al. (2006) considering the firehose instability may be employed as a part of the acceleration process described in GL03.

The departure from the idea of regular reconnection and introduction of magnetic stochasticity is also obvious in a number of the recent papers appealing to the tearing mode instability 24 as the process of enhancing reconnection (Loureiro et al. 2009; Bhattacharjee et al. 2009). The 3D loops that should arise as a result of this process should be able to accelerate

${ }^{24}$ The idea of appealing to the tearing mode as a means of enhancing the reconnection speed can be traced back to Strauss (1988), Waelbroeck (1989) and Shibata and Tanuma (2001). LV99 showed that the linear growth of tearing modes is insufficient to obtain fast reconnection. The new attack on the problem assumes that the non-linear growth of the islands due to merging provides their growth rates at the large scales that are larger than the direct growth of the tearing modes at those scales. This situation when the non-linear growth is faster than the linear one is rather unusual and requires further investigation. 
energetic particles via the process described in GL03. As tearing modes can happen in a collisional fluid, this may potentially open another channel of reconnection in such fluid. The limitation of this process is that the tearing mode reconnection should not be too fast as this would present problems with explaining the accumulation of the flux prior to the flare. At the same time the idea of tearing reconnection does not have the natural valve of enhancing the reconnection speed, which is contrary to the LV model where the degree of reconnection is determined by the level of turbulence. Thus the periods of slow reconnection in LV99 model are ensured by the low level of turbulence prior to the flare. We believe that tearing reconnection can act to destabilize the laminar Sweet-Parker reconnection layer, inducing turbulence. In this sense the new ideas on tearing instability may be complementary to the LV99 model.

We note, however, that in most astrophysical situations one has to deal with the pre-existing turbulence, which is the consequence of high Reynolds number of the fluid. Such turbulence may modify or suppress instabilities, including the tearing mode instability. We claim that it, by itself, induces fast reconnection. We may claim that even if the astrophysical fluid is kept initially laminar the fluid a thick outflow from the reconnection region caused by tearing is expected to become turbulent. It was argued in Lapenta and Lazarian (2012) that this may be the cause of the reconnection explosions reported recently within MHD simulations (Lapenta 2008; Bettarini and Lapenta 2010).

\section{Summary}

The results of this review can be briefly summarized as follows:

- Astrophysical turbulence is ubiquitous and it plays the essential role for both acceleration of cosmic rays and magnetic reconnection.

- Advances in understanding of MHD turbulence help understanding both cosmic ray acceleration and reconnection.

Our understanding of MHD turbulence in its different regimes is still incomplete, however.

- Turbulence makes magnetic reconnection fast. The LV99 model that describes the process has been successfully tested numerically.

- Preexisting in the media density fluctuations generate magnetic field while interacting with the shock precursor. This increases the efficiency of cosmic ray acceleration and in some instances can even bootstrap the process of acceleration.

- The turbulent second order Fermi acceleration is important for many environments. The standard Quasi Linear Theory (QLT) is not accurate to describe the process, but it can be improved; the improved theory corresponds to numerical simulations.
- The LV99 model of reconnection entails the first order Fermi acceleration of energetic particles. Numerical simulations support this conclusion.

- A number of processes, e.g. the acceleration of anomalous cosmic rays within the magnetosphere, acceleration of cosmic rays in magnetotail as well as in galaxy clusters can be attributed to the acceleration caused by magnetic reconnection.

Acknowledgements A. Lazarian's research is supported by the NASA Grant NNX09AH78G, as well as the support of the NSF Center for Magnetic Self-Organization. The Humboldt Award at the Universities of Cologne and Bochum, as well as Vilas Associate Award and the hospitality of the International Institute of Physics (Brazil) are acknowledged. G. Kowal's research is supported by FAPESP (Brazil) grant no. 2009/50053-8. E. de Gouveia Dal Pino also acknowledges partial supported from FAPESP (Brazil: grant no. 2006/50654-3) and from CNPq (Brazil; grant no. 300083/94-7). We thank the anonymous referee for comments and suggestions that improved the paper.

\section{References}

R. Abbasi, Y. Abdou, T. Abu-Zayyad, J. Adams, J.A. Aguilar, M. Ahlers, K. Andeen, J. Auffenberg, X. Bai, M. Baker, et al., Measurement of the Anisotropy of Cosmic-ray Arrival Directions with IceCube. Astrophys. J. Lett. 718, 194-198 (2010). doi: $10.1088 / 2041-8205$

A.A. Abdo, B. Allen, T. Aune, D. Berley, E. Blaufuss, S. Casanova, C. Chen, B.L. Dingus, R.W. Ellsworth, L. Fleysher, R. Fleysher, M.M. Gonzalez, J.A. Goodman, C.M. Hoffman, P.H. Hüntemeyer, B.E. Kolterman, C.P. Lansdell, J.T. Linnemann, J.E. McEnery, A.I. Mincer, P. Nemethy, D. Noyes, J. Pretz, J.M. Ryan, P.M.S. Parkinson, A. Shoup, G. Sinnis, A.J. Smith, G.W. Sullivan, V. Vasileiou, G.P. Walker, D.A. Williams, G.B. Yodh, Discovery of Localized Regions of Excess 10-TeV Cosmic Rays. Physical Review Letters 101(22), 221101 (2008). doi:10.1103/PhysRevLett.101.221101

A.A. Abdo, B.T. Allen, T. Aune, D. Berley, S. Casanova, C. Chen, B.L. Dingus, R.W. Ellsworth, L. Fleysher, R. Fleysher, M.M. Gonzalez, J.A. Goodman, C.M. Hoffman, B. Hopper, P.H. Hüntemeyer, B.E. Kolterman, C.P. Lansdell, J.T. Linnemann, J.E. McEnery, A.I. Mincer, P. Nemethy, D. Noyes, J. Pretz, J.M. Ryan, P.M.S. Parkinson, A. Shoup, G. Sinnis, A.J. Smith, G.W. Sullivan, V. Vasileiou, G.P. Walker, D.A. Williams, G.B. Yodh, The LargeScale Cosmic-Ray Anisotropy as Observed with Milagro. Astrophys. J. 698, 2121-2130 (2009). doi:10.1088/0004-637X

M. Amenomori, S. Ayabe, X.J. Bi, D. Chen, S.W. Cui, Danzengluobu, L.K. Ding, X.H. Ding, C.F. Feng, Z. Feng, Z.Y. Feng, X.Y. Gao, Q.X. Geng, H.W. Guo, H.H. He, M. He, K. Hibino, N. Hotta, H. Hu, H.B. Hu, J. Huang, Q. Huang, H.Y. Jia, F. Kajino, K. Kasahara, Y. Katayose, C. Kato, K. Kawata, Labaciren, G.M. Le, A.F. Li, J.Y. Li, Y.-Q. Lou, H. Lu, S.L. Lu, X.R. Meng, K. Mizutani, J. Mu, K. Munakata, A. Nagai, H. Nanjo, M. Nishizawa, M. Ohnishi, I. Ohta, H. Onuma, T. Ouchi, S. Ozawa, J.R. Ren, T. Saito, T.Y. Saito, M. Sakata, T.K. Sako, T. Sasaki, M. Shibata, A. Shiomi, T. Shirai, H. Sugimoto, M. Takita, Y.H. Tan, N. Tateyama, S. Torii, H. Tsuchiya, S. Udo, B. Wang, H. Wang, X. Wang, Y.G. Wang, H.R. Wu, L. Xue, Y. Yamamoto, C.T. Yan, X.C. Yang, S. Yasue, Z.H. Ye, G.C. Yu, A.F. Yuan, T. Yuda, H.M. Zhang, J.L. Zhang, N.J. Zhang, X.Y. Zhang, Y. Zhang, Y. Zhang, Zhaxisangzhu, X.X. Zhou, Tibet AS $\gamma$ Collaboration, Anisotropy 
and Corotation of Galactic Cosmic Rays. Science 314, 439-443 (2006). doi:10.1126/science.1131702

J.W. Armstrong, R. Woo, Solar wind motion within 30 R solar masses - Spacecraft radio scintillation observations. Astron. Astrophys. 103, 415-421 (1981)

J.W. Armstrong, B.J. Rickett, S.R. Spangler, Electron density power spectrum in the local interstellar medium. Astrophys. J. 443, 209221 (1995). doi:10.1086/175515

A.R. Bell, Turbulent amplification of magnetic field and diffusive shock acceleration of cosmic rays. Mon. Not. R. Astron. Soc. 353, 550-558 (2004). doi:10.1111/j.1365-2966.2004.08097.x

A. Bemporad, Spectroscopic Detection of Turbulence in PostCME Current Sheets. Astrophys. J. 689, 572-584 (2008). doi: $10.1086 / 592377$

A. Beresnyak, Spectral Slope and Kolmogorov Constant of MHD Turbulence. Physical Review Letters 106(7), 075001 (2011). doi:10.1103/PhysRevLett.106.075001

A. Beresnyak, Universal Nonlinear Small-Scale Dynamo. Physical Review Letters 108(3), $035002 \quad$ (2012). doi:10.1103/PhysRevLett.108.035002

A. Beresnyak, A. Lazarian, Polarization Intermittency and Its Influence on MHD Turbulence. Astrophys. J. Lett. 640, 175-178 (2006). doi: $10.1086 / 503708$

A. Beresnyak, A. Lazarian, Wave Decay in Magnetohydrodynamic Turbulence. Astrophys. J. 678, 961-967 (2008). doi: $10.1086 / 587052$

A. Beresnyak, A. Lazarian, Comparison of Spectral Slopes of Magnetohydrodynamic and Hydrodynamic Turbulence and Measurements of Alignment Effects. Astrophys. J. 702, 1190-1198 (2009). doi:10.1088/0004-637X

A. Beresnyak, A. Lazarian, Scaling Laws and Diffuse Locality of Balanced and Imbalanced Magnetohydrodynamic Turbulence. Astrophys. J. Lett. 722, 110-113 (2010). doi:10.1088/2041-8205

A. Beresnyak, T.W. Jones, A. Lazarian, Turbulence-Induced Magnetic Fields and Structure of Cosmic Ray Modified Shocks. Astrophys. J. 707, 1541-1549 (2009). doi:10.1088/0004-637X

A. Beresnyak, A. Lazarian, J. Cho, Density Scaling and Anisotropy in Supersonic Magnetohydrodynamic Turbulence. Astrophys. J. Lett. 624, 93-96 (2005). doi:10.1086/430702

A. Beresnyak, H. Yan, A. Lazarian, Numerical Study of Cosmic Ray Diffusion in Magnetohydrodynamic Turbulence. Astrophys. J. 728, 60 (2011). doi:10.1088/0004-637X

L. Bettarini, G. Lapenta, Spontaneous non-steady magnetic reconnection within the solar environment. Astron. Astrophys. 518, 57 (2010). doi:10.1051/0004-6361

A. Bhattacharjee, Y.-M. Huang, H. Yang, B. Rogers, Fast reconnection in high-Lundquist-number plasmas due to the plasmoid Instability. Physics of Plasmas 16(11), 112102 (2009). doi: $10.1063 / 1.3264103$

N.H. Bian, P.K. Browning, Particle Acceleration in a Model of a Turbulent Reconnecting Plasma: A Fractional Diffusion Approach. Astrophys. J. Lett. 687, 111-114 (2008). doi:10.1086/593145

J.W. Bieber, C.W. Smith, W.H. Matthaeus, Cosmic-ray pitch-angle scattering in isotropic turbulence. Astrophys. J. 334, 470-475 (1988). doi:10.1086/166851

J.W. Bieber, W.H. Matthaeus, C.W. Smith, W. Wanner, M.-B. Kallenrode, G. Wibberenz, Proton and electron mean free paths: The Palmer consensus revisited. Astrophys. J. 420, 294-306 (1994). doi:10.1086/173559

D. Biskamp, Magnetic Reconnection in Plasmas. Astrophys. Space Sc. 242, 165-207 (1996). doi:10.1007/BF00645113

D. Biskamp, Magnetohydrodynamic Turbulence 2003

P. Blasi, E. Amato, A Kinetic Approach to Non Resonant Modes and Growth Rates of Streaming Instability: Consequences for Shock Acceleration, in International Cosmic Ray Conference. International Cosmic Ray Conference, vol. 2, 2008, pp. 235-238
S. Boldyrev, On the Spectrum of Magnetohydrodynamic Turbulence. Astrophys. J. Lett. 626, 37-40 (2005). doi:10.1086/431649

S. Boldyrev, Spectrum of Magnetohydrodynamic Turbulence. Physical Review Letters 96(11), 115002 (2006). doi:10.1103/PhysRevLett.96.115002

G. Brunetti, Particle Acceleration Processes in Galaxy Clusters and Future Low Frequency Radio Observations, in IAU Joint Discussion. IAU Joint Discussion, vol. 12, 2006

G. Brunetti, A. Lazarian, Compressible turbulence in galaxy clusters: physics and stochastic particle re-acceleration. Mon. Not. R. Astron. Soc. 378, 245-275 (2007). doi:10.1111/j.13652966.2007.11771.x

G. Brunetti, R. Cassano, K. Dolag, G. Setti, On the evolution of giant radio halos and their connection with cluster mergers. Astron. Astrophys. 507, 661-669 (2009). doi:10.1051/0004-6361

R. Bruno, V. Carbone, The Solar Wind as a Turbulence Laboratory. Living Reviews in Solar Physics 2, 4 (2005)

R. Cassano, G. Brunetti, Cluster mergers and non-thermal phenomena: a statistical magneto-turbulent model. Mon. Not. R. Astron. Soc. 357, 1313-1329 (2005). doi:10.1111/j.1365-2966.2005.08747.x

B.D.G. Chandran, Scattering of Energetic Particles by Anisotropic Magnetohydrodynamic Turbulence with a Goldreich-Sridhar Power Spectrum. Physical Review Letters 85, 4656-4659 (2000). doi:10.1103/PhysRevLett.85.4656

B.D.G. Chandran, Weak Compressible Magnetohydrodynamic Turbulence in the Solar Corona. Physical Review Letters 95(26), 265004 (2005). doi:10.1103/PhysRevLett.95.265004

B.D.G. Chandran, Strong Anisotropic MHD Turbulence with Cross Helicity. Astrophys. J. 685, 646-658 (2008). doi:10.1086/589432

A. Chepurnov, A. Lazarian, Turbulence Spectra from DopplerBroadened Spectral Lines: Tests of the Velocity Channel Analysis and Velocity Coordinate Spectrum Techniques. Astrophys. J. 693, 1074-1083 (2009). doi:10.1088/0004-637X

A. Chepurnov, A. Lazarian, Extending the Big Power Law in the Sky with Turbulence Spectra from Wisconsin $\mathrm{H} \alpha$ Mapper Data. Astrophys. J. 710, 853-858 (2010). doi:10.1088/0004-637X

A. Chepurnov, A. Lazarian, S. Stanimirović, C. Heiles, J.E.G. Peek, Velocity Spectrum for H I at High Latitudes. Astrophys. J. 714, 1398-1406 (2010). doi:10.1088/0004-637X

J. Cho, A. Lazarian, Compressible Sub-Alfvénic MHD Turbulence in Low- $\beta$ Plasmas. Physical Review Letters 88(24), 245001 (2002). doi:10.1103/PhysRevLett.88.245001

J. Cho, A. Lazarian, Compressible magnetohydrodynamic turbulence: mode coupling, scaling relations, anisotropy, viscosity-damped regime and astrophysical implications. Mon. Not. R. Astron. Soc. 345, 325-339 (2003). doi:10.1046/j.1365-8711.2003.06941.x

J. Cho, A. Lazarian, Generation of compressible modes in MHD turbulence. Theoretical and Computational Fluid Dynamics 19, 127157 (2005). doi:10.1007/s00162-004-0157-x

J. Cho, E.T. Vishniac, The Anisotropy of Magnetohydrodynamic Alfvénic Turbulence. Astrophys. J. 539, 273-282 (2000). doi: $10.1086 / 309213$

J. Cho, A. Lazarian, E.T. Vishniac, Simulations of Magnetohydrodynamic Turbulence in a Strongly Magnetized Medium. Astrophys. J. 564, 291-301 (2002). doi:10.1086/324186

J. Cho, A. Lazarian, E.T. Vishniac, MHD Turbulence: Scaling Laws and Astrophysical Implications, in Turbulence and Magnetic Fields in Astrophysics, ed. by E. Falgarone \& T. Passot Lecture Notes in Physics, Berlin Springer Verlag, vol. 614, 2003, pp. 5698

J. Cho, E.T. Vishniac, A. Beresnyak, A. Lazarian, D. Ryu, Growth of Magnetic Fields Induced by Turbulent Motions. Astrophys. J. 693, 1449-1461 (2009). doi:10.1088/0004-637X

A. Ciaravella, J.C. Raymond, The Current Sheet Associated with the 2003 November 4 Coronal Mass Ejection: Density, Temperature, Thickness, and Line Width. Astrophys. J. 686, 1372-1382 (2008). 
doi: $10.1086 / 590655$

J.P.J. Coleman, Turbulence, Viscosity, and Dissipation in the SolarWind Plasma. Astrophys. J. 153, 371 (1968). doi:10.1086/149674

S.R. Cranmer, A.A. van Ballegooijen, On the Generation, Propagation, and Reflection of Alfvén Waves from the Solar Photosphere to the Distant Heliosphere. Astrophys. J. Suppl. S. 156, 265-293 (2005). doi:10.1086/426507

A.C. Cummings, E.C. Stone, Anomalous Cosmic Rays and Solar Modulation. Space Sc. Rev. 83, 51-62 (1998)

W. Daughton, J. Scudder, H. Karimabadi, Fully kinetic simulations of undriven magnetic reconnection with open boundary conditions. Physics of Plasmas 13(7), 072101 (2006). doi:10.1063/1.2218817

W. Daughton, V. Roytershteyn, B.J. Albright, K. Bowers, L. Yin, H. Karimabadi, Reconnection Dynamics in Semi-Collisional Plasmas. AGU Fall Meeting Abstracts, 1705 (2008)

E.M. de Gouveia Dal Pino, A. Lazarian, Ultra-High-Energy CosmicRay Acceleration by Magnetic Reconnection in Newborn Accretion-induced Collapse Pulsars. Astrophys. J. Lett. 536, 3134 (2000). doi:10.1086/312730

E.M. de Gouveia Dal Pino, A. Lazarian, Constraints on the Acceleration of Ultra-High-Energy Cosmic Rays in Accretioninduced Collapse Pulsars. Astrophys. J. 560, 358-364 (2001). doi: $10.1086 / 322509$

E.M. de Gouveia Dal Pino, A. Lazarian, The role of Violent Magnetic Reconnection on the Production of the Large Scale Superluminal Ejections of the Microquasar GRS 1915+105. ArXiv Astrophysics e-prints (2003)

E.M. de Gouveia Dal Pino, A. Lazarian, Production of the large scale superluminal ejections of the microquasar GRS $1915+105$ by violent magnetic reconnection. Astron. Astrophys. 441, 845-853 (2005). doi:10.1051/0004-6361:20042590

E.M. de Gouveia Dal Pino, P.P. Piovezan, L.H.S. Kadowaki, The role of magnetic reconnection on jet/accretion disk systems. Astron. Astrophys. 518, 5 (2010a). doi:10.1051/0004-6361

E.M. de Gouveia Dal Pino, G. Kowal, L.H.S. Kadowaki, P. Piovezan, A. Lazarian, Magnetic Field Effects Near the Launching Region of Astrophysical Jets. International Journal of Modern Physics D 19, 729-739 (2010b). doi:10.1142/S0218271810016920

E.M. de Gouveia Dal Pino, G. Kowal, A. Lazarian, R. Santos-Lima, Particle Acceleration by Magnetic Reconnection in AGNs and in the IGM. ArXiv e-prints (2011)

M.V. del Valle, G.E. Romero, P.L. Luque-Escamilla, J. Martí, J. Ramón Sánchez-Sutil, Are T Tauri Stars Gamma-Ray Emitters? Astrophys. J. 738, 115 (2011). doi:10.1088/0004-637X

P. Desiati, A. Lazarian, Anisotropy of TeV Cosmic Rays and the Outer Heliospheric Boundaries. ArXiv e-prints (2011)

P.H. Diamond, M.A. Malkov, Dynamics of Mesoscale Magnetic Field in Diffusive Shock Acceleration. Astrophys. J. 654, 252-266 (2007). doi:10.1086/508857

N.H. Dieter, W.J. Welch, J.D. Romney, A very small interstellar neutral hydrogen cloud observed with VLBI techniques. Astrophys. J. Lett. 206, 113-115 (1976). doi:10.1086/182145

J. Ding, F. Yuan, E. Liang, Electron Heating and Acceleration by Magnetic Reconnection in Hot Accretion Flows. Astrophys. J. 708, 1545-1550 (2010). doi:10.1088/0004-637X

P. Dmitruk, W.H. Matthaeus, L.J. Milano, S. Oughton, Conditions for sustainment of magnetohydrodynamic turbulence driven by Alfvén waves. Physics of Plasmas 8, 2377-2384 (2001). doi:10.1063/1.1344563

M. Dobrowolny, A. Mangeney, P. Veltri, Properties of magnetohydrodynamic turbulence in the solar wind. Astron. Astrophys. 83, 2632 (1980)

E.A. Dorfi, L.O. Drury, A Cosmic Ray Driven Instability, in International Cosmic Ray Conference, ed. by F. C. Jones International Cosmic Ray Conference, vol. 3, 1985, pp. 121-123

B.T. Draine, A. Lazarian, Electric Dipole Radiation from Spin- ning Dust Grains. Astrophys. J. 508, 157-179 (1998) doi: $10.1086 / 306387$

J.F. Drake, Magnetic explosions in space. Nature 410, 525-526 (2001)

J.F. Drake, M. Swisdak, K.M. Schoeffler, B.N. Rogers, S Kobayashi, Formation of secondary islands during magnetic reconnection. Geophys. Res. Lett. 33, 13105 (2006). doi:10.1029/2006GL025957

J.F. Drake, P.A. Cassak, M.A. Shay, M. Swisdak, E. Quataert, A Magnetic Reconnection Mechanism for Ion Acceleration and Abundance Enhancements in Impulsive Flares. Astrophys. J. Lett. 700, 16-20 (2009). doi:10.1088/0004-637X

J.F. Drake, M. Opher, M. Swisdak, J.N. Chamoun, A Magnetic Reconnection Mechanism for the Generation of Anomalous Cosmic Rays. Astrophys. J. 709, 963-974 (2010). doi:10.1088/0004$637 \mathrm{X}$

L.O. Drury, Reaction effects in diffusive shock acceleration. Advances in Space Research 4, 185-191 (1984). doi:10.1016/02731177(84)90311-9

L.O. Drury, First-order Fermi acceleration driven by magnetic reconnection. Mon. Not. R. Astron. Soc., 2661 (2012). doi:10.1111/j.1365-2966.2012.20804.x

T.H. Dupree, A Perturbation Theory for Strong Plasma Turbulence. Physics of Fluids 9, 1773-1782 (1966). doi:10.1063/1.1761932

B.G. Elmegreen, J. Scalo, Interstellar Turbulence I: Observations and Processes. Annu. Rev. Astron. Astr. 42, 211-273 (2004). doi:10.1146/annurev.astro.41.011802.094859

T.A. Enßlin, C. Vogt, Magnetic turbulence in cool cores of galaxy clusters. Astron. Astrophys. 453, 447-458 (2006). doi:10.1051/00046361:20053518

G.L. Eyink, A. Lazarian, E.T. Vishniac, Fast Magnetic Reconnection and Spontaneous Stochasticity. Astrophys. J. 743, 51 (2011) doi:10.1088/0004-637X

H. Falcke, E. Körding, S. Markoff, A scheme to unify low-power accreting black holes. Jet-dominated accretion flows and the radio/X-ray correlation. Astron. Astrophys. 414, 895-903 (2004). doi:10.1051/0004-6361:20031683

A.J. Farmer, P. Goldreich, Wave Damping by Magnetohydrodynamic Turbulence and Its Effect on Cosmic-Ray Propagation in the Interstellar Medium. Astrophys. J. 604, 671-674 (2004). doi:10.1086/382040

G.M. Felice, R.M. Kulsrud, Cosmic-Ray Pitch-Angle Scattering through 90deg. Astrophys. J. 553, 198-210 (2001). doi: $10.1086 / 320651$

S.E.S. Ferreira, M.S. Potgieter, K. Scherer, Transport and acceleration of anomalous cosmic rays in the inner heliosheath. Journal of Geophysical Research (Space Physics) 112, 11101 (2007). doi:10.1029/2007JA012477

K.M. Ferrière, The interstellar environment of our galaxy. Reviews of Modern Physics 73, 1031-1066 (2001). doi:10.1103/RevModPhys.73.1031

L.A. Fisk, G. Gloeckler, T.H. Zurbuchen, Acceleration of Low-Energy Ions at the Termination Shock of the Solar Wind. Astrophys. J. 644, 631-637 (2006). doi:10.1086/503535

L.A. Fisk, M.L. Goldstein, A.J. Klimas, G. Sandri, The Fokker-Planck Coefficient for Pitch-Angle Scattering of Cosmic Rays. Astrophys. J. 190, 417-428 (1974). doi:10.1086/152893

R. Fitzpatrick, Plasma Physics (Lulu.com, ???, 2008)

V. Florinski, G.P. Zank, Particle acceleration at a dynamic termination shock. Geophys. Res. Lett. 33, 15110 (2006). doi:10.1029/2006GL026371

D.B. Fox, D.A. Frail, P.A. Price, S.R. Kulkarni, E. Berger, T. Piran, A.M. Soderberg, S.B. Cenko, P.B. Cameron, A. Gal-Yam, M.M Kasliwal, D.-S. Moon, F.A. Harrison, E. Nakar, B.P. Schmidt, B. Penprase, R.A. Chevalier, P. Kumar, K. Roth, D. Watson, B.L. Lee, S. Shectman, M.M. Phillips, M. Roth, P.J. McCarthy, M. Rauch, L. Cowie, B.A. Peterson, J. Rich, N. Kawai, K. Aoki, G. Kosugi, T. 
Totani, H.-S. Park, A. MacFadyen, K.C. Hurley, The afterglow of GRB 050709 and the nature of the short-hard $\gamma$-ray bursts. Nature 437, 845-850 (2005). doi:10.1038/nature04189

T.J. Galama, P.M. Vreeswijk, J. van Paradijs, C. Kouveliotou, T. Augusteijn, H. Böhnhardt, J.P. Brewer, V. Doublier, J.-F. Gonzalez, B. Leibundgut, C. Lidman, O.R. Hainaut, F. Patat, J. Heise, J. in't Zand, K. Hurley, P.J. Groot, R.G. Strom, P.A. Mazzali, K. Iwamoto, K. Nomoto, H. Umeda, T. Nakamura, T.R. Young, T. Suzuki, T. Shigeyama, T. Koshut, M. Kippen, C. Robinson, P. de Wildt, R.A.M.J. Wijers, N. Tanvir, J. Greiner, E. Pian, E. Palazzi, F. Frontera, N. Masetti, L. Nicastro, M. Feroci, E. Costa, L. Piro, B.A. Peterson, C. Tinney, B. Boyle, R. Cannon, R. Stathakis, E. Sadler, M.C. Begam, P. Ianna, An unusual supernova in the error box of the $\gamma$-ray burst of 25 April 1998. Nature 395, 670-672 (1998). doi:10.1038/27150

S. Galtier, S.V. Nazarenko, A.C. Newell, A. Pouquet, A weak turbulence theory for incompressible magnetohydrodynamics. Journal of Plasma Physics 63, 447-488 (2000). doi:10.1017/S0022377899008284

S. Galtier, S.V. Nazarenko, A.C. Newell, A. Pouquet, Anisotropic Turbulence of Shear-Alfvén Waves. Astrophys. J. Lett. 564, 49-52 (2002). doi:10.1086/338791

C.W. Gardiner, Handbook of Stochastic Methods for Physics, Chemistry and the Natural Sciences 1994

S. Ghosh, D.J. Thomson, W.H. Matthaeus, L.J. Lanzerotti, Turbulence in the Interplanetary Medium: Can Discrete Modes Co-exist With Turbulence?, in American Institute of Physics Conference Series, ed. by D. Vassiliadis, S.F. Fung, X. Shao, I.A. Daglis, J.D. Huba American Institute of Physics Conference Series, vol. 1320, 2011, pp. 166-173. doi:10.1063/1.3544321

J. Giacalone, J.R. Jokipii, The Transport of Cosmic Rays across a Turbulent Magnetic Field. Astrophys. J. 520, 204-214 (1999). doi: $10.1086 / 307452$

J. Giacalone, J.R. Jokipii, Magnetic Field Amplification by Shocks in Turbulent Fluids. Astrophys. J. Lett. 663, 41-44 (2007). doi:10.1086/519994

D. Giannios, UHECRs from magnetic reconnection in relativistic jets. Mon. Not. R. Astron. Soc. 408, 46-50 (2010). doi:10.1111/j.17453933.2010.00925.x

V.L. Ginzburg, S.I. Syrovatskii, The Origin of Cosmic Rays 1964

P. Goldreich, S. Sridhar, Toward a theory of interstellar turbulence. 2: Strong alfvenic turbulence. Astrophys. J. 438, 763-775 (1995). doi: $10.1086 / 175121$

P. Goldreich, S. Sridhar, Folded Fields as the Source of Extreme RadioWave Scattering in the Galactic Center. Astrophys. J. Lett. 640, 159-162 (2006). doi:10.1086/503668

M.L. Goldstein, A nonlinear theory of cosmic-ray pitch-angle diffusion in homogeneous magnetostatic turbulence. Astrophys. J. 204, 900-919 (1976). doi:10.1086/154239

M.L. Goldstein, A.J. Klimas, G. Sandri, Mirroring in the FokkerPlanck coefficient for cosmic-ray pitch-angle scattering in homogeneous magnetic turbulence. Astrophys. J. 195, 787-799 (1975). doi: $10.1086 / 153383$

M. Gordovskyy, P.K. Browning, Particle Acceleration by Magnetic Reconnection in a Twisted Coronal Loop. Astrophys. J. 729, 101 (2011). doi:10.1088/0004-637X

M. Gordovskyy, P.K. Browning, G.E. Vekstein, Particle Acceleration in Fragmenting Periodic Reconnecting Current Sheets in Solar Flares. Astrophys. J. 720, 1603-1611 (2010). doi:10.1088/0004$637 \mathrm{X}$

T. Gotoh, D. Fukayama, T. Nakano, Velocity field statistics in homogeneous steady turbulence obtained using a high-resolution direct numerical simulation. Physics of Fluids 14, 1065-1081 (2002). doi:10.1063/1.1448296

G. Guillian, J. Hosaka, K. Ishihara, J. Kameda, Y. Koshio, A. Minamino, C. Mitsuda, M. Miura, S. Moriyama, M. Nakahata, T.
Namba, Y. Obayashi, H. Ogawa, M. Shiozawa, Y. Suzuki, A. Takeda, Y. Takeuchi, S. Yamada, I. Higuchi, M. Ishitsuka, T. Kajita, K. Kaneyuki, G. Mitsuka, S. Nakayama, H. Nishino, A. Okada, K. Okumura, C. Saji, Y. Takenaga, S. Desai, E. Kearns, J.L. Stone, L.R. Sulak, W. Wang, M. Goldhaber, D. Casper, W. Gajewski, J. Griskevich, W.R. Kropp, D.W. Liu, S. Mine, M.B. Smy, H.W. Sobel, M.R. Vagins, K.S. Ganezer, J. Hill, W.E. Keig, K. Scholberg, C.W. Walter, R.W. Ellsworth, S. Tasaka, A. Kibayashi, J.G. Learned, S. Matsuno, M.D. Messier, Y. Hayato, A.K. Ichikawa, T. Ishida, T. Ishii, T. Iwashita, T. Kobayashi, T. Nakadaira, K. Nakamura, K. Nitta, Y. Oyama, Y. Totsuka, A.T. Suzuki, M. Hasegawa, I. Kato, H. Maesaka, T. Nakaya, K. Nishikawa, H. Sato, S. Yamamoto, M. Yokoyama, T.J. Haines, S. Dazeley, S. Hatakeyama, R. Svoboda, E. Blaufuss, J.A. Goodman, G.W. Sullivan, D. Turcan, A. Habig, Y. Fukuda, Y. Itow, M. Sakuda, M. Yoshida, S.B. Kim, J. Yoo, H. Okazawa, T. Ishizuka, C.K. Jung, T. Kato, K. Kobayashi, M. Malek, C. Mauger, C. McGrew, E. Sharkey, C. Yanagisawa, Y. Gando, T. Hasegawa, K. Inoue, J. Shirai, A. Suzuki, K. Nishijima, H. Ishino, Y. Watanabe, M. Koshiba, D. Kielczewska, H.G. Berns, R. Gran, K.K. Shiraishi, A.L. Stachyra, K. Washburn, R.J. Wilkes, K. Munakata, Observation of the anisotropy of $10 \mathrm{TeV}$ primary cosmic ray nuclei flux with the Super-Kamiokande-I detector. Phys. Rev. D 75(6), 062003 (2007). doi:10.1103/PhysRevD.75.062003

D.L. Hall, K. Munakata, S. Yasue, S. Mori, C. Kato, M. Koyama, S. Akahane, Z. Fujii, K. Fujimoto, J.E. Humble, A.G. Fenton, K.B. Fenton, M.L. Duldig, Gaussian analysis of two hemisphere observations of galactic cosmic ray sidereal anisotropies. J. Geophys. Res. 104, 6737-6750 (1999). doi:10.1029/1998JA900107

C. Heiles, Tiny-Scale Atomic Structure and the Cold Neutral Medium. Astrophys. J. 481, 193 (1997). doi:10.1086/304033

J.C. Higdon, Density fluctuations in the interstellar medium: Evidence for anisotropic magnetogasdynamic turbulence. I - Model and astrophysical sites. Astrophys. J. 285, 109-123 (1984). doi: $10.1086 / 162481$

D.E. Innes, B. Inhester, W.I. Axford, K. Wilhelm, Bi-directional plasma jets produced by magnetic reconnection on the Sun. Nature 386, 811-813 (1997). doi:10.1038/386811a0

V.V. Izmodenov, R. Kallenbach (eds.), The Physics of the Heliospheric Boundaries, in The Physics of the Heliospheric Boundaries 2006

A.R. Jacobson, R.W. Moses, Nonlocal dc electrical conductivity of a Lorentz plasma in a stochastic magnetic field. Phys. Rev. A 29, 3335-3342 (1984). doi:10.1103/PhysRevA.29.3335

J.R. Jokipii, The Rate of Separation of Magnetic Lines of Force in a Random Magnetic Field. Astrophys. J. 183, 1029-1036 (1973). doi: $10.1086 / 152289$

J.R. Jokipii, Energetic Particles Near and Beyond the Solar-wind Termination, in Physics of the Inner Heliosheath, ed. by J. Heerikhuisen, V. Florinski, G. P. Zank, \& N. V. Pogorelov American Institute of Physics Conference Series, vol. 858, 2006, pp. 143-152. doi:10.1063/1.2359319

J.R. Jokipii, J. Giacalone, The Theory of Anomalous Cosmic Rays. Space Sc. Rev. 83, 123-136 (1998)

F.C. Jones, T.J. Birmingham, T.B. Kaiser, Investigation of Resonance Integrals Occurring in Cosmic-Ray Diffusion Theory. Astrophys. J. Lett. 180, 139 (1973). doi:10.1086/181170

F.C. Jones, T.J. Birmingham, T.B. Kaiser, Partially averaged field approach to cosmic ray diffusion. Physics of Fluids 21, 347-360 (1978). doi:10.1063/1.862233

H. Kang, T.W. Jones, D. Ryu, Acoustic instability in cosmic ray mediated shocks. Astrophys. J. 385, 193-204 (1992). doi: $10.1086 / 170927$

A.P. Kazantsev, Enhancement of a Magnetic Field by a Conducting Fluid. Soviet Journal of Experimental and Theoretical Physics 26, 1031 (1968)

R.S. Klessen, P. Hennebelle, Accretion-driven turbulence as universal 
process: galaxies, molecular clouds, and protostellar disks. Astron. Astrophys. 520, 17 (2010). doi:10.1051/0004-6361

A. Kolmogorov, The Local Structure of Turbulence in Incompressible Viscous Fluid for Very Large Reynolds' Numbers. Akademiia Nauk SSSR Doklady 30, 301-305 (1941)

J. Kóta, J.R. Jokipii, Velocity Correlation and the Spatial Diffusion Coefficients of Cosmic Rays: Compound Diffusion. Astrophys. J. 531, 1067-1070 (2000). doi:10.1086/308492

K. Kotera, A.V. Olinto, The Astrophysics of Ultrahigh-Energy Cosmic Rays. Annu. Rev. Astron. Astr. 49, 119-153 (2011). doi:10.1146/annurev-astro-081710-102620

G. Kowal, A. Lazarian, Scaling relations of compressible MHD turbulence. The Astrophysical Journal Letters 666, 69 (2007)

G. Kowal, A. Lazarian, Velocity Field of Compressible Magnetohydrodynamic Turbulence: Wavelet Decomposition and Mode Scalings. The Astrophysical Journal 720, 742 (2010)

G. Kowal, E.M. de Gouveia Dal Pino, A. Lazarian, Magnetohydrodynamic Simulations of Reconnection and Particle Acceleration: Three-dimensional Effects. The Astrophysical Journal 735, 102 (2011)

G. Kowal, E.M. de Gouveia Dal Pino, A. Lazarian, Particle Acceleration in Turbulence and Weakly Stochastic Reconnection. ArXiv e-prints (2012)

G. Kowal, A. Lazarian, A. Beresnyak, Density Fluctuations in MHD Turbulence: Spectra, Intermittency, and Topology. The Astrophysical Journal 658, 423 (2007)

G. Kowal, A. Lazarian, E.T. Vishniac, K. Otmianowska-Mazur, Numerical Tests of Fast Reconnection in Weakly Stochastic Magnetic Fields. Astrophys. J. 700, 63-85 (2009). doi:10.1088/0004-637X

G. Kowal, A. Lazarian, E.T. Vishniac, K. Otmianowska-Mazur, Reconnection Studies Under Different Types of Turbulence Driving. ArXiv e-prints (2012)

H. Koyama, S.-i. Inutsuka, An Origin of Supersonic Motions in Interstellar Clouds. Astrophys. J. Lett. 564, 97-100 (2002). doi: $10.1086 / 338978$

A.G. Kritsuk, M.L. Norman, Thermal Instability-induced Interstellar Turbulence. Astrophys. J. Lett. 569, 127-131 (2002). doi: $10.1086 / 340785$

R.M. Kulsrud, MHD Description of Plasma, in Basic Plasma Physics: Selected Chapters, Handbook of Plasma Physics, Volume 1, ed. by A. A. Galeev \& R. N. Sudan, 1983, p. 1

R.M. Kulsrud, Plasma Physics for Astrophysics 2005

R.M. Kulsrud, S.W. Anderson, The spectrum of random magnetic fields in the mean field dynamo theory of the Galactic magnetic field. Astrophys. J. 396, 606-630 (1992). doi:10.1086/171743

A. Kupiainen, Nondeterministic Dynamics and Turbulent Transport. Annales Henri Poincaré 4, 713-726 (2003). doi:10.1007/s00023003-0957-3

L.D. Landau, E.M. Lifshitz, The Classical Theory of Fields 1975

U.W. Langner, M.S. Potgieter, Possible Explanations of Anomalous Spectra Observed with Voyager 1 Crossing the Solar Wind Termination Shock, in Physics of the Inner Heliosheath, ed. by J. Heerikhuisen, V. Florinski, G. P. Zank, \& N. V. Pogorelov American Institute of Physics Conference Series, vol. 858, 2006, pp. 233-238. doi:10.1063/1.2359333

G. Lapenta, Self-Feeding Turbulent Magnetic Reconnection on Macroscopic Scales. Physical Review Letters 100(23), 235001 (2008). doi:10.1103/PhysRevLett.100.235001

G. Lapenta, A. Lazarian, Achieving fast reconnection in resistive MHD models via turbulent means. Nonlinear Processes in Geophysics 19, 251-263 (2012). doi:10.5194/npg-19-251-2012

R.B. Larson, Turbulence and star formation in molecular clouds. Mon. Not. R. Astron. Soc. 194, 809-826 (1981)

A. Lazarian, Turbulence Statistics from Spectral Line Observations. ArXiv Astrophysics e-prints (2005)

A. Lazarian, Enhancement and Suppression of Heat Transfer by
MHD Turbulence. Astrophys. J. Lett. 645, 25-28 (2006) doi: $10.1086 / 505796$

A. Lazarian, SINS of Viscosity Damped Turbulence, in SINS - Small Ionized and Neutral Structures in the Diffuse Interstellar Medium, ed. by M. Haverkorn \& W. M. Goss Astronomical Society of the Pacific Conference Series, vol. 365, 2007, p. 324

A. Lazarian, Obtaining Spectra of Turbulent Velocity from Observations. Space Sc. Rev. 143, 357-385 (2009). doi:10.1007/s11214008-9460-y

A. Lazarian, Power of Turbulent Reconnection: Star Formation, Acceleration of Cosmic Rays, Heat Transfer, Flares and Gamma Ray Bursts. ArXiv e-prints (2011)

A. Lazarian, G. Brunetti, Turbulence, reconnection and cosmic rays in galaxy clusters . Memorie della Societa Astronomica Italiana 82, $636(2011)$

A. Lazarian, P. Desiati, Magnetic Reconnection as the Cause of Cosmic Ray Excess from the Heliospheric Tail. Astrophys. J. 722, 188196 (2010). doi:10.1088/0004-637X

A. Lazarian, M. Opher, A Model of Acceleration of Anomalous Cosmic Rays by Reconnection in the Heliosheath. Astrophys. J. 703, 8-21 (2009). doi:10.1088/0004-637X

A. Lazarian, D. Pogosyan, Velocity Modification of H I Power Spectrum. Astrophys. J. 537, 720-748 (2000). doi:10.1086/309040

A. Lazarian, D. Pogosyan, Velocity Modification of the Power Spectrum from an Absorbing Medium. Astrophys. J. 616, 943-965 (2004). doi:10.1086/422462

A. Lazarian, D. Pogosyan, Studying Turbulence Using Dopplerbroadened Lines: Velocity Coordinate Spectrum. Astrophys. J. 652, 1348-1365 (2006). doi: $10.1086 / 508012$

A. Lazarian, D. Pogosyan, Studying Velocity Turbulence from Doppler-broadened Absorption Lines: Statistics of Optical Depth Fluctuations. Astrophys. J. 686, 350-362 (2008). doi: $10.1086 / 591238$

A. Lazarian, E.T. Vishniac, Reconnection in a Weakly Stochastic Field. Astrophys. J. 517, 700-718 (1999). doi:10.1086/307233

A. Lazarian, E.T. Vishniac, Model of Reconnection of Weakly Stochastic Magnetic Field and Its Implications, in Revista Mexicana de Astronomia y Astrofisica Conference Series. Revista Mexicana de Astronomia y Astrofisica, vol. 27, vol. 36, 2009, pp. 81-88

A. Lazarian, E.T. Vishniac, J. Cho, Magnetic Field Structure and Stochastic Reconnection in a Partially Ionized Gas. Astrophys. J. 603, 180-197 (2004). doi:10.1086/381383

A. Lazarian, V. Petrosian, H. Yan, J. Cho, Physics of Gamma-Ray Bursts: Turbulence, Energy Transfer and Reconnection. ArXiv Astrophysics e-prints (2003)

R.J. Leamon, W.H. Matthaeus, C.W. Smith, H.K. Wong, Contribution of Cyclotron-resonant Damping to Kinetic Dissipation of Interplanetary Turbulence. Astrophys. J. Lett. 507, 181-184 (1998). doi: $10.1086 / 311698$

Y. Lithwick, P. Goldreich, Compressible Magnetohydrodynamic Turbulence in Interstellar Plasmas. Astrophys. J. 562, 279-296 (2001). doi: $10.1086 / 323470$

Y. Lithwick, P. Goldreich, Imbalanced Weak Magnetohydrodynamic Turbulence. Astrophys. J. 582, 1220-1240 (2003). doi: $10.1086 / 344676$

Y. Lithwick, P. Goldreich, S. Sridhar, Imbalanced Strong MHD Turbulence. Astrophys. J. 655, 269-274 (2007). doi:10.1086/509884

M.S. Longair, High Energy Astrophysics. Vol.1: Particles, Photons and Their Detection 1992

M.S. Longair, The High-energy Radiation of Active Galactic Nuclei, ed. by Munch, G., Mampaso, A., \& Sánchez, F. 1997, p. 218

M.S. Longair, High Energy Astrophysics 2010

N.F. Loureiro, D.A. Uzdensky, A.A. Schekochihin, S.C. Cowley, T.A. Yousef, Turbulent magnetic reconnection in two dimensions. Mon. Not. R. Astron. Soc. 399, 146-150 (2009). doi:10.1111/j.17453933.2009.00742.x 
R.V.E. Lovelace, Dynamo model of double radio sources. Nature 262 , 649-652 (1976). doi:10.1038/262649a0

S.G. Lucek, A.R. Bell, Non-linear amplification of a magnetic field driven by cosmic ray streaming. Mon. Not. R. Astron. Soc. 314, 65-74 (2000). doi:10.1046/j.1365-8711.2000.03363.x

M. Lyutikov, R. Blandford, Gamma Ray Bursts as Electromagnetic Outflows. ArXiv Astrophysics e-prints (2003)

M.-M. Mac Low, The Energy Dissipation Rate of Supersonic, Magnetohydrodynamic Turbulence in Molecular Clouds. Astrophys. J. 524, 169-178 (1999). doi: $10.1086 / 307784$

R.L. Mace, W.H. Matthaeus, J.W. Bieber, Numerical Investigation of Perpendicular Diffusion of Charged Test Particles in Weak Magnetostatic Slab Turbulence. Astrophys. J. 538, 192-202 (2000). doi: $10.1086 / 309093$

J. Maron, P. Goldreich, Simulations of Incompressible Magnetohydrodynamic Turbulence. Astrophys. J. 554, 1175-1196 (2001). doi:10.1086/321413

S. Masuda, T. Kosugi, H. Hara, S. Tsuneta, Y. Ogawara, A loop-top hard X-ray source in a compact solar flare as evidence for magnetic reconnection. Nature 371, 495-497 (1994). doi:10.1038/371495a0

W.H. Matthaeus, M.L. Goldstein, Measurement of the rugged invariants of magnetohydrodynamic turbulence in the solar wind. J. Geophys. Res. 87, 6011-6028 (1982). doi:10.1029/JA087iA08p06011

W.H. Matthaeus, S.L. Lamkin, Rapid magnetic reconnection caused by finite amplitude fluctuations. Physics of Fluids 28, 303-307 (1985). doi:10.1063/1.865147

W.H. Matthaeus, S.L. Lamkin, Turbulent magnetic reconnection. Physics of Fluids 29, 2513-2534 (1986). doi:10.1063/1.866004

W.H. Matthaeus, P. Dmitruk, L.J. Milano, Mini-conference on plasma turbulence in the corona, heliosphere and interstellar medium. Physics of Plasmas 9, 2440-2445 (2002). doi:10.1063/1.1463067

W.H. Matthaeus, M.L. Goldstein, C. Smith, Evaluation of magnetic helicity in homogeneous turbulence. Physical Review Letters 48, 1256-1259 (1982). doi:10.1103/PhysRevLett.48.1256

W.H. Matthaeus, D.C. Montgomery, M.L. Goldstein, Turbulent generation of outward-traveling interplanetary Alfvenic fluctuations. Physical Review Letters 51, 1484-1487 (1983). doi:10.1103/PhysRevLett.51.1484

W.H. Matthaeus, G. Qin, J.W. Bieber, G.P. Zank, Nonlinear Collisionless Perpendicular Diffusion of Charged Particles. Astrophys. J. Lett. 590, 53-56 (2003). doi:10.1086/376613

D.J. McComas, N.A. Schwadron, An explanation of the Voyager paradox: Particle acceleration at a blunt termination shock. Geophys. Res. Lett. 33, 4102 (2006). doi:10.1029/2005GL025437

C.F. McKee, E.C. Ostriker, Theory of Star Formation. Annu. Rev. Astron. Astr. 45, 565-687 (2007). doi: $10.1146 /$ annurev.astro.45.051806.110602

C.F. McKee, J.P. Ostriker, A theory of the interstellar medium - Three components regulated by supernova explosions in an inhomogeneous substrate. Astrophys. J. 218, 148-169 (1977). doi: $10.1086 / 155667$

J.C. McKinney, D.A. Uzdensky, A reconnection switch to trigger gamma-ray burst jet dissipation. Mon. Not. R. Astron. Soc. 419, 573-607 (2012). doi:10.1111/j.1365-2966.2011.19721.x

D.B. Melrose, Acceleration Mechanisms. ArXiv e-prints (2009)

P. Meszaros, M.J. Rees, Poynting Jets from Black Holes and Cosmological Gamma-Ray Bursts. Astrophys. J. Lett. 482, 29 (1997)

R. Metzler, J. Klafter, The random walk's guide to anomalous diffusion: a fractional dynamics approach. Phys. Reports 339, 1-77 (2000). doi:10.1016/S0370-1573(00)00070-3

H.K. Moffatt, Magnetic Field Generation in Electrically Conducting Fluids 1978

D. Montgomery, L. Turner, Anisotropic magnetohydrodynamic turbulence in a strong external magnetic field. Physics of Fluids 24,
825-831 (1981). doi:10.1063/1.863455

D. Montgomery, M.R. Brown, W.H. Matthaeus, Density fluctuation spectra in magnetohydrodynamic turbulence. J. Geophys. Res. 92, 282-284 (1987). doi:10.1029/JA092iA01p00282

H. Moraal, R.A. Caballero-Lopez, K.G. McCracken, F.B. McDonald, R.A. Mewaldt, V. Ptuskin, M.E. Wiedenbeck, Cosmic Ray Energy Changes at the Termination Shock and in the Heliosheath, in Physics of the Inner Heliosheath, ed. by J. Heerikhuisen, V. Florinski, G. P. Zank, \& N. V. Pogorelov American Institute of Physics Conference Series, vol. 858, 2006, pp. 219-225. doi:10.1063/1.2359331

K. Nagashima, K. Fujimoto, R.M. Jacklyn, Galactic and heliotail-in anisotropies of cosmic rays as the origin of sidereal daily variation in the energy region ;formula $i ; 104 \mathrm{GeV}$. J. Geophys. Res. 103, 17429-17440 (1998). doi:10.1029/98JA01105

F. Nakamura, Z.-Y. Li, Protostellar Turbulence Driven by Collimated Outflows. Astrophys. J. 662, 395-412 (2007). doi: $10.1086 / 517515$

F. Nakamura, C.F. McKee, R.I. Klein, R.T. Fisher, On the Hydrodynamic Interaction of Shock Waves with Interstellar Clouds. II. The Effect of Smooth Cloud Boundaries on Cloud Destruction and Cloud Turbulence. Astrophys. J. Suppl. S. 164, 477-505 (2006). doi: $10.1086 / 501530$

R. Narayan, M.V. Medvedev, Thermal Conduction in Clusters of Galaxies. Astrophys. J. Lett. 562, 129-132 (2001). doi: $10.1086 / 338325$

C.S. Ng, A. Bhattacharjee, Interaction of Shear-Alfven Wave Packets: Implication for Weak Magnetohydrodynamic Turbulence in Astrophysical Plasmas. Astrophys. J. 465, 845 (1996). doi: $10.1086 / 177468$

C.S. Ng, A. Bhattacharjee, Scaling of anisotropic spectra due to the weak interaction of shear-Alfvén wave packets. Physics of Plasmas 4, 605-610 (1997). doi:10.1063/1.872158

N. Nishizuka, K. Shibata, Fractal Reconnection and Particle Acceleration in the Solar Atmosphere, in 38th COSPAR Scientific Assembly, vol. 38, 2010, p. 1959

C.A. Norman, A. Ferrara, The Turbulent Interstellar Medium: Generalizing to a Scale-dependent Phase Continuum. Astrophys. J. 467, 280 (1996). doi: $10.1086 / 177603$

M. Ossendrijver, The solar dynamo. Astron. Astrophys. Rev. 11, 287367 (2003). doi:10.1007/s00159-003-0019-3

E.C. Ostriker, Developing Diagnostics of Molecular Clouds Using Numerical Mhd Simulations, in Turbulence and Magnetic Fields in Astrophysics, ed. by E. Falgarone \& T. Passot Lecture Notes in Physics, Berlin Springer Verlag, vol. 614, 2003, pp. 252-270

M. Ostrowski, Mechanisms and sites of ultra high energy cosmic ray origin. Astroparticle Physics 18, 229-236 (2002). doi:10.1016/S0927-6505(02)00154-8

S. Oughton, P. Dmitruk, W.H. Matthaeus, Coronal Heating and Reduced Mhd, in Turbulence and Magnetic Fields in Astrophysics, ed. by E. Falgarone \& T. Passot Lecture Notes in Physics, Berlin Springer Verlag, vol. 614, 2003, pp. 28-55

A.J. Owens, The Effects of Nonlinear Terms in Cosmic-Ray Diffusion Theory. Astrophys. J. 191, 235-244 (1974). doi:10.1086/152960

B. Paczynski, Gamma-ray bursters at cosmological distances. Astrophys. J. Lett. 308, $43-46$ (1986). doi:10.1086/184740

P. Padoan, R. Jimenez, M. Juvela, Å. Nordlund, The Average Magnetic Field Strength in Molecular Clouds: New Evidence of Super-Alfvénic Turbulence. Astrophys. J. Lett. 604, 49-52 (2004). doi: $10.1086 / 383308$

P. Padoan, M. Juvela, A. Kritsuk, M.L. Norman, The Power Spectrum of Turbulence in NGC 1333: Outflows or Large-Scale Driving? Astrophys. J. Lett. 707, 153-157 (2009). doi:10.1088/0004-637X

E.N. Parker, Sweet's Mechanism for Merging Magnetic Fields in Conducting Fluids. J. Geophys. Res. 62, 509-520 (1957). doi:10.1029/JZ062i004p00509 
E.N. Parker, Dynamics of the Interplanetary Gas and Magnetic Fields. Astrophys. J. 128, 664 (1958). doi:10.1086/146579

E.N. Parker, The Generation of Magnetic Fields in Astrophysical Bodies. I. The Dynamo Equations. Astrophys. J. 162, 665 (1970). doi: $10.1086 / 150697$

E.N. Parker, Cosmical Magnetic Fields: Their Origin and Their Activity 1979

E.N. Parker, A solar dynamo surface wave at the interface between convection and nonuniform rotation. Astrophys. J. 408, 707-719 (1993). doi:10.1086/172631

T. Passot, E. Vázquez-Semadeni, Density probability distribution in one-dimensional polytropic gas dynamics. Phys. Rev. E 58, 45014510 (1998). doi:10.1103/PhysRevE.58.4501

J.C. Perez, S. Boldyrev, Role of Cross-Helicity in Magnetohydrodynamic Turbulence. Physical Review Letters 102(2), 025003 (2009). doi:10.1103/PhysRevLett.102.025003

V. Petrosian, A. Bykov, Y. Rephaeli, Nonthermal Radiation Mechanisms. Space Sc. Rev. 134, 191-206 (2008). doi:10.1007/s11214008-9327-2

A. Petrosyan, A. Balogh, M.L. Goldstein, J. Léorat, E. Marsch, K. Petrovay, B. Roberts, R. von Steiger, J.C. Vial, Turbulence in the Solar Atmosphere and Solar Wind. Space Sc. Rev. 156, 135-238 (2010). doi:10.1007/s11214-010-9694-3

H.E. Petschek, Magnetic Field Annihilation. NASA Special Publication 50, 425 (1964)

T. Piran, Gamma-ray bursts and the fireball model. Phys. Reports 314, 575-667 (1999). doi:10.1016/S0370-1573(98)00127-6

T. Piran, Magnetic Fields in Gamma-ray Bursts: A Short Overview, in Magnetic Fields in the Universe: From Laboratory and Stars to Primordial Structures., ed. by E. M. de Gouveia dal Pino, G. Lugones, \& A. Lazarian American Institute of Physics Conference Series, vol. 784, 2005, pp. 164-174. doi:10.1063/1.2077181

N.V. Pogorelov, J. Heerikhuisen, G.P. Zank, J.J. Mitchell, I.H. Cairns, Heliospheric asymmetries due to the action of the interstellar magnetic field. Advances in Space Research 44, 1337-1344 (2009a). doi:10.1016/j.asr.2009.07.019

N.V. Pogorelov, S.N. Borovikov, G.P. Zank, T. Ogino, ThreeDimensional Features of the Outer Heliosphere Due to Coupling Between the Interstellar and Interplanetary Magnetic Fields. III. The Effects of Solar Rotation and Activity Cycle. Astrophys. J. 696, 1478-1490 (2009b). doi:10.1088/0004-637X

E.R. Priest, T.G. Forbes, The magnetic nature of solar flares. Astron. Astrophys. Rev. 10, 313-377 (2002). doi: $10.1007 / \mathrm{s} 001590100013$

G. Qin, W.H. Matthaeus, J.W. Bieber, Perpendicular Transport of Charged Particles in Composite Model Turbulence: Recovery of Diffusion. Astrophys. J. Lett. 578, 117-120 (2002). doi: $10.1086 / 344687$

M.A. Riquelme, A. Spitkovsky, Nonlinear Study of Bell's Cosmic Ray Current-Driven Instability. Astrophys. J. 694, 626-642 (2009). doi: $10.1088 / 0004-637 X$

D.A. Roberts, M.L. Goldstein, L.W. Klein, W.H. Matthaeus, Origin and evolution of fluctuations in the solar wind - HELIOS observations and Helios-Voyager comparisons. J. Geophys. Res. 92, 12023-12035 (1987a). doi:10.1029/JA092iA11p12023

D.A. Roberts, L.W. Klein, M.L. Goldstein, W.H. Matthaeus, The nature and evolution of magnetohydrodynamic fluctuations in the solar wind - Voyager observations. J. Geophys. Res. 92, 1102111040 (1987b). doi:10.1029/JA092iA10p11021

F. Sahraoui, M. Goldstein, Structures and Intermittency in Small Scales Solar Wind Turbulence. Twelfth International Solar Wind Conference 1216, 140-143 (2010). doi:10.1063/1.3395820

K.H. Schatten, Large-scale properties of the interplanetary magnetic field. Reviews of Geophysics and Space Physics 9, 773-812 (1971)

A.A. Schekochihin, S.C. Cowley, W. Dorland, Interplanetary and inter- stellar plasma turbulence. Plasma Physics and Controlled Fusion 49, 195 (2007). doi:10.1088/0741-3335

A.A. Schekochihin, S.C. Cowley, J.L. Maron, J.C. McWilliams, Critical Magnetic Prandtl Number for Small-Scale Dynamo. Physical Review Letters 92(5), 054502 (2004). doi:10.1103/PhysRevLett.92.054502

A.A. Schekochihin, S.C. Cowley, W. Dorland, G.W. Hammett, G.G. Howes, E. Quataert, T. Tatsuno, Astrophysical Gyrokinetics: Kinetic and Fluid Turbulent Cascades in Magnetized Weakly Collisional Plasmas. Astrophys. J. Suppl. S. 182, 310-377 (2009). doi:10.1088/0067-0049

R. Schlickeiser, Particle Acceleration Processes in Cosmic Plasmas, in Energy Conversion and Particle Acceleration in the Solar Corona, ed. by L. Klein Lecture Notes in Physics, Berlin Springer Verlag, vol. 612, 2003, pp. 230-260

J.A. Sellwood, S.A. Balbus, Differential Rotation and Turbulence in Extended H I Disks. Astrophys. J. 511, 660-665 (1999). doi: $10.1086 / 306728$

M.A. Shay, J.F. Drake, R.E. Denton, D. Biskamp, Structure of the dissipation region during collisionless magnetic reconnection. J. Geophys. Res. 103, 9165-9176 (1998). doi:10.1029/97JA03528

J.V. Shebalin, W.H. Matthaeus, D. Montgomery, Anisotropy in MHD turbulence due to a mean magnetic field. Journal of Plasma Physics 29, 525-547 (1983). doi:10.1017/S0022377800000933

A. Shemi, T. Piran, The appearance of cosmic fireballs. Astrophys. J. Lett. 365, 55-58 (1990). doi:10.1086/185887

K. Shibata, S. Tanuma, Plasmoid-induced-reconnection and fractal reconnection. Earth, Planets, and Space 53, 473-482 (2001)

T. Shimizu, K. Kondoh, M. Ugai, Three-dimensional non-linear instability of spontaneous fast magnetic reconnection. Earth, Planets, and Space 61, 569-572 (2009a)

T. Shimizu, K. Kondoh, K. Shibata, M. Ugai, Magnetohydrodynamic study of three-dimensional instability of the spontaneous fast magnetic reconnection. Physics of Plasmas 16(5), 052903 (2009b). doi:10.1063/1.3095562

J. Skilling, Cosmic ray streaming. I - Effect of Alfven waves on particles. Mon. Not. R. Astron. Soc. 172, 557-566 (1975)

T.W. Speiser, Conductivity without collisions or noise. Planet. Space Sc. 18, 613 (1970). doi:10.1016/0032-0633(70)90136-4

S. Stanimirović, J.M. Weisberg, A. Hedden, K. Devine, T. Green, S.B. Anderson, The Tiny-Scale Atomic Structure: Gas Cloudlets or Scintillation Phenomenon? Astrophys. Space Sc. 292, 103-109 (2004). doi:10.1023/B:ASTR.0000045005.36554.36

M. Stix, Modulation of Acoustic Waves by Solar Convection. Sol. Phys. 196, 19-27 (2000)

J.M. Stone, E.C. Ostriker, C.F. Gammie, Dissipation in Compressible Magnetohydrodynamic Turbulence. Astrophys. J. Lett. 508, 99102 (1998). doi: $10.1086 / 311718$

H.R. Strauss, Nonlinear, three-dimensional magnetohydrodynamics of noncircular tokamaks. Physics of Fluids 19, 134-140 (1976). doi: $10.1063 / 1.861310$

M. Strauss, Buildup of X-ray laser gain by fluctuations in channeled relativistic beam systems. Phys. Rev. A 38, 1358-1362 (1988). doi:10.1103/PhysRevA.38.1358

P.A. Sturrock, Model of the High-Energy Phase of Solar Flares. Nature 211, 695-697 (1966). doi:10.1038/211695a0

S.T. Suess, S. Nerney, Flow speed inside the brightness boundary of coronal streamers. Geophys. Res. Lett. 33, 10104 (2006). doi:10.1029/2006GL026182

P.A. Sweet, The Neutral Point Theory of Solar Flares, in Electromagnetic Phenomena in Cosmical Physics, ed. by B. Lehnert IAU Symposium, vol. 6, 1958, p. 123

C. Thompson, A Model of Gamma-Ray Bursts. Mon. Not. R. Astron. Soc. 270, 480 (1994)

A.C. Ting, D. Montgomery, W.H. Matthaeus, Turbulent relaxation processes in magnetohydrodynamics. Physics of Fluids 29, 3261- 
3274 (1986). doi:10.1063/1.865843

V.V. Usov, Millisecond pulsars with extremely strong magnetic fields as a cosmological source of gamma-ray bursts. Nature 357, 472474 (1992). doi:10.1038/357472a0

D.A. Uzdensky, Magnetic Reconnection in Extreme Astrophysical Environments. Space Sc. Rev. 160, 45-71 (2011). doi:10.1007/s11214-011-9744-5

D.A. Uzdensky, J.C. McKinney, Magnetic reconnection with radiative cooling. I. Optically thin regime. Physics of Plasmas 18(4), 042105 (2011). doi:10.1063/1.3571602

E. Vázquez-Semadeni, N. García, The Probability Distribution Function of Column Density in Molecular Clouds. Astrophys. J. 557, 727-735 (2001). doi:10.1086/321688

A. Verdini, M. Velli, Alfvén Waves and Turbulence in the Solar Atmosphere and Solar Wind. Astrophys. J. 662, 669-676 (2007). doi: $10.1086 / 510710$

S. Vernetto, Z. Guglielmotto, J.L. Zhang, for the ARGO-YBJ Collaboration, Sky monitoring with ARGO-YBJ. ArXiv e-prints (2009)

J.G. Vestuto, E.C. Ostriker, J.M. Stone, Spectral Properties of Compressible Magnetohydrodynamic Turbulence from Numerical Simulations. Astrophys. J. 590, 858-873 (2003). doi:10.1086/375021

E.T. Vishniac, A. Lazarian, Fast Reconnection in a Weakly Stochastic Field, in Plasma Turbulence and Energetic Particles in Astrophysics, ed. by M. Ostrowski \& R. Schlickeiser, 1999, pp. 182189

E.T. Vishniac, A. Lazarian, J. Cho, Problems and Progress in Astrophysical Dynamos, in Turbulence and Magnetic Fields in Astrophysics, ed. by E. Falgarone \& T. Passot Lecture Notes in Physics, Berlin Springer Verlag, vol. 614, 2003, pp. 376-401

A. Vladimirov, D.C. Ellison, A. Bykov, Nonlinear Diffusive Shock Acceleration with Magnetic Field Amplification. Astrophys. J. 652, 1246-1258 (2006). doi:10.1086/508154

L. Vlahos, H. Isliker, F. Lepreti, Particle Acceleration in an Evolving Network of Unstable Current Sheets. Astrophys. J. 608, 540-553 (2004). doi:10.1086/386364

L. Vlahos, H. Isliker, Y. Kominis, K. Hizanidis, Normal and Anomalous Diffusion: A Tutorial. ArXiv e-prints (2008)

H.J. Völk, Nonlinear Perturbation Theory for Cosmic Ray Propagation in Random Magnetic Fields. Astrophys. Space Sc. 25, 471-490 (1973). doi:10.1007/BF00649186

H.J. Völk, Cosmic ray propagation in interplanetary space. Reviews of Geophysics and Space Physics 13, 547-566 (1975)

F.L. Waelbroeck, Current sheets and nonlinear growth of the $\mathrm{m}=1$ kink-tearing mode. Physics of Fluids B 1, 2372-2380 (1989). doi:10.1063/1.859172

H. Yan, A. Lazarian, Scattering of Cosmic Rays by Magnetohydrodynamic Interstellar Turbulence. Physical Review Letters 89, 1102 (2002). doi:10.1103/PhysRevLett.89.281102

H. Yan, A. Lazarian, Cosmic-Ray Scattering and Streaming in Compressible Magnetohydrodynamic Turbulence. Astrophys. J. 614, 757-769 (2004). doi:10.1086/423733

H. Yan, A. Lazarian, Cosmic-Ray Propagation: Nonlinear Diffusion Parallel and Perpendicular to Mean Magnetic Field. Astrophys. J. 673, 942-953 (2008). doi:10.1086/524771

H. Yan, A. Lazarian, V. Petrosian, Particle Acceleration by Fast Modes in Solar Flares. Astrophys. J. 684, 1461-1468 (2008). doi:10.1086/589962

P.K. Yeung, Y. Zhou, Universality of the Kolmogorov constant in numerical simulations of turbulence. Phys. Rev. E 56, 1746-1752 (1997). doi:10.1103/PhysRevE.56.1746

T. Yokoyama, K. Shibata, Magnetic reconnection as the origin of Xray jets and $\mathrm{H} \alpha$ surges on the Sun. Nature 375, 42-44 (1995). doi: $10.1038 / 375042 \mathrm{a} 0$

G.P. Zank, W.H. Matthaeus, The equations of reduced magnetohydrodynamics. Journal of Plasma Physics 48, 85 (1992a).
doi:10.1017/S002237780001638X

G.P. Zank, W.H. Matthaeus, Waves and turbulence in the solar wind. J. Geophys. Res. 97, 17189 (1992b). doi:10.1029/92JA01734

B. Zhang, H. Yan, The Internal-collision-induced Magnetic Reconnection and Turbulence (ICMART) Model of Gamma-ray Bursts. Astrophys. J. 726, 90 (2011). doi:10.1088/0004-637X

M. Zhang, Acceleration of Galactic and Anomalous Cosmic Rays in the Heliosheath, in Physics of the Inner Heliosheath, ed. by J. Heerikhuisen, V. Florinski, G. P. Zank, \& N. V. Pogorelov American Institute of Physics Conference Series, vol. 858, 2006, pp. 226-232. doi:10.1063/1.2359332

V.V. Zharkova, K. Arzner, A.O. Benz, P. Browning, C. Dauphin, A.G. Emslie, L. Fletcher, E.P. Kontar, G. Mann, M. Onofri, V. Petrosian, R. Turkmani, N. Vilmer, L. Vlahos, Recent Advances in Understanding Particle Acceleration Processes in Solar Flares. Space Sc. Rev. 159, 357-420 (2011). doi:10.1007/s11214-011-9803-y

G. Zimbardo, A. Greco, L. Sorriso-Valvo, S. Perri, Z. Vörös, G. Aburjania, K. Chargazia, O. Alexandrova, Magnetic Turbulence in the Geospace Environment. Space Sc. Rev. 156, 89-134 (2010). doi:10.1007/s11214-010-9692-5

V.N. Zirakashvili, V.S. Ptuskin, H.J. Völk, Modeling Bell's Nonresonant Cosmic-Ray Instability. Astrophys. J. 678, 255-261 (2008). doi:10.1086/529579

E.G. Zweibel, Cosmic-Ray History and Its Implications for Galactic Magnetic Fields. Astrophys. J. 587, 625-637 (2003). doi: $10.1086 / 368256$ 